\title{
MULTIRESOLUTIONAL CHARACTERIZATION AND MITIGATION OF GNSS SIGNAL FOR ROBUST POSITIONING
}

\author{
by \\ Mohammad Hossein Aram \\ B. Eng. Electrical and Computer Engineering \\ Ryerson University, Toronto, 2005
}

A thesis

presented to Ryerson University

in partial fulfillment of the

requirement for the degree of

Master of Applied Science

in the Program of

Electrical and Computer Engineering

Toronto, Ontario, Canada, 2007

(c) Mohammad Hossein Aram 


\section{Author's Declaration}

I hereby declare that I am the sole author of this thesis.

I authorize Ryerson University to lend this thesis to other institutions or individuals for the purpose of scholarly research.

Signature

I further authorize Ryerson University to reproduce this thesis by photocopying or by other means, in total or in part, at the request of other institutions or individuals for the purpose of scholarly research.

Signature 


\section{Instructions on Borrowers}

Ryerson University requires the signatures of all persons using or photocopying this thesis. Please sign below, and give address and date. 
Mohammad Hossein Aram, M. A. Sc., Electrical and Computer Engineering, Ryerson University, Toronto, 2007.

\begin{abstract}
While the use of wavelet filtering on applications such as audio and video is known, in this research. wavelet filters are applied as a practical tool to improve positioning accuracy of a navigation-grade receiver in challenging environments. A single, stationary receiver operating on the L1 frequency, and collecting data in 15-minute segments, was used to obtain pseudoranges which were then used to compute positions. The magnitudes of these pseudoranges are often overstated due to multipath. Multipath mitigation was applied to these signals using a two-stage wavelet filter. The first stage operates in the pseudorange domain to remove bias error and the second stage operates in the position domain to minimize the effect of the low velocities that existed among the stationary positions. This filtering had a marked effect of reducing positioning scatter (variance). To measure the effect of this filtering, several statistical moments (before and after filtering), were compared. Throughout datasets studied, the unfiltered position scatters tend to be markedly non-Gaussian showing extreme effects of skew and kurtosis in addition to high variance. The position scatters after filtering tend to be highly Gaussian with far lower degrees of skew and kurtosis. In this study, the results obtained from the data sets showed significant improvement, less than 1.5 $\mathrm{m}$ with a probability of $96.5 \%$, in standard deviation of the estimated positions.
\end{abstract}




\section{Acknowledgments}

I would like to express my deep appreciation to my advisors, Dr. Sri Krishnan and Dr. Alagan Anpalagan, for their guidance and for providing excellent research opportunities during my studies. Thank you Dr. Krishnan and Dr. Anpalagan.

I would also like to thank Mr. Bern Grush and Mr. Aiden Morrison for their help and support during summer 2005. The author would like to acknowledge Ontario Graduate Scholarship (OGS) program for their financial support as well.

Thank you.

Mohammad Hossein Aram 


\section{Contents}

1 Introduction 1

1.1 Global Positioning System (GPS) Overview . . . . . . . . . . . . . 2

1.2 GPS Architecture . . . . . . . . . . . . . . . . . . . . 3

1.2 .1 Space Segment . . . . . . . . . . . . . . . . . . 3

1.2 .2 Control Segment $\ldots \ldots \ldots \ldots \ldots \ldots$

1.2 .3 User Segment . . . . . . . . . . . . . . . . . . . . . 5

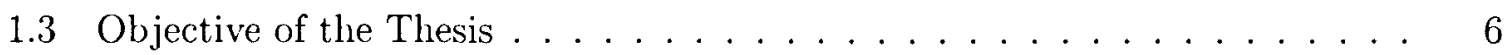

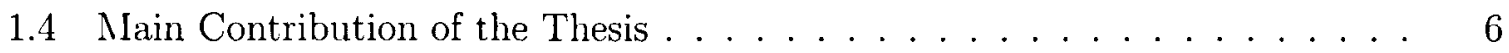

1.5 Organization of the Thesis $\ldots \ldots \ldots \ldots \ldots \ldots$

2 GPS Signal Structure and Characteristics $\quad 8$

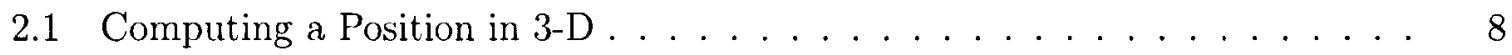

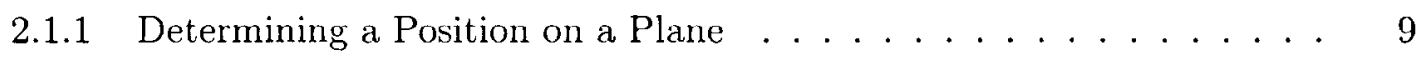

2.1.2 Determining a Position on Three Dimensional Space . . . . . . . 9

2.1 .3 Position Calculations . . . . . . . . . . . . . . . . . 10

2.2 GPS Signal Structure . . . . . . . . . . . . . . . . . . 13

2.2.1 Fundamentals of Spread Spectrum Signaling . . . . . . . . . . . 13

2.3 GPS Signal (C/A Code) Characteristics . . . . . . . . . . . . 14

$2.3 .1 \quad \mathrm{C} / \mathrm{A}$ Code Generation $\ldots \ldots \ldots \ldots \ldots \ldots$

2.3.2 Auto and Cross Correlation of the C/A Codes . . . . . . . . 16

2.3.3 The Transmitted Satellite Signals . . . . . . . . . . . . . . 18

2.4 Error Sources . . . . . . . . . . . . . . . . . . . . . . . . . . . . 19 
2.4 .1 Received Signals . . . . . . . . . . . . . . . 20

2.4 .2 Multipath Error Modeling . . . . . . . . . . . . . . . 21

2.5 Current Multipath Mitigation Methods . . . . . . . . . . . . . . . . 26

2.6 Related Work . . . . . . . . . . . . . . . . . . . . . . . . . 27

3 Wavelet Analysis of GPS Signal $\quad 29$

3.1 Wavelet Analysis Overview . . . . . . . . . . . . . . . . . . . . . . 29

3.1.1 Multilevel Representation of a Function . . . . . . . . . . . . . 33

3.2 Discrete Wavelet Transform $($ DWT $) \ldots \ldots \ldots \ldots \ldots$

3.3 Filter Banks . . . . . . . . . . . . . . . . . . . . . . 36

3.4 De-noising . . . . . . . . . . . . . . . . . . . . . 38

3.4 .1 Noise Reduction Nodel . . . . . . . . . . . . . . . . . . . . . . 39

3.4 .2 Threshold Selection . . . . . . . . . . . . . . . . . . . 11

3.5 Conventional Thresholding . . . . . . . . . . . . . . . . . . 42

3.6 The Proposed Thresholding Method . . . . . . . . . . . . . . . . . . 44

3.6 .1 Modeling the Data . . . . . . . . . . . . . . . . . . 46

3.7 Chapter Summary . . . . . . . . . . . . . . . . . . . . . . 47

4 Multipath Mitigation Strategies $\quad 49$

4.1 Proposed Algorithm . . . . . . . . . . . . . . . . . . . 49

4.1 .1 Introduction . . . . . . . . . . . . . . . . . . 49

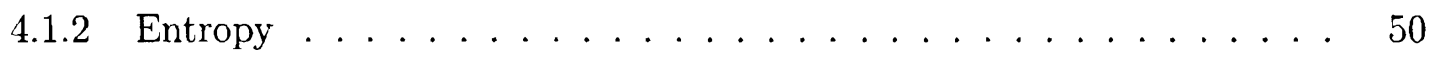

4.1 .3 Norm of the Entropies . . . . . . . . . . . . . . . . 52

4.2 Gencrating Synthetic Signal . . . . . . . . . . . . . . . 54

4.3 Applying the Proposed Algorithm on the Real GPS Data . . . . . . . . . 57

5 Results and Discussions $\quad 59$

5.1 Experimental Setup . . . . . . . . . . . . . . . . . . . . . 59

5.2 Residual (Signal) Modeling . . . . . . . . . . . . . . . 61

5.3 Performance Analysis . . . . . . . . . . . . . . . . . 63 
5.3.1 Wavelet Analysis on Residuals, Thresholding . . . . . . . . . . 63

5.3.2 Wavelet Analysis on Computed Positions . . . . . . . . . . . . . . 66

5.4 Chapter Summary . . . . . . . . . . . . . . . . 74

$\begin{array}{lll}6 & \text { Conclusions } & 76\end{array}$

A

77

Bibliography

78

viii 


\section{List of Figures}

1.1 GPS Satellites Orbiting the Earth,taken from $[6] . \ldots \ldots \ldots 2$

1.2 Detailed GPS Transmitted Signal, C/A on L1 Band. . . . . . . . . . . 4

1.3 Block Diagram of the Proposed Algorithm. . . . . . . . . . . . . 6

2.1 Positioning on 2 Dimensional Plane. . . . . . . . . . . . . . 9

2.2 Positioning on 3 Dimensional Space, taken from $[6] \ldots \ldots \ldots$

$2.3 \mathrm{C} / \mathrm{A}$ Code Generation, taken from $[8] . \ldots \ldots \ldots \ldots$

2.4 The First $1023 \mathrm{C} / \mathrm{A}$ (PRN6) Code. . . . . . . . . . . . . . . 17

2.5 Auto and Cross correlation of the C/A Code (PRN6 and PRN7). . . . . 17

2.6 GPS Transmitted Signal, C/A on L1 Band. . . . . . . . . . . 18

2.7 Generated Multipath Signals. . . . . . . . . . . . . . . . . 23

2.8 The Effect of Rayleigh Channel on the GPS Signals. . . . . . . . . . 25

2.9 Bit Error Rate of Rayleigh Channel. . . . . . . . . . . . . . 25

3.1 A Typical Wavelet $(\mathrm{db} 2) \ldots \ldots \ldots \ldots \ldots$

3.2 Constant Resolution of a Windowed Signal (STFT) . . . . . . . . . . . 30

3.3 The Windowed Fourier Trensform of the Raw Signal Using a Sliding Window. 31

3.4 One Representation of a Time-Frequency Tile for the Wavelet Transform. . . 32

3.5 Scalogram of the Signal. . . . . . . . . . . . . . . . . . . 33

3.6 Daubechies (db2) Scaling and Corresponding Wavelet Functions. . . . . . . 35

3.7 A Typical Block Diagram of Wavelet Analysis. . . . . . . . . . . . . . . 37

3.8 A Three level Wavelet Decomposition. . . . . . . . . . . . . 38

3.9 Illustration of Soft and Hard Thresholding . . . . . . . . . . . . . 39 
3.10 Soft and Hard Thresholding of the real GPS Data . . . . . . . . . . . 40

3.11 A Typical Residual Data to Be Analyzed. . . . . . . . . . . . . . . 43

3.12 Thresholding the Wavelet Coefficients Based on Donoho's Equation. . . . . . 43

3.13 Thresholding the Wavelet Coefficients Based on Donoho's Equation. . . . . . 44

3.14 Wavelet Analysis of a GPS Data. . . . . . . . . . . . . . . . . . . 44

3.15 Soft and Hard Thresholding of the real GPS Data . . . . . . . . . . . . 48

4.1 Entropies Corresponding to Each of the Satellites. . . . . . . . . . . . 51

4.2 Entropies Corresponding to the Whole Data Set. . . . . . . . . . . . 52

4.3 Average of Entropies Corresponding to Each Satellites . . . . . . . . . . 53

4.4 Average of Entropies Corresponding to Each Locations . . . . . . . . . . . 53

4.5 Norm of the Entropies. . . . . . . . . . . . . . . . . . 55

4.6 Normalized Entropy of the Locations. . . . . . . . . . . . . . . . . 55

4.7 Synthetic Multipath Signal. . . . . . . . . . . . . . . . . . . 56

4.8 Normalized RMS Value of the Distortion. . . . . . . . . . . . . . . 57

4.9 Wavelet Analysis of a GPS Data. . . . . . . . . . . . . . 58

4.10 Wavelet Analysis of a GPS Data. . . . . . . . . . . . . . . . 58

5.1 Data Collection Setup. . . . . . . . . . . . . . . . 59

5.2 The Four Locations Where Data Was Collected. . . . . . . . . . . . . 60

5.3 Easting and North Errors of the Second Location, from Right-side of Fig. 5.2. 60

5.4 Typical Code-Carrier Data. . . . . . . . . . . . . . . . 62

5.5 Comparison of the Positions Precision Resulted from Eqs. 3.22 and 3.35. . . 64

5.6 Comparison of the Positions Precision Resulted from Eqs. 3.22 and 3.35, Another Location. . . . . . . . . . . . . . . . . . . . . . . 64

5.7 Improvement and Degradation of the Standard Deviation of 29 Data Sets. . $\quad 66$

5.8 Effect of Leveling on the Scatter Points, level=2 . . . . . . . . . . . . . 67

5.9 Norm and Cumulative Distribution Function (cdf) Improvements. . . . . . 68

5.10 Effect of Leveling on the Scatter Points, level=10 . . . . . . . . . . . . 69 
5.11 Norm of the Standard Deviation Improvements, Level=10. . . . . . . . 69

5.12 Final Norm and Cumulative Distribution Function (cdf) Improvements. . . . 70

5.13 Kurtosis of the Whole Data Sets. . . . . . . . . . . . . . 71

5.14 Skewness of the Whole Data Sets. . . . . . . . . . . . . . . 72

5.15 Actual Computed Positions of a Location. . . . . . . . . . . . . 72

5.16 A Location in Down Town Toronto. . . . . . . . . . . . . . . . . . . 73

5.17 Block Diagram of the Proposed Positioning. . . . . . . . . . . . . 74

A.1 C/A Code Phase Assignment, taken from $[2] . \ldots \ldots \ldots 77$ 


\section{List of Tables}

4.1 Entropies of the Example. . . . . . . . . . . . . . . . . 54

5.1 Comparison of the $S_{\text {norm }}$ for the Location Shown in Fig. 5.6. . . . . . . 65 


\section{Chapter 1}

\section{Introduction}

The Global Positioning system (GPS) is a satellite based system which was initially developed for military purposes to track targets by the U.S. Department of Defense (DoD); but beyond its original uses, the GPS has proved to be an asset in variety of civilian applications as well. Flight, marine, land navigations, logistics, asset location, survey, construction, animal husbandry, and recreation are just a few to name for the GPS applications. One of the GPS usage is to pinpoint a location on the Earth. One's position could be reported with an accuracy up to $300 \mathrm{~m}$, as if the user was in the right place or anywhere on a circle of radius $300 \mathrm{~m}$. Interestingly, this huge difference comes from a tiny time error as low as $1 \mu$ s. Eliminating this tiny error has been of the interest for many years. Estimating a signal that is corrupted by noise has been the focus of many researchers for practical as well as theoretical reasons. The problem is to recover the original signal from the noisy data. We want the recovered signal to be as close as possible to the original signal, retaining most of its important properties (e.g. smoothness). Traditional denoising schemes are based on linear methods, where the most common choice is the Wiener filtering. Recently, nonlinear methods, especially those based on wavelets have become increasingly popular [1]. The application of wavelets in signal processing especially in the area of Global Navigation Satellite System (GNSS) has been of interest for many years. Wavelets are applied to the GNSS signal, i.e. GPS (Global Positioning System), to denoise or mitigate multipath error which in turn helps to decrease the standard deviation of the computed positions. 


\subsection{Global Positioning System (GPS) Overview}

GPS is a line-of-sight, all weather, world-wide continuously available satellite-based Radio Frequency (RF) positioning system, providing three-dimensional position, velocity, and time capability to end-users with an appropriate receiver. The system may be viewed as consisting of space (the actual satellites), control (management of satellite operations), and user segments. The user segment includes all aspects of the equipment required for civil and military use of the system. The full operational constellation of GPS was declared in April 1995 with the baseline GPS system being specified for 24 satellites. However, the system currently employs more satellites than specified in the nominal constellation, and at the time of writing, the GPS constellation consists of 29 Block II/IIA/IIR satellites (US Naval Observatory, 2006).

All the satellites are inclined at $55^{\circ}$ to the equator and are orbiting the Earth every 11 hours and 58 minutes at a height of $20180 \mathrm{~km}$ on six different orbital planes [2]. These satellites are orientated in such a way that from any place on the Earth, at any time, at least four satellites are available for navigational purposes as shown in Fig. 1.1. As the demand for

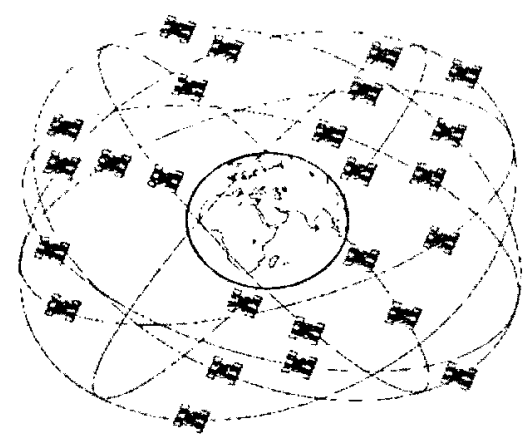

Figure 1.1: GPS Satellites Orbiting the Earth,taken from [6].

the use of GPS is increasing, the higher performance and accuracy are required. Although the degree of accuracy is highly application dependent (like life threatening applications), the fundamental aspects of positioning remain unchanged. GPS uses pseudoranges, derived from the broadcast satellite signals, to locate a user's position. The pseudorange is measured 
either by travel time of the coded signal and multiplying it by its velocity or by measuring the phase of the signal. In both cases the clocks of the receiver and the satellite are employed. Since these clocks are never perfectly synchronized, instead of true ranges, pseudoranges are obtained where the synchronization error (denoted as clock error) is taken into account [3]. GPS offers two kinds of services. the Precise Positioning Service (PPS) and the Standard Positioning Service (SPS). PPS includes a feature, called Anti-Spoofing (AS), and can be accessed only by DoD-authorized users equipped with appropriate encryption keys. However, SPS is open to all civil users and is used in this research.

\subsection{GPS Architecture}

The all weather global system consists of three segments:

- the space segment, consisting of satellites which broadcast signals

- the control segment, steering the whole system

- the user segment, including the many types of receivers.

\subsubsection{Space Segment}

The space segment of the system includes the constellation of GPS satellites. These space vehicles (SVs) transmit radio signals from space to the users. Each satellite has four atomic clocks on-board, but only one of them is being used to generate the fundamental L-band frequency of $10.23 \mathrm{MHz}$. From this findamental frequency two other carrier frequencies are derived. The L1 and L2 frequencies are generated by multiplying the fundamental frequency by 154 and 120 respectively [5].

- $\mathrm{L} 1=1575.42 \mathrm{MHz}, \lambda=19 \mathrm{~cm}$

- $\mathrm{L} 2=1227.60 \mathrm{MHz}, \quad \lambda=24.4 \mathrm{~cm}$

Fig. 1.2 shows detailed block diagram of the transmitted signal. The minimum signal 


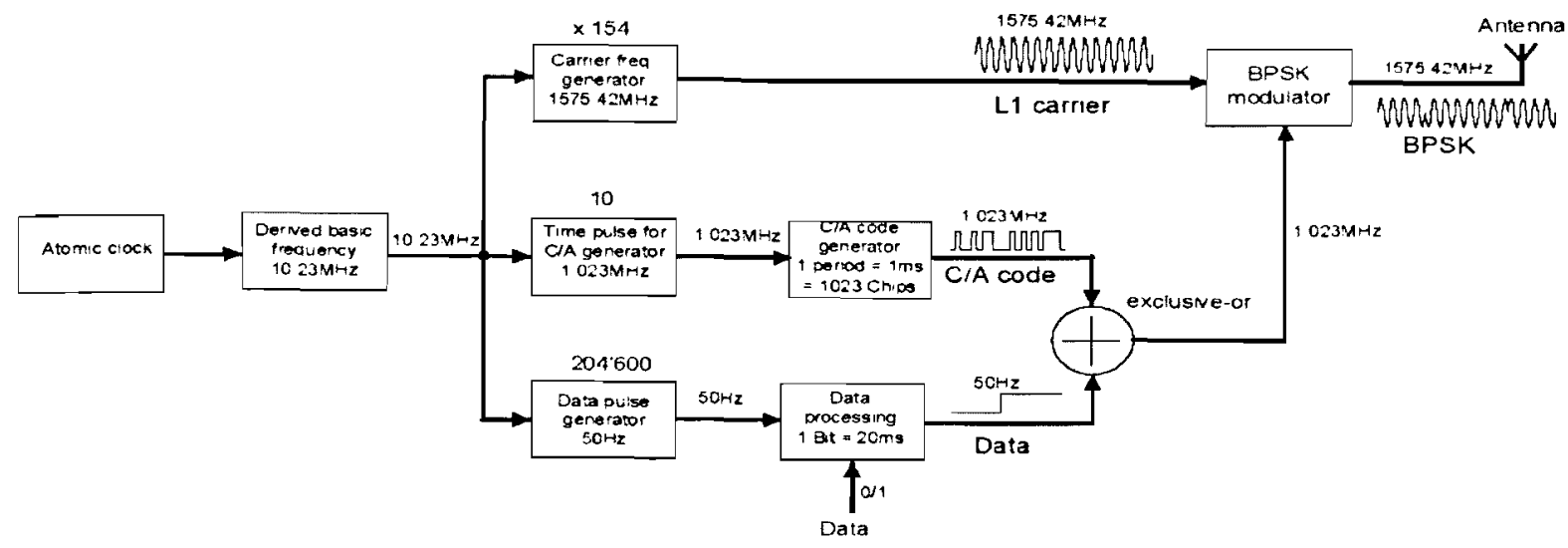

Figure 1.2: Detailed GPS Transmittcd Signal, C/A on L1 Band.

strength received on Earth is approximately $-158 \mathrm{dBW}$ to $-160 \mathrm{dBW}$ [6]. Every SV transmits its exact position and its precise on board clock time to the Earth at rate of L1 frequency.

\section{Navigation Message}

The navigation message is a continuous stream of data transmitted at $50 \mathrm{bps}$, as illustrated in Fig. 1.2. Each satellite relays the following information to Earth:

- system time and clock correction values

- its own highly accurate orbital data (ephemeris)

- approximate orbital data for all other satellites (almanac)

- system health (the whole satellite system is working properly or not)

The navigation message is needed to calculate the current position of the satellites and to determine signal transit times. The $\mathrm{C} / \mathrm{A}$ (coarse acquisition) code is a bi-phase coded signal which changes the carrier phase between 0 and $\pi$ at a rate of $1.023 \mathrm{MHz}$. The navigation data bit is also bi-phase code, but its rate is only $50 \mathrm{~Hz}$, or each data bit is $20 \mathrm{~ms}$ long. Since the $\mathrm{C} / \mathrm{A}$ code is $1 \mathrm{~ms}$, there are $20 \mathrm{C} / \mathrm{A}$ codes in one data bit. Thus, in one data bit all 20 $\mathrm{C} / \mathrm{A}$ codes have the same phase. 


\subsubsection{Control Segment}

The control system consists of a master control station, monitor stations, and ground control satiations. The main operational tasks of the control segment are $[3,6]$ :

- observing the movement of the satellites and computing orbital data (ephemeris)

- monitoring the satellite clocks and predicting their behavior

- synchronizing on board satellite time

- relaying precise orbital data received from satellites in communication

- relaying the approximate orbital data of all satellites (almanac)

- checking the satellite health and clock errors.

The control segment is also responsible for SA (selective availability) to degrade the system's positional accuracy for civil use.

\subsubsection{User Segment}

User segment includes user hardware and processing software for positioning, navigation, and timing applications. Today, GPS receivers are routinely being used to conduct all types of land and geodetic control surveys. Nowadays, most of the receivers can provide a good position approximation (less than $15 \mathrm{~m}$ horizontally) in an open sky, which most of the time is not the case. In an urban area sucki as downtown Toronto, the accuracy and precision of the computed positions will decrease mainly due to multipath error. The multipath could cause an error in the order of hundreds of meter horizontally.

GPS reccivers track and decode the signals from the satellites. A GPS receiver computes the location of the satellites based on their ephemeris and also measures the distance to the satellites based on the travel time of the radio signals. The recciver then deduces its own location based on a simple mathematical principle called trilateration in three-dimensional space. Accurate timing is the key to measuring distance to satellites. Atomic clocks carried 
on board the satellites are synchronized. In order to use inexpensive quartz oscillators, the receivers can utilize an extra satellite range measurement. With the distance measurements from at least four satellites, not only can the receiver calculate its position, but the receiver can also remove its clock bias (refer to chapter 2 for more details).

\subsection{Objective of the Thesis}

Multipath mitigation of the GPS signals both in terms of theory and application have been of the interest for many years. In this thesis we developed an algorithm which post-processes the signals and positions, from an inexpensive receiver operating only on L1 frequency, to decrease the standard deviation of the computed positions.

Wavelet analysis was used in two different stages to mitigate the effect of multipath and noise associated with the received data. Fig. 1.3 shows the block diagram of the proposed algorithm.

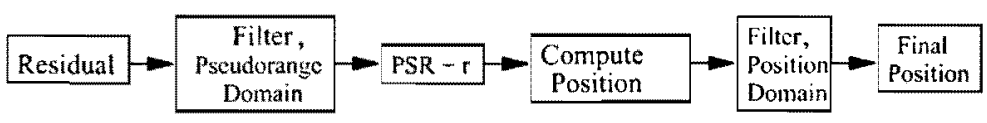

Figure 1.3: Block Diagram of the Proposed Algorithm.

\subsection{Main Contribution of the Thesis}

The blocks which are shaded in Fig. 1.3 are the author's main contributions, which are broken down to:

- study of GPS system and signal in details

- investigate wavelets for GPS multipath and signal processing; the discussion is necessary to build background for the proposed threshold value

- propose a novel threshold value based on eigenvalues of the wavelet coefficients

- develop adaptive filter level selection procedure 
- to identify and eliminate low velocities that exist among computed positions

- do performance evaluation using real data, and validation of the proposed algorithm

\subsection{Organization of the Thesis}

This thesis is organized in six chapters. In Chapter 1 we review at basics of the GPS. More specifically the GPS architecture is reviewed. GPS signal structure and characteristics are discussed in Chapter 2. In this chapter we study positioning, C/A code, and GPS errors. Chapter 3 is about wavelets. An introductory part on wavelets is presented first, and then we analyze noise reduction and filtering procedures. Multipath mitigation strategies are discussed in Chapter 4. In this chapter entropy and proposed algorithm are discussed in details. Chapter 5 is assigned to the results, and finally, Chapter 6 is the conclusion of the thesis. 


\section{Chapter 2}

\section{GPS Signal Structure and Characteristics}

The accuracy and precision of the computed positions in a receiver mainly depend on the location of the user. If the location is in an urban canyon area (such as downtown Toronto), the distance between the user and the satellites play an important rule in calculating the coordinates. The distance is calculated from the basic equation of velocity (i.e., speed of the light) times the traveling time. Therefore, the main source of error is hidden in the time. The transmitted signals from SVs will be affected by the channel characteristics which are mainly the atmospheric errors, including tropospheric and ionospheric delays, and multipath error. All these errors, plus the other sources of errors such as receiver clock drift, or thermal noises will show themselves as timing errors, which will consequently be converted to the ranging errors.

\subsection{Computing a Position in 3-D}

Measuring signal transit time and knowing the distance to a satellite are still not enough to calculate one's position in three dimensional plane. To achieve this, four independent transit time measurements are required. That is, four different satellites are needed to locate a receiver (user). 


\subsubsection{Determining a Position on a Plane}

With only one satellite in view, the user has the pseudorange value of $P$. Then, the position of the user will be on the circumference of a circle having radius $\mathrm{P}$. This radius is at least $20000 \mathrm{~km}$ long; therefore, imagine where could the user be on the circle. To narrow down the search, the user could use two pseudoranges $\left(\mathrm{P}_{1}, \mathrm{P}_{2}\right)$ from two SVs. In this case, the position of the user is where the two circles of the radius $P_{1}$ and $P_{2}$ intersect. Obviously, there are two intersection points in that case, but the point above the satellites will be ignored as shown in Fig. 2.1.

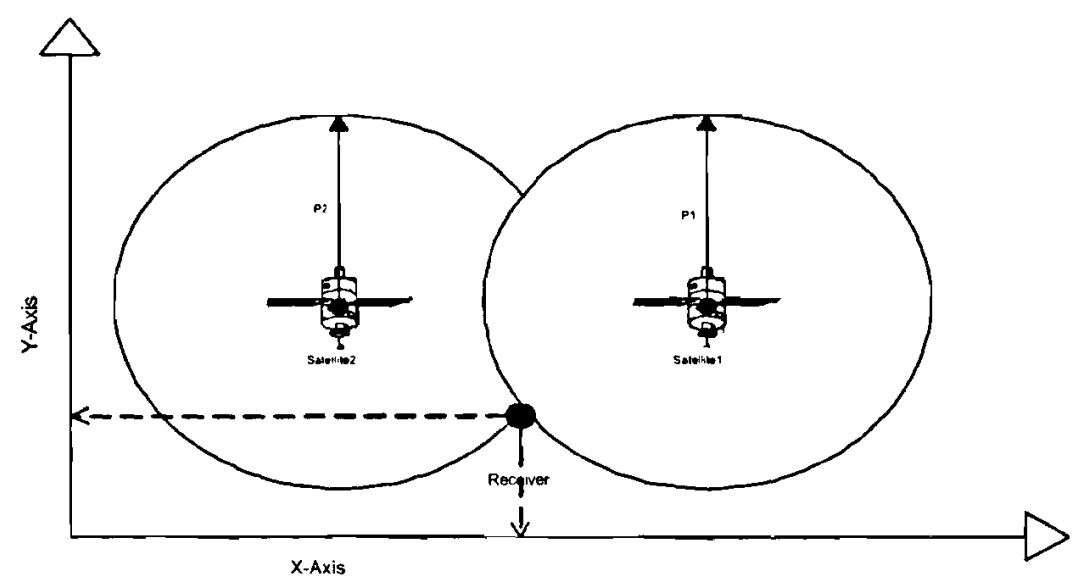

Figure 2.1: Positioning on 2 Dimcnsional Planc.

\subsubsection{Determining a Position on Three Dimensional Space}

While two satellites are sufficient to locate a receiver in two dimensional plane (with latitude and longitude), a position has to be determined in three dimensional space (with latitude,longitude, and height). Therefore, a third satellite is needed to fully position a user. With three pseudoranges, the position of the user will be on the surface of a sphere, which results from the intersection of the three spheres as illustrated in Fig. 2.2. It was stated [3] that three pseudoranges are needed to compute a position. This is true provided that there is no timing errors. In other words, the atomic clock on-board and the receiver clock need to be synchronized. However, this is not the case. There is no synchronization between 


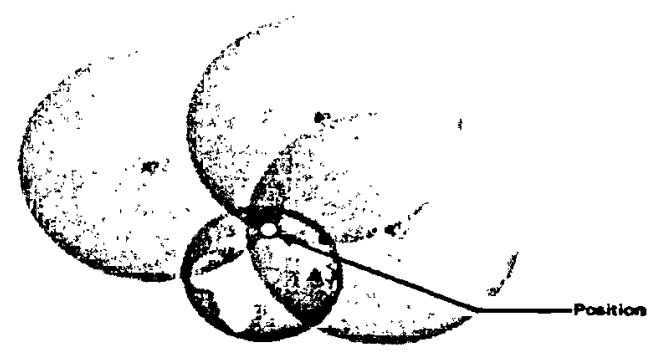

Figure 2.2: Positioning on 3 Dimensional Space, taken from [6].

a receiver and satellite clocks. Even if the error difference between the receiver clock and the transmit time is $1 \mu \mathrm{s}$, it produces a position error of $300 \mathrm{~m}$. To overcome this problem the timing error is considered to be an unknown; consequently, there are four unknowns (longitude, latitude, hight, and time error) to be solved in a 3-D positioning.

\subsubsection{Position Calculations}

In order for a GPS receiver to determine its position, it has to receive time signals from four different satellites to calculate signal transit times $\Delta t_{1}$ to $\Delta t_{4}$. The range of the user from the four satellites $R_{1}, R_{2}, R_{3}$, and $R_{4}$ can be determined with the help of signal transit times, $\Delta t_{1}$ to $\Delta t_{4}$, between the four satellites and the receiver. Since the locations of the four satellites are known, as mentioned in section 1.2.1, the user co-ordinates can be calculated [6]. Due to the atomic clocks on-board, the time at which the satellite signal is transmitted is known precisely. All satellite clocks are synchronized based on universal time co-ordinates (UTC) [2]. In contrast, the receiver clock is not synchronized to UTC and is therefore, slower or faster by $\Delta t_{0}$. The sign of $\Delta t_{0}$ is positive when the user clock is faster and negative otherwise. The resultant time error $\Delta t_{0}$ causes inaccuracies in the measurement of signal transit time and the distance $R$ (true range of the satellite from user) [6],

$$
\begin{array}{r}
\Delta t_{\text {measured }}=\Delta t+\Delta t_{0} \Rightarrow P S R=\Delta t_{\text {measured }} \cdot c \\
P S R=\left(\Delta t+\Delta t_{0}\right) \cdot c \\
P S R=R+\Delta t_{0} \cdot c \Rightarrow P S R=R+r,
\end{array}
$$


where $\Delta t$ is signal transit time from satellite to receiver, $\Delta t_{0}$ is the resultant time error, which is the difference between satellite and receiver clock plus the time delay due to errors (such as multipath), $c$ is the speed of the light, and $P S R$ represents the pseudorange. In Eq. (2.3) the error distance $r$ is the distance due to the resultant time error $\Delta t_{0}$. One of the main contributions in this thesis is to derive approximate value for $r$.

Since the $X, Y, Z$ coordinates of the SVs are known to users, the true distance $R$ can be calculated as:

$$
R_{i}=\sqrt{\left(X_{s_{2}}-X_{u_{i}}\right)^{2}+\left(Y_{s_{2}}-Y_{u_{i}}\right)^{2}+\left(Z_{s_{i}}-Z_{u_{i}}\right)^{2}}
$$

where $X_{s_{i}}$ is the $X$ coordinate of the known satellite and $X_{u_{i}}$ is the user coordinate (unknown). The subscripts si and ui in $X_{s_{2}}$ and $X_{u_{\imath}}$ are the index values. Eq. (2.4) is a general equation representing a distance. It could be used in a stationary or a dynamic mode. Since the number of satellites must be at least four to fully locate a user position, the indexing $i$ is from 1 to 4. By replacing Eq. (2.4) in Eq. (2.3), the resultant pseudoranges will have the form of Eq. (2.5).

$$
P R S_{i}=\sqrt{\left(X_{s_{i}}-X_{u_{i}}\right)^{2}+\left(Y_{s_{i}}-Y_{u_{1}}\right)^{2}+\left(Z_{s_{i}}-Z_{u_{i}}\right)^{2}}+c . \Delta t_{0}
$$

Eq. (2.5) represents a set of non-linear equations with four unknowns. One method to solve the system of non-linear equations is to linearize them first. The Taylor series is a known method to be used for this problem. The general form of the Taylor series is as follows:

$$
f(x)=f\left(x_{0}\right)+\frac{f^{\prime}\left(x_{0}\right)}{1 !} \cdot \Delta x+\frac{f^{\prime \prime}\left(x_{0}\right)^{2}}{2 !} \cdot \Delta x+\frac{f^{\prime \prime \prime}\left(x_{0}\right)^{3}}{3 !} \cdot \Delta x+\ldots,
$$

where $\Delta x$ is the error or deviation from the true value of $x\left(\Delta x=x-x_{0}\right)$ and $x_{0}$ is an initial or guessing value. The same principle is used to approximate a solution for the above mentioned non-linear system. However, as the value of error decreases at the high order of Taylor series in navigation applications, it is sufficient to accept the Taylor series up to its first order for our purpose [2].

$$
f(x)=f\left(x_{0}\right)+\frac{f^{\prime}\left(x_{0}\right)}{1 !} \cdot \Delta x
$$


Eq. (2.7) is the simplified version of the Taylor series to be used to solve the non-linear system of equations expressed in Eq. (2.5).

Therefore, the unknown terms $X_{u_{\imath}}, Y_{u_{\imath}}$, and $Z_{u_{\imath}}$ in the Eq. (2.5) can be replaced with the initial values of $[2,5]$ :

$$
\begin{gathered}
X_{u_{\imath}}=X_{g}+\Delta x \\
Y_{u_{\imath}}=Y_{g}+\Delta y \\
Z_{u_{i}}=Z_{g}+\Delta z
\end{gathered}
$$

where the $X_{g}$ is an initial or guessing value for the $X$ coordinate of the user. Consequently, Eq. (2.4) will be transformed to Eq. (2.9).

$$
R_{g_{2}}=\sqrt{\left(X_{s_{\imath}}-X_{g_{\imath}}\right)^{2}+\left(Y_{s_{i}}-Y_{g_{\imath}}\right)^{2}+\left(Z_{s_{i}}-Z_{g_{i}}\right)^{2}}
$$

where $R_{g_{i}}$ is the guessing distance between a user and a satellite. By applying the Taylor series on Eq. (2.9), the pseudoranges expressed in Eq. (2.5) will be transformed to Eq. $(2.10)$

$$
P R S_{i}=R_{g_{i}}+\frac{\partial\left(R_{g_{2}}\right)}{\partial x} \cdot \Delta x+\frac{\partial\left(R_{g_{\imath}}\right)}{\partial y} \cdot \Delta y+\frac{\partial\left(R_{g_{2}}\right)}{\partial z} \cdot \Delta z+c \cdot \Delta t_{0}
$$

where,

$$
\frac{\partial\left(R_{g_{2}}\right)}{\partial x}=\frac{X_{g}-X_{s_{2}}}{R_{g_{i}}}
$$

Therefore, Eq. (2.12) is a final representation of the pseudoranges.

$$
P R S_{i}=R_{g_{i}}+\frac{\left(X_{g}-X_{s_{2}}\right)}{R_{g_{2}}} \cdot \Delta x+\frac{\left(Y_{g}-Y_{s_{2}}\right)}{R_{g_{i}}} \cdot \Delta y+\frac{\left(Z_{g}-Z_{s_{2}}\right)}{R_{g_{i}}} \cdot \Delta z+c . \Delta t_{0}
$$

Now, we can solve the linear system of equations with the unknowns $\Delta x, \Delta y, \Delta z$, and $\Delta t_{0}$ $[2,5]$.

$$
\left[\begin{array}{l}
P S R_{1}-R_{g_{1}} \\
P S R_{2}-R_{g_{2}} \\
P S R_{3}-R_{g_{3}} \\
P S R_{4}-R_{g_{4}}
\end{array}\right]=\left[\begin{array}{cccc}
\frac{X_{g}-X_{s_{1}}}{R_{g}} & \frac{Y_{g}-Y_{s_{1}}}{R_{g_{1}}} & \frac{Z_{g}-Z_{s_{1}}}{R_{g}} & c \\
\frac{X_{g}-X_{s_{2}}}{R_{s_{2}}} & \frac{Y_{g}-Y_{s_{2}}}{R_{g_{2}}} & \frac{Z_{g}-Z_{s_{2}}}{R_{s_{2}}} & c \\
\frac{X_{g}-X_{s_{1}}}{R_{g_{3}}} & \frac{Y_{g}-Y_{s_{3}}}{R_{g_{3}}} & \frac{Z_{g}-Z_{s_{3}}}{R_{g_{3}}} & c \\
\frac{X_{g}-X_{s_{1}}}{R_{g_{4}}} & \frac{Y_{g}-Y_{s_{1}}}{R_{g_{4}}} & \frac{Z_{g}-Z_{s_{1}}}{R_{g_{4}}} & c
\end{array}\right] \cdot\left[\begin{array}{c}
\Delta x \\
\Delta y \\
\Delta z \\
\Delta t_{0}
\end{array}\right]
$$




$$
\Rightarrow\left[\begin{array}{c}
\Delta x \\
\Delta y \\
\Delta z \\
\Delta t_{0}
\end{array}\right]=\left[\begin{array}{llll}
\frac{X_{g}-X_{s_{1}}}{R_{g}} & \frac{Y_{g}-Y_{s_{1}}}{R_{g_{1}}} & \frac{Z_{g}-Z_{s_{1}}}{R_{g}} & c \\
\frac{X_{g}-X_{s_{2}}}{R_{s_{2}}} & \frac{Y_{g}-Y_{s_{2}}}{R_{g_{2}}} & \frac{Z_{g}-Z_{s_{2}}}{R_{g}} & c \\
\frac{X_{g}-X_{s_{1}}}{R_{s_{3}}} & \frac{Y_{g}-Y_{s_{3}}}{R_{s_{3}}} & \frac{Z_{g}-Z_{s_{3}}}{R_{s_{3}}} & c \\
\frac{X_{g}-X_{s_{1}}}{R_{g_{4}}} & \frac{Y_{g}-Y_{s_{4}}}{R_{g_{4}}} & \frac{Z_{g}-Z_{s_{1}}}{R_{g_{4}}} & c
\end{array}\right]^{-1} \cdot\left[\begin{array}{l}
P S R_{1}-R_{g_{1}} \\
P S R_{2}-R_{g_{2}} \\
P S R_{3}-R_{g_{3}} \\
P S R_{4}-R_{g_{4}}
\end{array}\right]
$$

The solutions of the unknowns are used to update the initial positions $X_{g}, Y_{g}$, and $Z_{g}$ in accordance with Eq. (2.15).

$$
\begin{gathered}
X_{\text {new }_{i}}=X_{\text {old }_{g}}+\Delta x \\
Y_{\text {new }_{i}}=Y_{\text {old }_{g}}+\Delta y \\
Z_{\text {new }_{i}}=Z_{\text {old }_{g}}+\Delta z
\end{gathered}
$$

In case there is no solution to the system of the equations, we could use another satellite data. For example, in case we have six satellites in view, we could replace $P S R_{5}$ and $P S R_{6}$ with the $P S R_{1}$ and $P S R_{2}$. The estimated values $X_{n e w_{i}}, Y_{n e w_{i}}$, and $Z_{n e w_{i}}$ can now be entered into the set of equations given by Eq. (2.14) using normal iterative process until error components $\Delta x, \Delta y$, and $\Delta z$ are smaller than the desired error value (i.e., $10 \mathrm{~cm}$ ) [6]. Usually, three to five iterations would be sufficient to get an error less than $10 \mathrm{~cm}[3]$.

\subsection{GPS Signal Structure}

Because GPS signal falls into the category of signals known as spread spectrum signaling, the fundamentals of spread spectrum signaling are introduced next.. The chapter continues with a detailed description of the GPS signal structure for civil coarse acquisition (C/A code) signal.

\subsubsection{Fundamentals of Spread Spectrum Signaling}

Spread spectrum signaling in its most fundamental form is a method of taking a data signal $D(t)$ of bandwidth $B_{d}$ that is modulated on a sinusoidal carrier to form $d(t)$, and then spreading its bandwidth to a much larger value $B_{s}$, where $B_{s} \gg B_{d}$. The bandwidth spreding can be accomplished by multiplying the data modulated carrier by a wide bandwidth 
spreading waveform $s(t)[2]$.

The binary data bit stream $D(t)$ with values $D= \pm 1$ and clock rate $f_{d}$ is first modulated on a carrier of power $P_{d}$ to form the narrow bandwidth signal:

$$
d(t)=D(t) \sqrt{2 P_{d}} \cos \left(\omega_{d} t\right)
$$

This narrow bandwidth signal of bandwidth $B_{d}$ is then spread in bandwidth by a binary pseudorandom signal $s(t)$, where $s(t)= \pm 1$ and has a clock rate $f_{c}$ that greatly exceeds the data bit rate, i.e. $f_{c}>>f_{d}[2]$.

The spread spectrum signal then has the following form:

$$
s_{o}(t)=s(t) d(t)=s(t) D(t) \sqrt{2 P_{d}} \cos \left(\omega_{d} t\right)
$$

where the spreading signal is as follows:

$$
s(t)=\sum_{n=-\infty}^{\infty} S_{n} p\left(t-n T_{c}\right)
$$

and $p(t)$ is a rectangular unit pulse over the interval $\left(0, T_{c}=1 / f_{c}\right)$, and $S_{n}$ is a random or pseudorandom sequence $S_{n}= \pm 1[2]$.

\subsection{GPS Signal (C/A Code) Characteristics}

As mentioned previously, each GPS satellite transmits signals on two L-band frequencies of $f_{L 1}$ at $1575.42 \mathrm{MHz}$ and $f_{L 2}$ at $1227.60 \mathrm{MHz}$. The signal can be split into three parts. The first part is the carriers which are the carrier waves with $f_{L 1}$ or $f_{L 2}$ frequency. The second part of the signal is the navigation data. The navigation data provides the means for the receiver to determine the location of the satellite at the time of signal transmission. The navigation message, which contains information about the satellites, GPS time, clock behavior, and system status, is modulated on both the L1 and L2 carriers at a chip rate of 50 bits per second (bps) with a bit duration of $20 \mathrm{~ms}$. These information are transmitted to all satellites from the ground stations in the GPS Control Segment. The third part, spreading sequence, was briefly explained in section 2.2.1. 
Each satellite has two unique spreading sequences or codes. The first one is the coarse acquisition (C/A) pseudorandom noise (PRN) code sequence modulating the L1 carrier phase, and the other one is the encrypted precision code $(\mathrm{P}(\mathrm{Y}))$ sequence modulating both the L1 and L2 carrier phases. L1 is Binary-Phase Shift Key (BPSK) modulated with a pseudo random noise $1.023 \mathrm{MHz}$ code known as the Coarse/Acquisition (C/A) code. This C/A code sequence repeats every millisecond. The transmitted PRN code sequence is actually the modulo- 2 addition of the $50 \mathrm{~Hz}$ navigation message and the $\mathrm{C} / \mathrm{A}$ code [5].

\subsubsection{C/A Code Generation}

The signal transmitted from satellite $k$ can be described as $[2,4]$ :

$$
\begin{aligned}
s^{k}(t)=\sqrt{2 P_{c}}\left(C^{k}(t) \oplus D^{k}(t)\right) \cos \left(2 \pi f_{L 1} t\right) & +\sqrt{2 P_{P L 1}}\left(P^{k}(t) \oplus D^{k}(t)\right) \sin \left(2 \pi f_{L 1} t\right) \\
& +\sqrt{2 P_{P L 2}}\left(P^{k}(t) \oplus D^{k}(t)\right) \sin \left(2 \pi f_{L 2} t\right)
\end{aligned}
$$

where $P_{C}, P_{P L 1}$, and $P_{P L 2}$ are the powers of signals with C/A code, $C^{k}$ is the C/A code sequence assigned to satellite number $k, P^{k}$ is the $\mathrm{P}(\mathrm{Y})$ code sequence assigned to satellite number $k, D^{k}$ is the navigation data sequence, and $f_{L 1}$ and $f_{L 2}$ are the carrier frequencies of L1 and L2 respectively.

The spreading sequences used as C/A codes in GPS belong to a unique set of sequences. They are often referred to as Gold codes. They are also referred to as pseudo-random noise sequences or simply PRN sequences because of their characteristics. The generation of the Gold codes is sketched in Fig. 2.3. As illustrated in there, the C/A code generator contains two shift registers known as G1 and G2. Each of these shift registers has ten states generating sequences of length 1023 chip. The two resulting 1023 chip long sequences are modulo-2 added to generate a 1023 chip long C/A code. For every $1023^{\text {rd }}$ chip, the shift registers are reset with all ones, making the code to start over. G1 always has a feed back configuration with the following polynomial [2]:

$$
1+X^{3}+X^{10},
$$




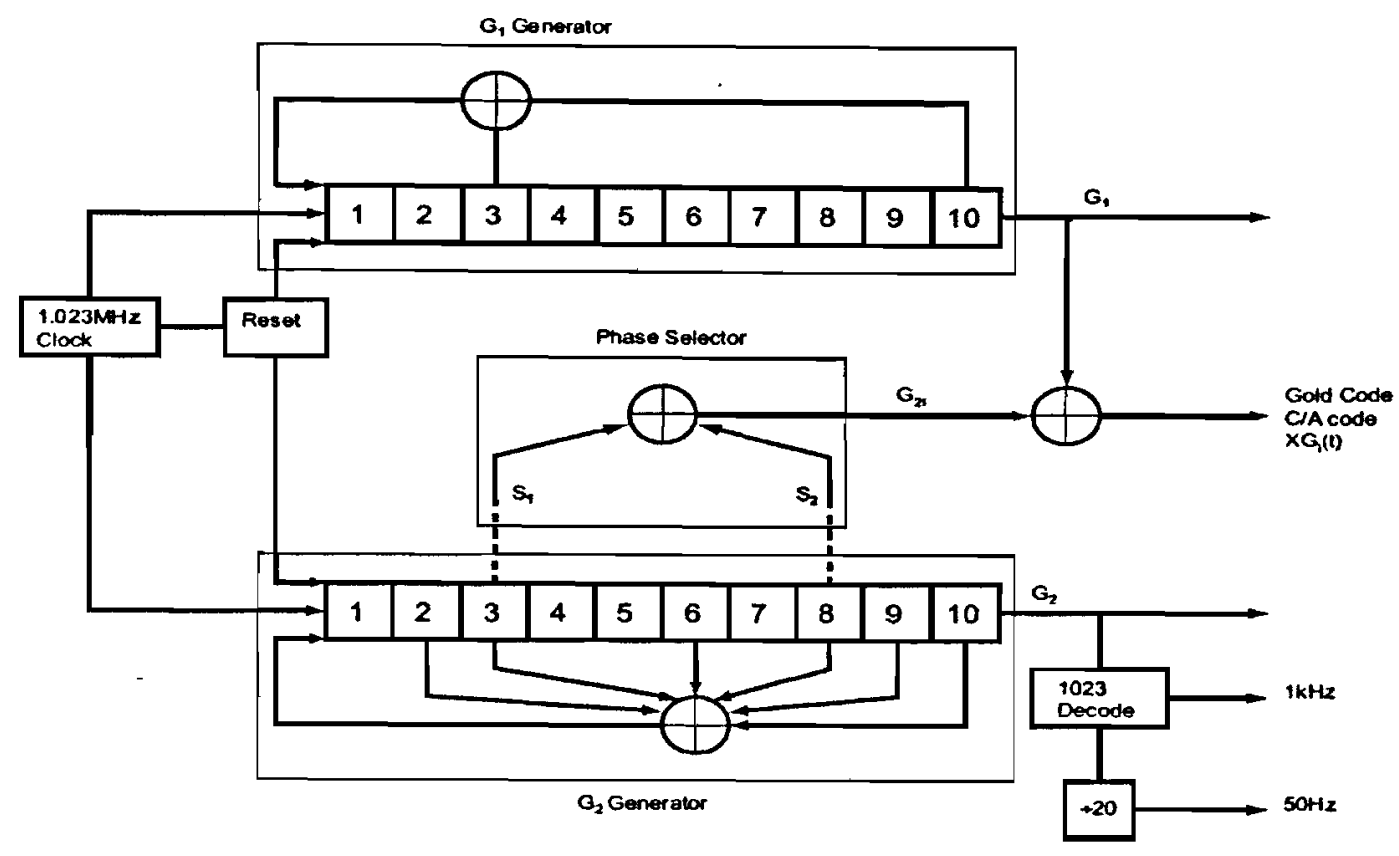

Figure 2.3: C/A Code Generation, taken from [8].

which states that the registers three and ten are inputs to the XOR gate and the output of the gate goes back into the register one. In the same way, the G2 register has the following polynomial [2]:

$$
1+X^{2}+X^{3}+X^{6}+X^{8}+X^{9}+X^{10} .
$$

The G1 always supplies its output, but the G2 supplies two of its states to a modulo-2 adder to generate its output. The selection of states for the modulo-2 adder is called the phase selection. Fig. A.1 (given in Appendix A) shows the combination of the phase selections for each $\mathrm{C} / \mathrm{A}$ code. It also shows the first 10 chips of each code in octal representation. Fig. 2.4 shows a sample generated (with Matlab) C/A code (PRN 6).

\subsubsection{Auto and Cross Correlation of the C/A Codes}

The most important characteristics of the $\mathrm{C} / \mathrm{A}$ codes are their correlation properties. Based on the design of the Gold codes, all the C/A codes are nearly uncorrelated with themselves, 


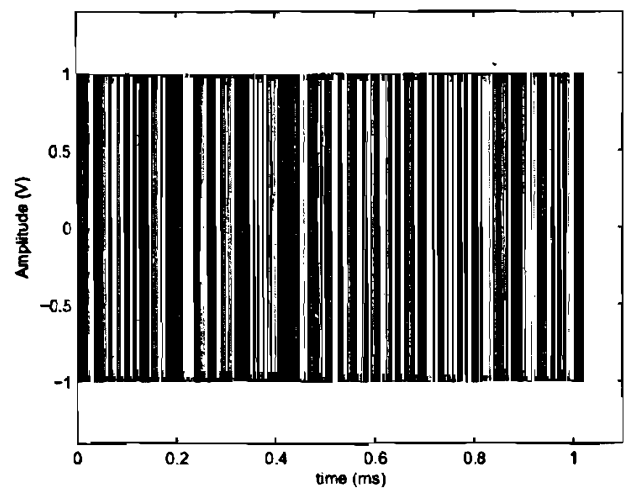

(a) Generated C/A Code (PRN 6).

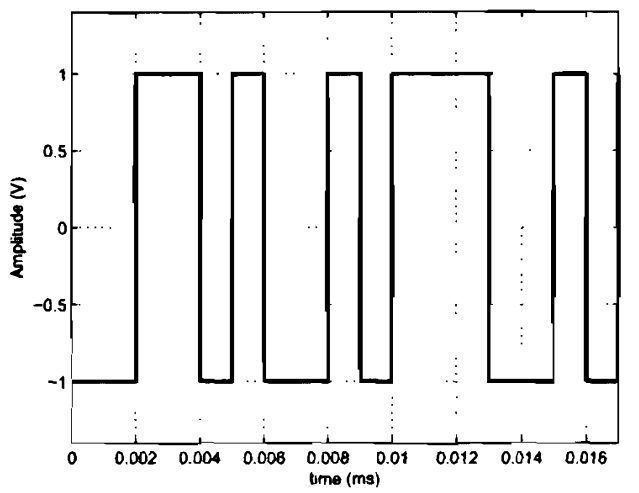

(b) Partial (Zoomed) Part of the C/A Code.

Figure 2.4: The First $1023 \mathrm{C} / \mathrm{A}$ (PRN6) Code.

except at zero lag, and nearly uncorrelated with each other. Fig. 2.5(a) shows high correla-
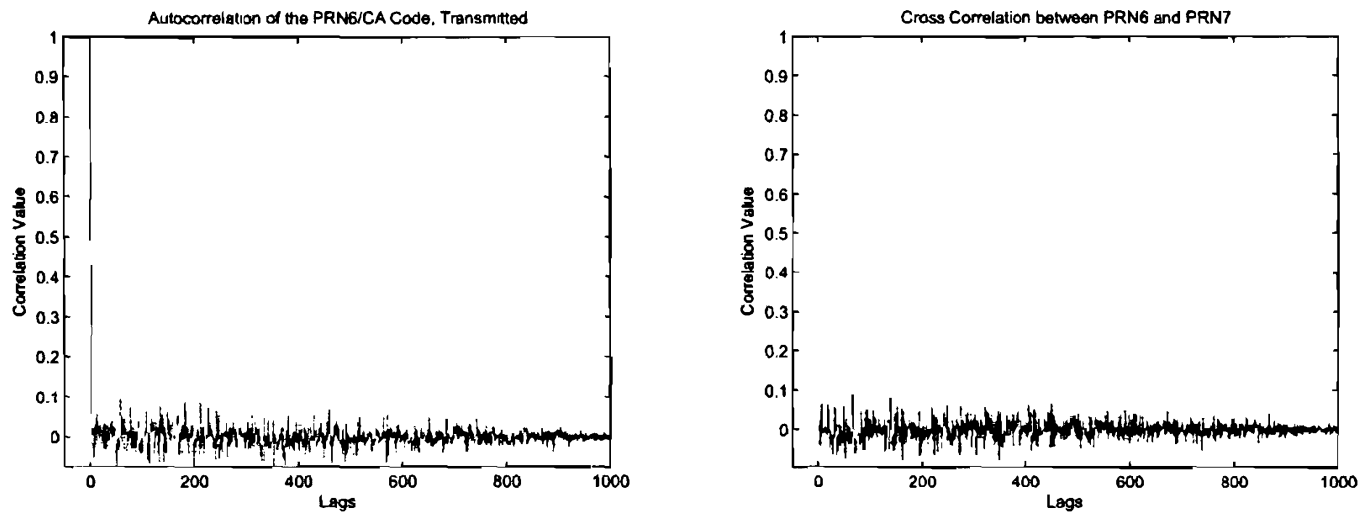

(a) Auto-correlation of the C/A Code (PRN 6). (b) Cross-correlation between PRN6 and PRN7.

Figure 2.5: Auto and Cross correlation of the C/A Code (PRN6 and PRN7).

tion at zero lag when correlating with the same C/A code, while Fig. 2.5(b) illustrates low correlation when correlating with another C/A code (PRN6 and PRN7).

Correlation property of the $\mathrm{C} / \mathrm{A}$ codes is used in receivers to find out which satellite is being tracked. The principle is that receivers generate their own PRNs and try to match them with the received signals. Therefore, the high value of correlation at zero lag makes it easy to find out when two similar codes are perfectly aligned. 


\subsubsection{The Transmitted Satellite Signals}

As shown in Fig. 2.6, the transmitted satellite signal (on L1, C/A code) consists of L1carrier, navigation data, and the $\mathrm{C} / \mathrm{A}$ ranging code. The description of the navigation data is mentioned in sections 1.2.1 and 2.3. The transmitted signal undergoes changes on the way

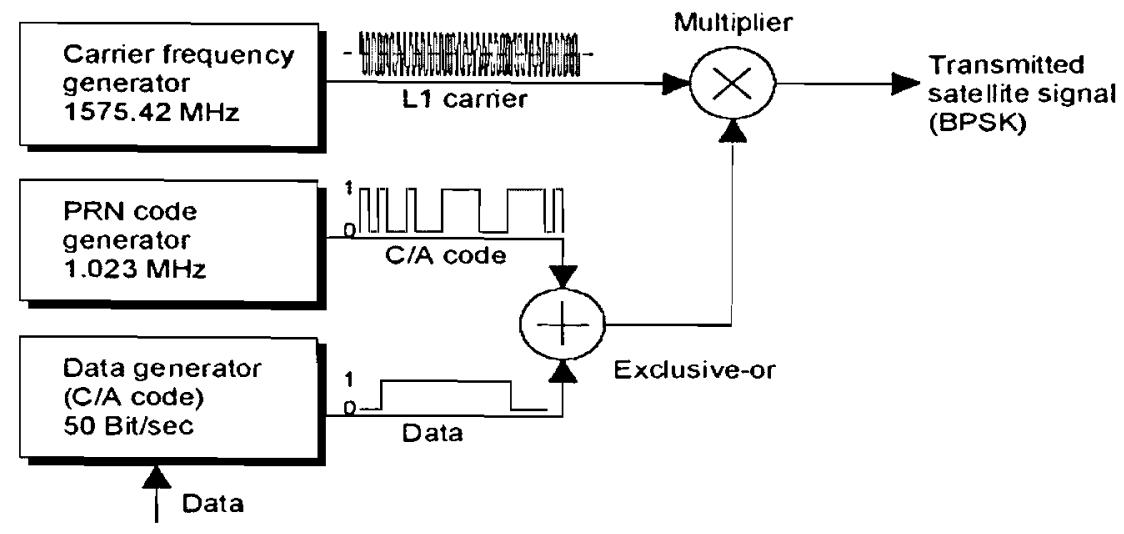

Figure 2.6: GPS Transmittcd Signal, C/A on L1 Band.

to a receiver on the Earth. These changes take place in the space (mainly passing through the troposphere and ionosphere layers) and on the Earth (equipment and multipath errors). Therefore, the GPS signal incurs losses during its propagation from the satellite to the Earth.

\section{Signal Power}

The line-of-sight GPS signals received by users are $-160 \mathrm{dBW}$ in strength [2]. The dimension $\mathrm{dBW}$ denotes the ratio of power relative to 1 Watt. A value of $-160 \mathrm{dBW}$ corresponds to -130 $\mathrm{dBm}$, which denotes power with respect to $1 \mathrm{~mW}$. The GPS signals are not to exceed the internationally agreed power values set to avoid interference with other users and systems [10]. The noise power of the broadcast GPS signal is:

$$
N_{p}=k T B
$$

where $N_{p}$ is the noise power in Watt, $k$ is the Boltzmans constant $\left(1.38066 \times 10^{-23} \mathrm{~J} / \mathrm{K}\right), T$ is the equivalent noise temperature (nominally $273 \mathrm{~K}$ ), and $B$ is the bandwidth in units of $\mathrm{Hz}$. Within L1 C/A main lobes of a $2.046 \mathrm{MHz}$ bandwidth, which contains about $90 \%$ of 
the signal power, the noise power is approximately $-14.1 \mathrm{dBW}$. In poor signal conditions the power of the satellite signal is even further decreased. Metals and concrete near the receiver result in up to a $20 \mathrm{~dB}$ loss or more; plywood sheets may lead to a $3 \mathrm{~dB}$ loss; drywall to a loss of about $1 \mathrm{~dB}$; trees typically bring about a loss of 5 to $8 \mathrm{~dB}$ and up to $20 \mathrm{~dB}$ or even more, depending on the tree size and density of foliage [10]. Thus, the amount of signal attenuation depends on the structure of the material, its density, and how much material the signal passes through.

\subsection{Error Sources}

Error sources affecting the GPS signals can be categorized in four groups as follows:

1. Satellite originated errors: including hardware delay, speed of the light, ephemeris, and clock errors.

- Although each satellite has four atomic clocks on-board, a time error of just 10 ns creates an error of $3 \mathrm{~m}$.

- The position of a satellite is generally known only to within approximately 1 to $5 \mathrm{~m}$.

- The signals from satellite to the user travel at the speed of light. This slows down when traveling through the Ionosphere and Troposphere layers of the atmosphere, therefore, speed of the light can not considered to be constant.

2. Ionospheric and Tropospheric delays

- A major source of bias error is the delay of the GPS carrier signals as they pass through the layer of charged ions and free electrons known as the ionosphere. Varying in density and thickness as it rises and falls ( 50 to $500 \mathrm{~km}$ ) due to solar pressure and geomagnetic effects, the ionosphere layer can delay the GPS signals (code) by as much as $300 \mathrm{~ns}(100 \mathrm{~m})$ [2]. 
- GPS signal delays through the troposphere, the layer of atmosphere usually associated with changes in weather (from ground level up to 8 to $13 \mathrm{~km}$ ), are subject to local conditions and are difficult to model.

3. Geometric effect of the satellites

- Satellite geometry is the constellation of the satellites in the sky. If the four SV's needed to compute a position are close to each other, the accuracy and precision deteriorates and visa versa.

4. Receiver errors: including hardware delay, system noise, clock, and multipath errors.

- Among the receiver errors, multipath is the most difficult one to mitigate, since it is very hard to model it. Multipath interference, caused by local reflections of the GPS signal that mix with the desired signal, introduces varying bias errors in the order of nanoseconds.

\subsubsection{Received Signals}

The quality of the received signals, by a GPS receiver, highly depend on the environment where the receiver's antenna is placed, since GPS signals can arrive at the receiver via multiple paths. Although signal degradation caused by troposphere and ionosphere delays have the same degree of importance as the multipath error, the emphasis is on multipath mitigation in this thesis.

The transit time of a signal from a satellite to a receiver can be measured as the amount of shift required to align the $\mathrm{C} / \mathrm{A}$ code replica generated at the receiver with the signal received from the satellite. The received signal is identified and aligned with the receiver clock generated signal through the exploitation of the autocorrelation properties of the PRN codes. Multiplying the transit time by the speed of light results in the measured satellite-touser range. Timing errors between the receiver clock and the satellite clock from system time cause the observed range to differ from the geometric distance corresponding to the instants of transmission and reception of the satellite signal [10]. Therefore, the measured range is 
called the pseudorange. In addition to the clocks causing the pseudorange to differ from the geometric range, the pseudorange measurement contains various other error components as mentioned above. The effect of some of these errors or biases are taken into account and corrected by the receiver, like the effect of the receiver clock drift, which is included as an unknown in the calculation of the position, or the effect of the ionosphere, which is mitigated using ionospheric models. However, the effect of multipath, which is to delay the line-of-sight signal by some amount, is clifficult to mitigate. Multipath disturbane is largely influcneed by the receiver's antenna environment since satellite signals can arrive at a GPS receiver via multiple paths due to reflections from nearby trees, terrain, buildings, and vehicles. For the GPS C/A signal, this error can range from a few meters up to more than $100 \mathrm{~m}$, depending on the scenario [2].

\subsubsection{Multipath Error Modeling}

A multipath transmission takes place when a transmitted signal arrives at the receiver by two or more paths of different delays. In radio links, a signal can be received by direct path between the transmitting and the receiving antenna and also by reflections from other objects such as hills or buildings, and so on [11]. In each of these cases, the transmission channel can be represented as several channels in parallel, each with different relative attenuation and a different time delay. A simplified version of a direct GPS signal, Eq. (2.19), can be expressed as:

$$
S_{d}(t)=A P(t) \sin \left(w_{c} t\right)
$$

where $\Lambda$ is amplitude, $P(t)$ is the pseudo-code, and $w_{c}$ is the carrier frequency of the transmitted signal. The reflected signals could be modeled as:

$$
S_{m}(t)=\sum_{k=1}^{m} a_{k} \Lambda P\left(t-\delta_{k}\right) \sin \left(w_{c} t+\theta_{k}\right), \quad k=1, \ldots, m
$$

where $m$ represents the number of reflected signals, $a_{k}$ is the attenuation factor, $\delta_{k}$ is the delayed time, and $\theta_{k}$ is the phase shift caused by the reflection of the signal from any physical 
object. Therefore, the received GPS signal, $S(t)$ in Eq. (2.25), is a superposition of the direct plus the reflected signals.

$$
\begin{gathered}
S(t)=S_{d}(t)+S_{m}(t) \\
S(t)=A P(t) \sin \left(w_{c} t\right)+\sum_{k=1}^{m} a_{k} A P\left(t-\delta_{k}\right) \sin \left(w_{c} t+\theta_{k}\right)
\end{gathered}
$$

At the receiver, these multipath waves with randomly distributed amplitudes and phases combine to give a resultant signal. Therefore, a receiver at one location may have a signal that is much different from the signal at another location only a short distance away because of the change in the phase relationship among the incoming radio waves. This situation causes significant fluctuations in the signal amplitude. This phenomenon of random fluctuations in the received signal level is termed as fading [11]. If it is assumed that there is no direct path or line of sight (LOS) component, which is the case in most of the urban canyon areas, the received signal, $S(t)$, can be expressed as:

$$
S(t)=\sum_{k}^{m} a_{k} \cos \left(w_{c} t+\theta_{k}\right) .
$$

When there is relative motion between the transmitter and the receiver, Eq. (2.26) must be modified to include the effects of motion induced frequency and phase shifts. The $k^{\text {th }}$ reflected wave with amplitude $a_{k}$ and phase $\theta_{k}$ arrive at the receiver from an angle $\zeta_{k}$ relative to the direction of motion of antenna or the transmitter. The Doppler shift of this wave is given by:

$$
w_{d_{k}}=\frac{w_{c} v}{c} \cos \left(\zeta_{k}\right),
$$

where $v$ is the velocity of the mobile, $c$ is the speed of the light, and $\zeta_{k}$ is uniformly distributed over $[0,2 \pi]$. Therefore, the received signal now can be shown as:

$$
S(t)=\sum_{k=1}^{m} a_{k} \cos \left(w_{c} t+w_{d_{k}} t+\theta_{k}\right) .
$$

Obviously, in case of stationary process the value of $w_{d_{k}}$ will be zero. Figs. 2.7(a) and 2.7(b) show the generated received signals when the number of paths is simulated to be one and 


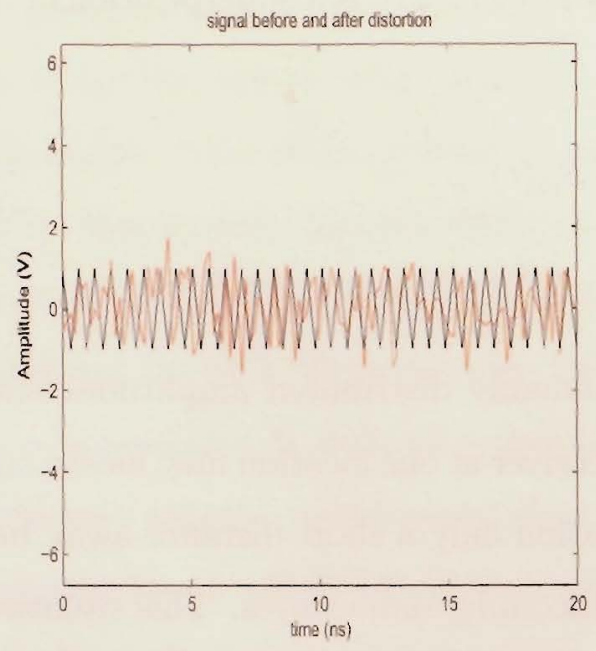

(a) One Added Path.

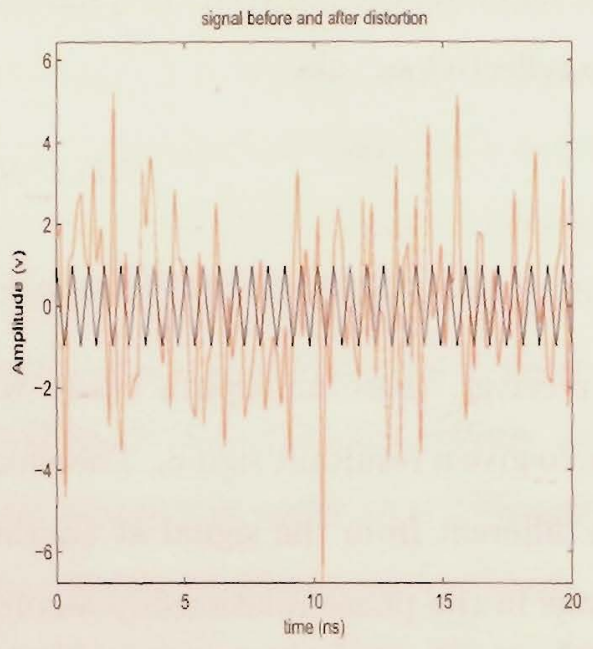

(b) Ten Added Paths.

Figure 2.7: Generated Multipath Signals.

ten respectively. In Fig. 2.7, the darker color wave (constant amplitude) is the direct and undistorted transmitted signal, while the more oscillatory wave is the corrupted signal with random amplitudes and phases (resultant of the multipaths signal).

In order to observe the effect of the multipath more closely, two C/A codes (PRN6 and PRN9) were generated. These two signals were then sent through a Rayleigh channel to observe the effect of multipaths. In this regard, Rayleigh fading is first discussed.

\section{Rayleigh Distribution}

The Rayleigh distribution usually arises when a two dimensional vector which has its two orthogonal components normally and independently distributed. The distribution may also arise in the case of random complex numbers whose real and imaginary components are normally and independently distributed. The absolute value of these numbers will then be Rayleigh distributed. The Rayleigh density function is characterized by the PDF given in Eq. (2.29) [11].

$$
p_{r}(r)= \begin{cases}\frac{r}{\sigma^{2}} e^{\left(\frac{-r^{2}}{2 \sigma^{2}}\right)} & r \geq 0 \\ 0 & r<0\end{cases}
$$


$\mathrm{R} \sim \operatorname{Rayleigh}(\sigma)$ is a Rayleigh distribution if $R=\sqrt{X^{2}+Y^{2}}$ where $X$ and $Y$ are both $\sim N\left(0, \sigma^{2}\right)$, and are two independent normal distributions.

\section{Rayleigh Fading}

In practice, we encounter channels whose transmission characteristics vary with time. The time variations of the channel properties arise because of semi-periodic and random changes in the propagation characteristics of the medium. Hence, the effective channcl transfer function varies semi-periodically and randomly, causing random attenuation of the signal. This phenomenon is known as fading [11].

Raylcigh fading is a statistical model for the effect of a propagation environment on a radio signal, such as that used by wireless devices. It assumes that the power of a signal that has passed through such a transmission medium (also called a communications channel) will vary randomly, or fade, according to a Rayleigh distribution. It is a reasonable model for tropospheric and ionospheric signal propagation as well as the effect of heavily built-up urban environments on radio signals. Rayleigh fading is most applicable when there is no line of sight between the transmitter and receiver. If there is a line of sight, Rician fading is more applicable. In other words, Rayleigh fading is a reasonable model when there are many objects in the environment that scatter the radio signal before it arrives at the receiver. The requirement that there will be many scatterers present means that Rayleigh fading can be a useful model in heavily built-up city centers where there is no line of sight between the transmitter and receiver and many buildings and other objects attenuate, reflect, refract and diffract the signal.

In order to observe how Rayleigh fading is affecting the transmitted signal, two real C/A code were simulated and passed through the Rayleigh channel as illustrated in Fig. 2.8. In Fig. 2.8(a), the darker color represents the transmitted signal, and the lighter color shows the received signal. The resulting bit error rate (BER), is plotted and shown in Fig. 2.9. The lower curve in Fig. 2.9 represents the BER of the transmitted signal before fading. As expected, the received signal (after fading) has a higher BER due to the distortions. To 


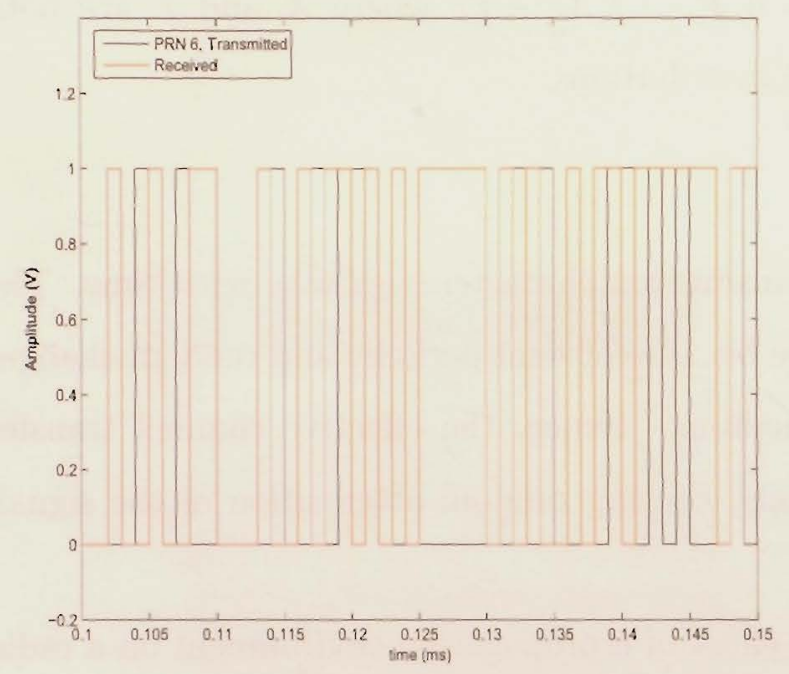

(a) PRN/CA Code Number 6 .

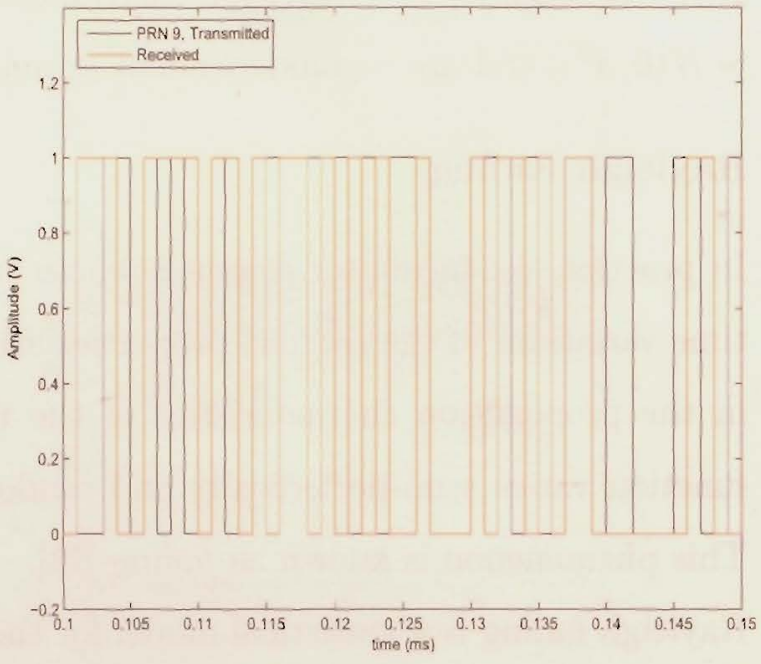

(b) PRN/CA Code Number 9.

Figure 2.8: The Effect of Rayleigh Channel on the GPS Signals.

generate Fig. 2.9, the author used a sequence of binary numbers passing through the Rayleigh channel (using "rayleighchan" Matlab function) without any signal combining techniques. This is just to illustrate how multipath could degrade the BER.

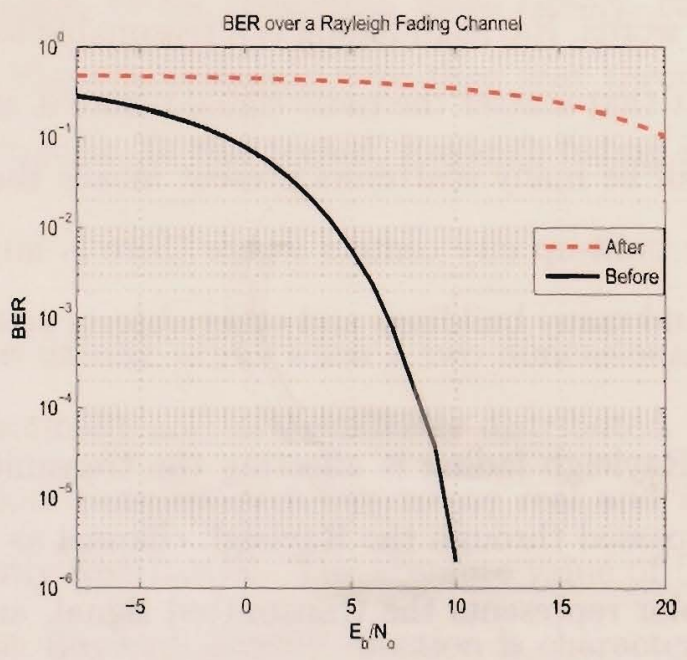

Figure 2.9: Bit Error Rate of Rayleigh Channel. 


\subsection{Current Multipath Mitigation Methods}

Currently, various mitigation techniques are available that help to minimize the effect of the errors contributing in position computations in the GPS world. Depending on the nature of applications, any of the following techniques could be applied. Generally, multipath mitigation could be categorized into two methods of hardware and software mitigation techniques. In terms of hardware improvements, antenna based mitigation methods, which are based on improving the antenna gain pattern by choke rings, are very common. The elimination of multipath signals is possible by selecting an antenna that takes advantage of the signal polarization. The transmitted GPS signals are right handed circularly polarized, whereas the reflected signals are left handed polarized. A choke ring consists of several concentric thin walls, or rings, around the center where the antenna element is located. These rings have no effect on direct signal. but from the reflected signals the rings create two so-called primary and secondary waves. The objective of the choke ring is for the primary and secondary reflected signals to substantially cancel each other, and the direct signal remains as the clominant signal. Using narrow correlator spacing and extending the multipath estimation delay lock loop is another approach to recluce the effect of multipath.

In terms of software mitigation. signal and data processing have been used by many researchers. Exploring the signal-to-noise ration, using multiple reference stations. smoothing carricr phase, and applying different filters are just a few to name.

Software mitigation has been improving so that many researchers have changed their approaches from the traditional denoising schemes, which are mainly based on Wiener filtering, to non-linear methods, especially those based on wavelets have become increasingly popular. The application of wavelets in signal processing especially in the area of global navigation satellite system (GNSS) has been of interest for many years. Wavelets are applied to the GNSS signal (i.e., GPS), to denoise and mitigate multipath errors which in turn helps to decrease the standard deviation of the computed positions. In the next section, some of the related work done by other scholars on wavelet analysis are discussed. 


\subsection{Related Work}

The use of wavelet analysis on GPS signals has proved to be an effective way of mitigating multipath. Satirapod and Rizos [12] applied wavelet decomposition technique on doubledifferenced residuals to extract multipath signals from GPS observations using data collected by three dual frequency receivers. Their approach would not be applicable on data collected from single antenna receivers (such as data used in this thesis), since there is no second antenna to form the double-differenced signal. Furthermore, in their experiment setup, they had access to a station which was considered to be multipath free (open sky), while in our case there was no open sky data available. In [12], the majority of the multipaths clata came from a concrete wall close to the second receiver, which could be considered as a fixed multipath. Whereas, the collected data for this thesis work is from street levels as opposed to [12] where their experiment was carried out on top of a building. In street level, the kind of multipath a receiver receives varies with time while it is constant on a roof.

Zhang and Bartone [13] applied both wavelet filtering and Wavesmooth techniques to mitigate multipath on dual frequency measurements as well. They used L1 and L2 frequencies to form ionosphere free code and carrier phase measurements, and then applied adaptive wavelet filtering on ion-free data. Their approach is also not useful when using a receiver which is not capable of receiving L2-band data, which is the case for almost all the inexpensive receivers (at the time of writing the thesis).

Xia and Liu [14] applied wavelet transform to double differential phase observations to mitigate phase multipath in GPS relative positioning. Applying their method also requirs access to the L2-band data. Furthermore, in all the cases mentioned above ([12]-[14]), there is no indication of threshold selection and how the threshold value could affect the results. The value of the threshold has a significant impact on the signal smoothing process.

In [12]-[14], there is no actual positioning after multipath extraction. While goal of multipath reduction is to increase the position precision. Whereas, in this thesis the experiment was carried out based on actual positioning, and at the end the degradation or improvement are clearly expressed in terms of some statistical quantities such as standard deviation, Kurtosis, 
and skewness of the positions. In this thesis, wavelet analysis technique is applied to a set of clata taken from a single stationary receiver operating on $L 1$ frequency. Since the focus of this thesis is on multipath mitigation by means of multi-resolutional analysis, the next chapter gives an overview on wavelets. 


\section{Chapter 3}

\section{Wavelet Analysis of GPS Signal}

\subsection{Wavelet Analysis Overview}

Although wavelet analysis goes back to 1930s, the development of the technique for various applications in engineering and the sciences began in 1980s. In that relatively short time, the wavelet analysis has been adopted to a variety of applications, from fingerprint compression, coding (audio and video), and classification to improved processing of GPS data [15].

Almost all the signals gencrated by pieces of equipment such as receivers carry some kind of noises, or they are biased by multipath in their measurement. One of the best tool available to process the data is wavelet. The fundamental behind wavelet analysis is to break up the signal into different frequencies and investigate each component in terms of its scale.

As oppose to sinusoidal waves, which are considered to be big waves(infinite cluration), wavelets are small waves. In other words, wavelets are finite in cluration. Where sinusoids are smooth and predictable, wavelets tend to be irregular and asymmetric. Fig. 3.1 shows

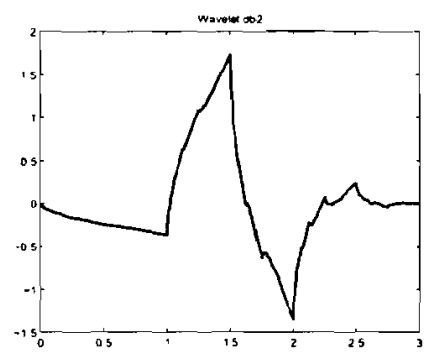

Figure 3.1: A Typical Wavclet (db2). 
a typical wavelet known as $\mathrm{db} 2$. Wavelets are analysis tools mainly used for image or time series analysis. $\psi(t)$ is considered to be a wavelet function if the following conditions are met $[16]$.

$$
\begin{aligned}
& \int_{-\infty}^{\infty} \psi(t) d t=0 \\
& \int_{-\infty}^{\infty} \psi^{2}(t) d t=1
\end{aligned}
$$

Eq. (3.1) tell us that the average value of the wavelet has to be zero, and Eq. ( 3.2) represent unit energy property of the wavelets.

As Fourier analysis is to break up a signal into sine waves of various frequencies, wavelet analysis breaks up a signal into shifted and scaled versions of the wavelet.

Generally speaking there are two kinds of wavelets.

1. Continuous wavelet transform (CVT)

2. Discrete wavelet transform (DWT)

\section{Continuous wavelet transform (CWT)}

Wavelet transform is a tool that cuts up data or function into different frequency components, and then studies each component with a resolution matched to its scale [17]. In signal analysis, the wavelet transform allows us to view a time history in terms of its frequency components. In this respect the wavelet transform performs in much the same way as the Fourier transform [18]. The Fourier transform extracts from the signal details of the

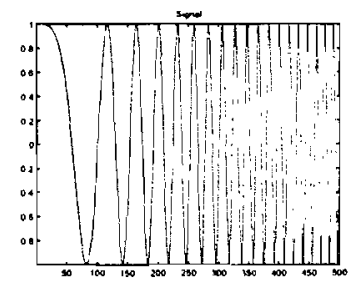

(a) Raw Signal

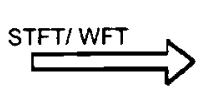

(b) Fixed Tiling

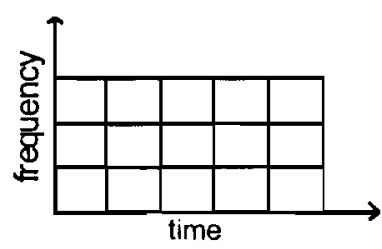

Figure 3.2: Constant Resolution of a Windowed Signal (STFT). 
frequency content but loses all information on the location of a particular frequency within the signal. Time localization must then be achieved by first windowing the signal, and then by taking its Fourier transform. The problem with windowing is that the slice of the signal that is extracted is always the same length. Thus, the time slice (number of data points) used to resolve a high frequency component is the same as the number used to resolve a low frequency component [18]. Fig. 3.2 shows the constant resolution of the windowed signal. The window Fourier transform (WFT), also known as short time Fourier transform (STFT), of a time function $f(t)$ is defined as $[16]$ :

$$
S_{f}(t, w)=\int h\left(t-t_{1}\right) f\left(t_{1}\right) e^{-j w t_{1}} d t_{1}
$$

where $h(t)$ is a given window function satisfying $0<\int|h(t)|^{2} d t<\infty$. In practice, the window function $h(t)$ is usually a lowpass function [16]. The Short-Time Fourier Transform (STFT), maps a signal into a two-dimensional function of time and frequency as shown in Fig. 3.2. The STFT represents a sort of compromise between the time and frequency based

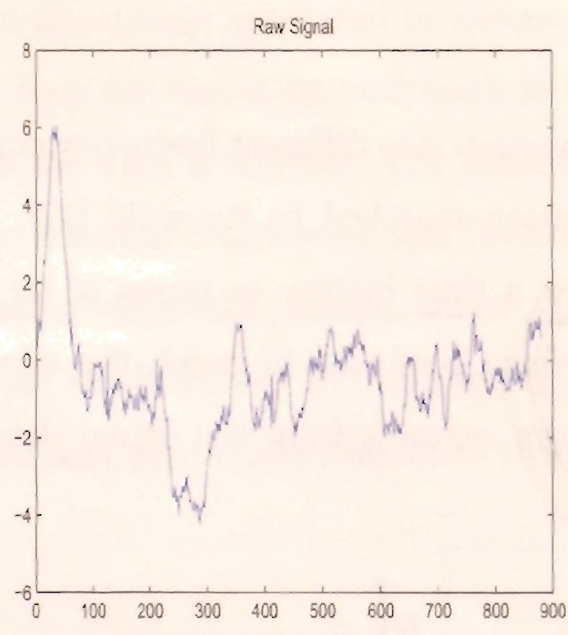

(a) Real value GPS Residual Signal

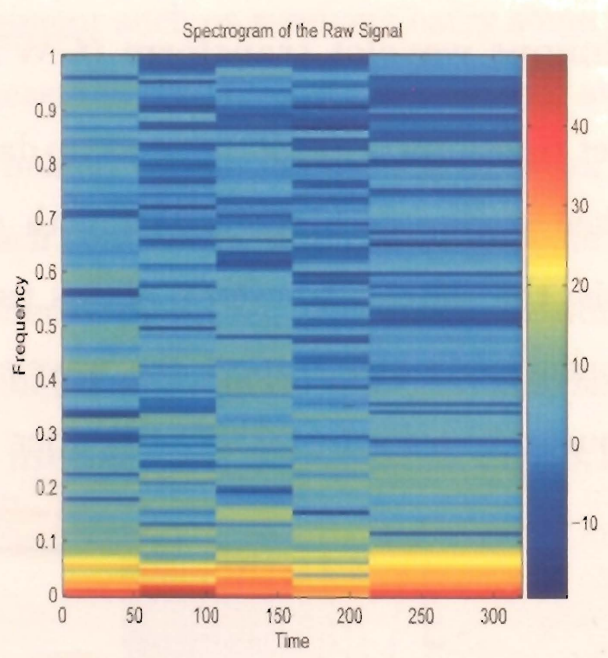

(b) Spectrogram of the Signal

Figure 3.3: The Windowed Fourier Transform of the Raw Signal Using a Sliding Window.

views of a signal. It provides some information about both when and at what frequencies a signal event occurs. However, one can only obtain this information with limited precision, 
and that precision is determined by the size of the window as shown in Fig. 3.3. A short window will produce an excellent time resolution, but a poor frequency resolution. A long window will produce the opposite.

In contrast to windowed Fourier transform, the wavelet adapts the width of its time-slice according to the frequency components being extracted. In other words, the resolution is high for high frequency components and low for low frequency components, because wavelet uses short windows at high frequency and long window at low frequency as shown in Fig. 3.4. In wavelet analysis the scale that the data is looked at plays a special role. Wavelet

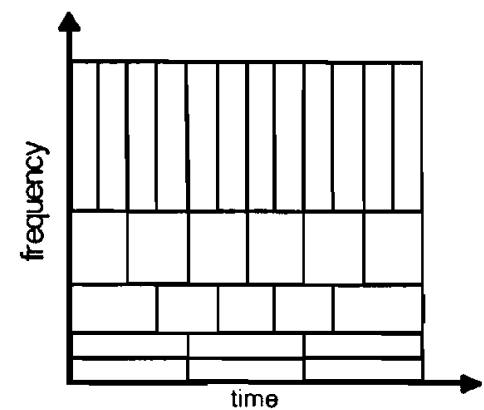

Figure 3.4: One Representation of a Time-Frequency Tile for the Wavelet Transform.

algorithm process data at different scales or resolutions. If one looks at a signal with a large window, the gross features will be noticed. Similarly, if one looks at a signal with a small window, the small features will be noticed [19].

According to the definition of the inner product, the CWT can be thought of as an inner product of the original signal with scaled, shifted versions of the basis wavelet function $\psi(t)$ $[15]$,

$$
<x, \psi_{a, b}>=C W T(a, b)=\int x(t) \psi(t) d t
$$

where:

$$
\psi_{a, b}=\frac{1}{\sqrt{a}} \psi\left(\frac{t-b}{a}\right)
$$

In which "a" represents the scale (dilation) and " $\mathrm{b}$ " is the time-shift (translation) parameter. Therefore, the wavelet transform of a continuous (analog) signal $x(t)$ is known as CWT which 
is defined as:

$$
C W T(a, b)=\frac{1}{\sqrt{a}} \int_{-\infty}^{\infty} x(t) \psi\left(\frac{t-b}{a}\right) d t
$$

Fig. 3.5 shows the scalogram (CWT) of the signal with the scales from 1 to 5 . The wavelet

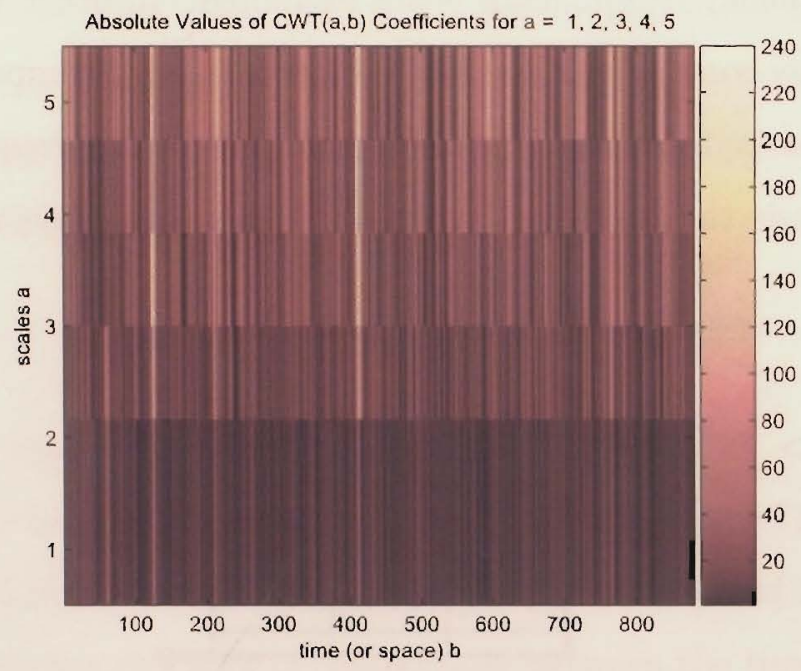

Figure 3.5: Scalogram of the Signal.

functions have time-widths adapted to their frequency such that high frequency are narrow, while low frequency are much wider. As a result, the wavelet transform is better to "zoom in" on very short lived high frequency phenomenon, such as transients [17].

\subsubsection{Multilevel Representation of a Function}

In order to develop a multilevel representation of a function in $L^{2}(R)$, a sequence of embedded subspaces $\boldsymbol{V}_{\boldsymbol{i}}$ is needed such that [18]:

$$
\ldots \subset \mathrm{V}_{-1} \subset \mathrm{V}_{0} \subset \mathrm{V}_{1} \subset \mathrm{V}_{2} \subset \ldots \subset \mathrm{L}^{2}(\mathrm{R})
$$

with the following properties:

- The embedded subspaces are related by a scaling law

$$
g(x) \in \mathbf{V}_{\mathbf{j}} \Leftrightarrow \mathbf{g}(2 \mathbf{x}) \in \mathbf{V}_{\mathbf{j}+1}
$$


- Each subspace is spanned by integer translates of a single function $g(x)$ such that:

$$
g(x) \in \mathbf{V}_{\mathbf{0}} \Leftrightarrow \mathbf{g}(\mathbf{x}+\mathbf{1}) \in \mathbf{V}_{\mathbf{0}}
$$

Therefore, a scaling function $\phi(x) \in V_{\mathbf{0}}$ is needed such that its integer translates $\{\phi(x-k), k \in Z\}$ form a Riesz basis for the space $V_{0}$. Then $\phi(2 x-k)$ form a basis for the space $V_{1}$. Thus:

$$
\begin{array}{r}
\mathrm{V}_{0}=\operatorname{span}\{\phi(\mathrm{x}-\mathrm{k}), \mathrm{k} \in \mathrm{Z}\} \\
\mathrm{V}_{\mathbf{1}}=\operatorname{span}\{\phi(2 \mathrm{x}-\mathrm{k}), \mathrm{k} \in \mathrm{Z}\}
\end{array}
$$

Since the space $\boldsymbol{V}_{\mathbf{0}}$ lies within the space $\boldsymbol{V}_{\mathbf{1}}$, then $\boldsymbol{V}_{\mathbf{0}}$ can be expressed in terms of the basis functions of $V_{1}$ :

$$
\phi(x)=\sum_{k=-\infty}^{\infty} a_{k} \phi(2 x-k)
$$

Eq. (3.12) is known as dilation or scaling function. The constant coefficients $a_{k}$ are called filter coefficients, and it is often the case that only a finite number of these are non-zero [18]. The filter coefficients are derived by imposing certain conditions on the scaling function. One of these conditions is that scaling function and its translations should form an orthonormal set i.e.:

$$
\begin{gathered}
\int_{-\infty}^{\infty} \phi(x) \phi(x+l) d x=\delta_{0, l} \quad l \in Z \\
\delta_{0, l}= \begin{cases}1 & \mathrm{l}=0 \\
0 & \text { otherwise }\end{cases}
\end{gathered}
$$

A wavelet, $\psi(x)$, is orthogonal to the scaling function, $\phi(x)$, and is defined by:

$$
\psi(x)=\sum_{k=-\infty}^{\infty}(-1)^{k} a_{N-1-k} \phi(2 x-k)
$$

where $N$ is an even integer. This definition satisfies orthogonality since from Eqs.(3.12) and (3.14) we have:

$$
\begin{array}{r}
\langle\phi(x), \psi(x)\rangle=\int_{-\infty}^{\infty} \sum_{k=-\infty}^{\infty} a_{k} \phi(2 x-k) \sum_{l=-\infty}^{\infty}(-1)^{l} a_{N-1-l} \phi(2 x-l) d x \\
\Rightarrow\langle\phi(x), \psi(x)\rangle=\frac{1}{2} \sum_{k=-\infty}^{\infty}(-1)^{k} a_{k} a_{N-1-k}=0
\end{array}
$$


The sets of coefficients $\left\{a_{k}\right\}$ and $\left\{(-1)^{k} a_{N-1-k}\right\}$ are said to form a pair of quadrature mirror filter [18]. Fig. 3.6 shows Daubechies (db2) scaling function and its corresponding wavelet.

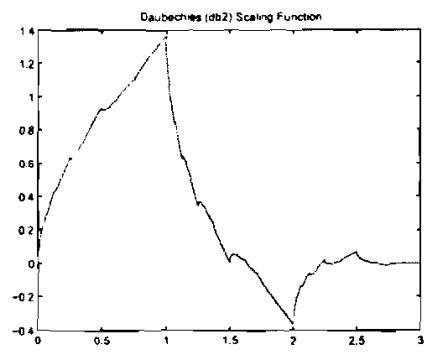

(a) db2 Scaling Function.

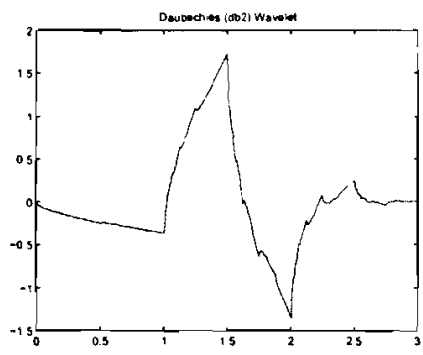

(b) db2 Wavelet Function

Figure 3.6: Daubechics (db2) Scaling and Corresponding Wavelet Functions.

\subsection{Discrete Wavelet Transform (DWT)}

The time series signals are sampled and discretized in case we are working with CWT. In other words, the wavelet series are simply a sampled version of the CWT, and the amount of information it provides is highly redundant as far as the reconstruction of the original signal is concerned. This redundancy requires a significant amount of time and memory. On the other hand, DWT provides sufficient information both for analysis and synthesis of the original data with a significant reduction in time and memory. DWT can provide filter bank structure which is amicable for hardware implementation. In DWT, the scaling and shifting variables are discretized so that wavelet coefficients can be described by two integers, $m$ and $n$ [15]. Thus, the DWT is given as:

$$
D W T(m, n)=\frac{1}{\sqrt{a_{0}^{m}}} \sum_{k} x[k] \psi\left[a_{0}^{-m} n-k\right]
$$

where $x[k]$ is a signal or a digitized version of an analog signal with sample index $k$, and $\psi[n]$ is the wavelet. With different choices of $m$ we obtain a geometric scaling: $1,1 / a_{0}, 1 / a_{0}^{2}, \ldots$. It is found in practice that the most convenient value of $a_{0}$ is 2 . This analysis method then consists of decomposing a signal into components at several frequency levels that are related 
by powers of two (a dyadic scale) [15].

Let $\boldsymbol{X}$ be the observed time series and for convenience assume $N$ is integer multiple of $2^{j}$.

$$
X=\left[X_{0}, X_{1}, \ldots, X_{N-1}\right]^{T}
$$

Let $\boldsymbol{w}$ be $N \times N$ orthonormal DWT matrix and $\boldsymbol{W}$ be the vector of DWT coefficients [16].

$$
\begin{gathered}
W=w X \Rightarrow X=w^{T} W \\
X \Leftrightarrow W
\end{gathered}
$$

The DWT can be looked at as the multiresolutional decomposition of a sequence. It takes

a length $\mathrm{N}$ sequence $\boldsymbol{x}(\boldsymbol{n})$ as input and generates a length $\mathrm{N}$ sequence as the output. The output has $\mathrm{N} / 2$ values at the highest resolution and $\mathrm{N} / 4$ values at the next resolution, and so on, that is the frequency resolution is low at the high frequencies and high at the low frequencies, whereas the time resolution is high at the higher frequencies and low at the lower frequencies.

\subsection{Filter Banks}

The idea is the same as it is in DWT. A time-scale representation of a digital signal is obtained using digital filtering techniques. To process the data, we look into the low and high frequency contents of the signal. In case of working with stationary data, the Fourier analysis is sufficient, whereas working with a time varying data the Fourier analysis won't be a suitable tool since Fourier is not able to show the time localization. Fourier basis functions are localized in frequency but not in time. Small frequency changes in the Fourier transform will produce changes everywhere in the time domain. Wavelets are local in both frequency (scale) via dilations and in time via translation. This localization makes wavelet transform a suitable tool for time varying signals. It is well known that the computational complexity of the fast Fourier transform is $\boldsymbol{O}\left(n \log _{2}(n)\right)$, while for discrete wavelet transform the computational complexity goes down to $O(n)$ [21].

For many signals, the low frequency content is the most important part. Considering the 
human voice, if one removes the high frequency components, the voice sounds different, but you can still tell what's being said. However, if you remove enough of the low frequency components, you hear gibberish. In wavelet analysis we often speak of approximation and details. The approximations are the high-scale, low frequency components of the signal. The details are the low-scale, high frequency components [22]. Fig. 3.7 shows a typical data analysis by wavelet.

raw data $\rightarrow$ wavclet decomposition $\rightarrow$ thresholding $\rightarrow$ wavclct rcconstruction $\rightarrow$ filtered data

Figure 3.7: A Typical Block Diagram of Wavclet Analysis.

\section{Filtering Process}

The data with coarse resolution contains information about lower-frequency components and retains the main features of the original signal. The data with finer resolution retains information about the higher-frequency components. The filtering approach to multi-resolutional wavelet transform is to form a series of half band filters that divide a spectrum into a high frequency band and a low frequency band. It is formulated on a scaling function or low-pass filter and a wavelet function or high-pass filter. Multiresolutional analysis builds a pyramidal structure that requires an iterative application of scaling and wavelet functions to lowpass and highpass filters, respectively [15].

Theses filters initially act on the entire signal band at the high frequencies (small scale) first and gradually reduce the signal band at each stage as shown in Fig. 3.8. Therefore, filtering process begin with passing the raw data through a few high-pass filters $\left(\boldsymbol{h}_{\boldsymbol{l}}\right)$ and their complementary low-pass filters $\left(g_{l}\right)$. Fig. 3.8 shows a three level wavelet decomposition. First the raw data passes through a high and low pass filter (here filters of db2 type). This decomposition divides the raw signal into level one approximation (a1) and detail (d1). Then in turn level one approximation will be passed through the same filtering to construct the level two approximation (a2) and detail (d2). This process can be iterated to a suitable 


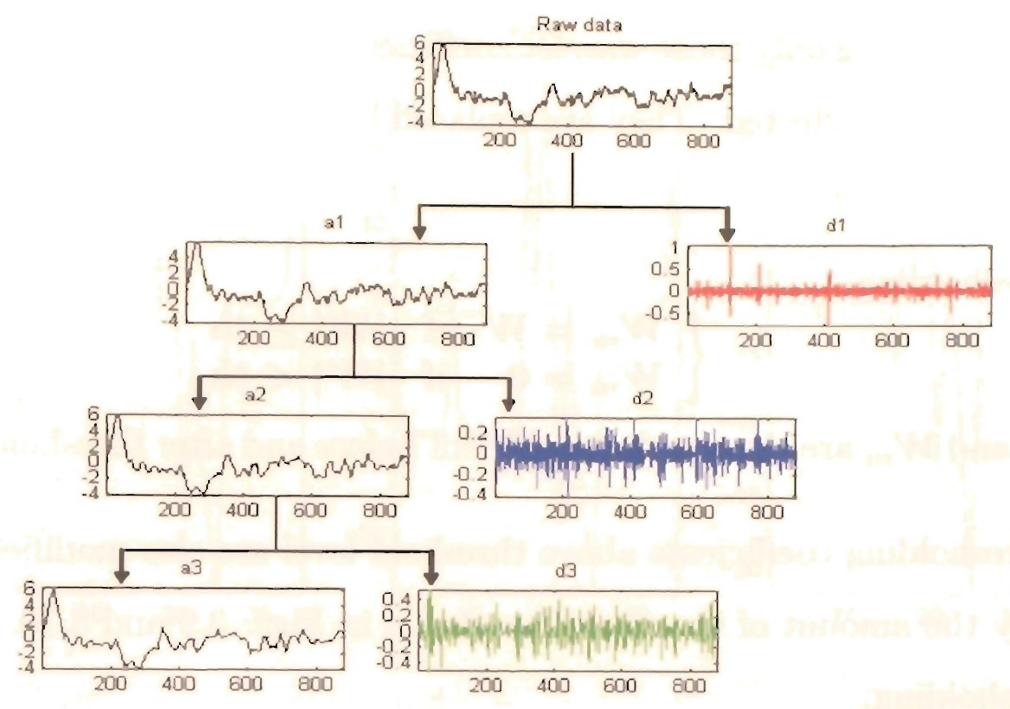

Figure 3.8: A Three level Wavelet Decomposition.

number of levels based on the nature of the signal. The signal re-construction, in this case, will be made from the last level of approximation (a3) plus the detail levels.

$$
\text { Reconstructed Signal }=a 3+d 3+d 2+d 1
$$

\subsection{De-noising}

In wavelet decomposition when details are small, they might be omitted without substantially affecting the original signal. Thus, the idea of thresholding wavelet coefficients is a way of cleaning out "unimportant" details considered to be noise [21]. Therefore, denoising refers to manipulation of wavelet coefficients for noise reduction in which coefficient values below a carefully selected threshold level are replaced by zero after which an inverse transform of modified coefficients is used to recover denoised signal. Mathematically, thresholding can also be described by a transformation of the wavelet coefficients in which transform matrix is a diagonal matrix with elements 0 or 1 [23]. Zero elements forces the corresponding coefficient below a given threshold to be set to zero while others corresponding to one, reduces the coefficients by the given threshold.

The two approaches which are considered for denoising are hard and soft thresholding. 
- In hard thresholding only those wavelet coefficients with absolute values below or at the threshold level are affected. They are replaced by zero and others are kept unchanged as shown in Fig. 3.9 .

Hard Thresholding:

$$
\begin{cases}W_{m}=W & \text { if } \quad|W| \geq t h \\ W_{m}=0 & \text { if } \quad|W|<t h\end{cases}
$$

where $\boldsymbol{W}$ and $\boldsymbol{W}_{m}$ are the wavelet coefficient before and after thresholding respectively.

- In soft thresholding coefficients above threshold level are also modified where they are reduced by the amount of threshold ${ }^{1}$ as shown in Figs. 3.9 and 3.10 .

Soft Thresholding:

$$
\left\{\begin{array}{lll}
W_{m}=\operatorname{sign}(W)(|W|-t h) & \text { if } & |W| \geq t h \\
W_{m}=0 & \text { if } & |W|<t h
\end{array}\right.
$$
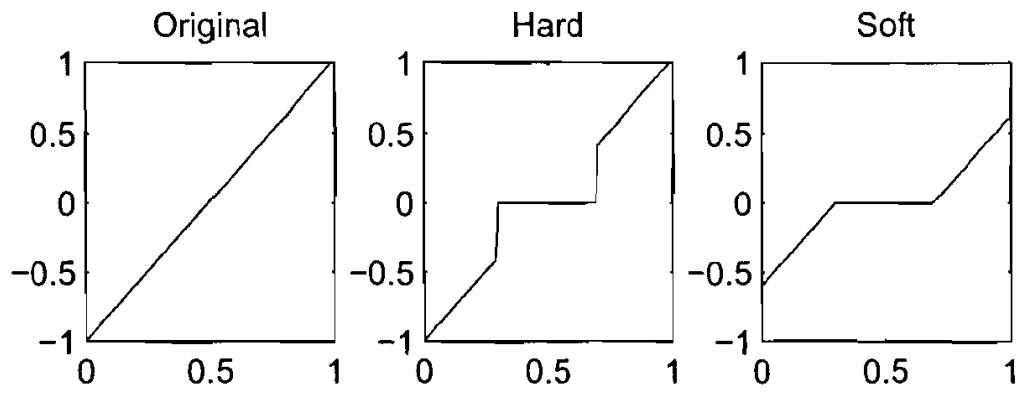

Figure 3.9: Illustration of Soft and Hard Thresholding

\subsubsection{Noise Reduction Model}

A basic model used to analysis the noise content of a signal is shown in Eq. (3.18).

$$
X(k)=S(k)+\sigma N(k), \quad k=1,2, \ldots, n
$$

In Eq. (3.18), $\boldsymbol{X}(\boldsymbol{k})$ is the measurements (known to us), $\boldsymbol{S ( k )}$ is the true values of the signal (unknown), $\sigma$ is the noise level (unknown), $N(k)$ is the noise components (unknown), and

\footnotetext{
${ }^{1}$ Donoho refers to soft thresholding as 'shrinkage' since it can be proved that reduction in coefficient amplitudes by soft thresholding, also results in a reduction of the signal level thus a 'shrinkage' [23].
} 

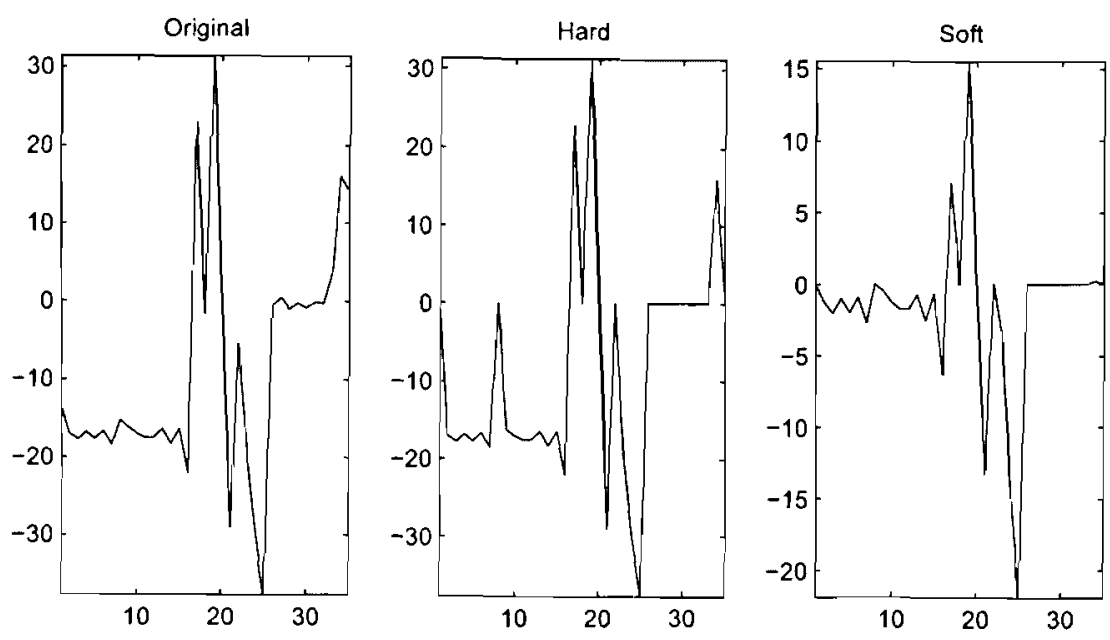

Figure 3.10: Soft and Hard Thresholding of the real GPS Data

$\boldsymbol{n}$ is the signal length. Under an orthogonal decomposition, each coefficient is decomposed into component attributed to the true signal values, $s(n)$, and to noise as follows [23]:

$$
c_{i}=u_{i}+d_{i}, \quad i=1,2, \ldots, n
$$

or in a vector form

$$
C=U+D
$$

where $\boldsymbol{C}, \boldsymbol{U}$, and $\boldsymbol{D}$ are the vector representation of empirical (observed) wavelet coefficients, true coefficient values and noise content of the coefficients respectively. It is assumed that coefficients at the high frequency details provicle a good estimate of the noise content of the signal [23].

\section{Standard Deviation and Threshold Level Estimation}

In noise reduction under Gaussian white noise, the estimation of standard deviation $\sigma$, in Eq. (3.18), is used to determine a suitable threshold. An estimate of the standard deviation at the $\boldsymbol{d} \mathbf{1}$ level (details at the level one coefficients) is then used to determine a suitable threshold level for coefficient thresholding at all levels [23]. This approach is global thresholding which is applied to all detail coefficients. The justification for this approach 
is that at the high frequency $\boldsymbol{d} 1$ level, only a few large amplitude coefficients carry much of the signal information and a large portion of the coefficients attribute to insignificant information and considered to be noise. In case of non-white (colored) noise, we still impose white noise model on the coefficients, however, threshold levels are considered to be level (scale) dependent [23]. Gaussian white noise model is used on detail coefficients where standard deviation and threshold levels are evaluated for each level separately.

\subsubsection{Threshold Selection}

The quality of the signal estimation is investigated by the risk function. A risk function (or loss function) is mainly the variance of the estimation error, i.e. the difference between the estimated value and the actual unknown value. The risk function is defincd as follows:

$$
R(S, \hat{S})=E\|(S-\hat{S})\|^{2}
$$

where $\boldsymbol{S}$ and $\hat{\boldsymbol{S}}$ are the actual and the estimated values of a signal respectively. Minimizing the risk function results in an estimate of the variance of the signal [23]. Since the noise component is assumed to be zero mean Gaussian, the difference $(\boldsymbol{S}-\hat{\boldsymbol{S}})$ is a measure of an error. Therefore, Eq. (3.19) is a measure of the energy of the noise, i.e. $\sum[N(k)]^{2}$. One best estimate is obtained using minimax rule $[23,24]$,

$$
\operatorname{minmax} R(S, \hat{S})=i n f_{s} \sup (S-\hat{S})
$$

where inf and sup represent the infimum and supremum respectively. In analysis the infimum or greatest lower bound of a set $S$ of real rumbers is denoted by $\inf (S)$ and is defined to be the biggest real number that is smaller than or equal to every number in $\mathrm{S}$. The supremum or least upper bound of a set $\mathrm{S}$ of real numbers is denoted by $\sup (\mathrm{S})$ and is defined to be the smallest real number that is greater than or equal to every number in $\mathrm{S}$.

Based on orthogonal decomposition, minimization of the risk function at the signal level as defined by Eq. (3.19), can equivalently be defined at the cocfficient level as shown below $[23]:$

$$
R(S, \hat{S})=E\|S-\hat{S}\|^{2}=E\|C-\hat{C}\|^{2}
$$


where $\hat{C}$ is the estimate of the true coefficient values. Accordingly, minimization of the risk function at the coefficient level results equivalently in estimating the true vale of the signal. Under the assumption of i.i.d. for the wavelet coefficients and Gaussian white noise, [24] obtained a solution which minimizes the maximum risk,

$$
t h=\frac{\sigma}{\sqrt{n}} \sqrt{2 \log (n)}
$$

where th is the threshold value, and $\mathrm{n}$ is the length of the signal. In Donoho's estimator, Eq. (3.22), the only unknown is the value of the $\boldsymbol{\sigma}$. Therefore, estimation of the $\boldsymbol{\sigma}$ in Eq. (3.22) is the main challenge when using it.

There are two general methods for estimating the $\sigma$ in Eq. (3.22). As mentioned in section 3.4.1, the standard deviation at the $\mathrm{d} 1$ level (details at the level one coefficients) is used to determine a suitable threshold level for coefficient thresholding at all levels. This approach is global thresholding which is applied to all detail coefficients.

The second method to estimate the standard deviation, $\sigma$, is to apply Eq. (3.23) [23],

$$
\sigma^{2}=\frac{\text { median }(|c|)}{0.6745}
$$

where $c$ is the wavelet coefficients. For level dependent thresholding, thresholds are rescaled at each level to arrive at a new estimate corresponding to the standard deviation of wavelet coefficients at that level.

\subsection{Conventional Thresholding}

So far the value of the threshold was based on an estimation value which in turn depends heavily on the standard deviation $\sigma$. The noise level could change with time as it depends on the channel. For example in case of GPS, most of the received signals contain multipaths which could be caused due to any obstructions. Fig. 3.11 shows a typical real GPS data.

Figure 3.12 illustrates the soft and hard thresholding based on Donoho (Eq. (3.22)). Fig. 3.12(a) was plotted based on the noise level of the $d 1$ coefficients, while Fig. 3.12(b) 


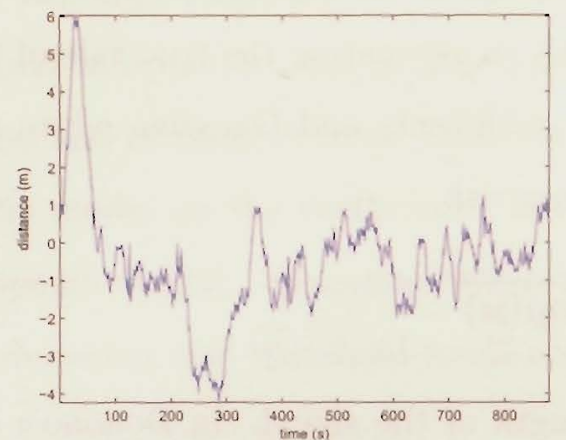

(a) A Sample of Good Data.

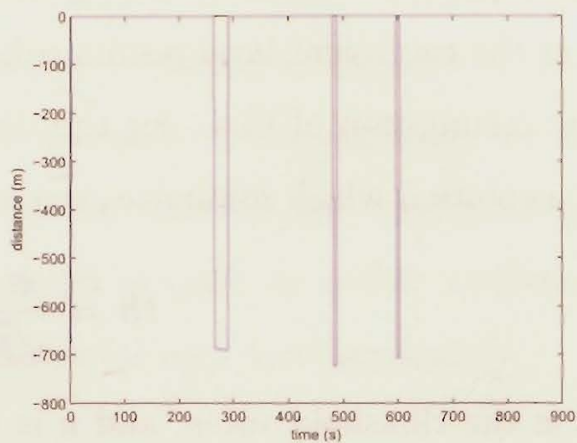

(b) A Sample of Bad Data

Figure 3.11: A Typical Residual Data to Be Analyzed.

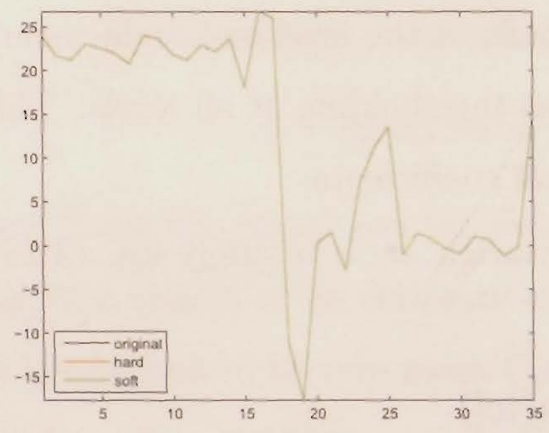

(a) Thresholding the Good Data, Based on $\operatorname{std}(d 1)$.

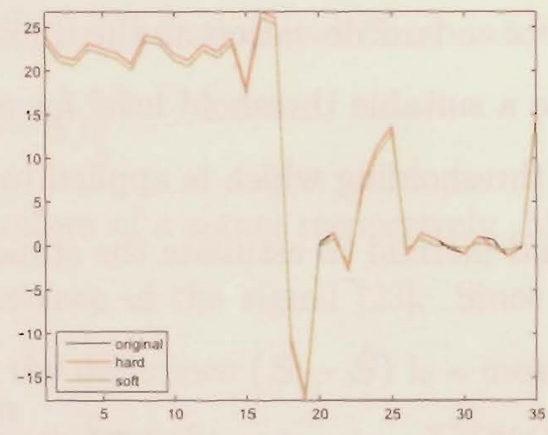

(b) Thresholding the Good Data, Based on Eq. (3.23).

Figure 3.12: Thresholding the Wavelet Coefficients Based on Donoho's Equation.

was plotted based on the $\mathrm{Eq}(3.23)$. A simple visual inspection confirms that there is no significant difference between Fig3.12(a) and Fig3.12(b). In both cases since the noise levels were low, the difference between the original and the thresholded coefficients are almost zero. In some signal processing such as GPS data, the repetition of sudden changes of the data especially from zero to a high value (usually above $200(\mathrm{~m})$ ), which acts like spike, is an indication of error in measurement as shown in Fig. 3.11(b). The threshold value obtained by [24] won't be suitable for such data. In other words, we need a criteria that sets a limit for data to be used. As shown in Fig. 3.14, in processing a data biased by high multipath values, the reconstructed signal has artifacts when applying Donoho's estimator, which in 


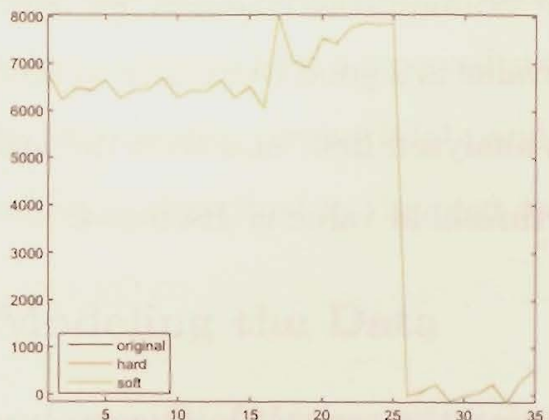

(a) Thresholding the Bad Data, Based on std(d1).

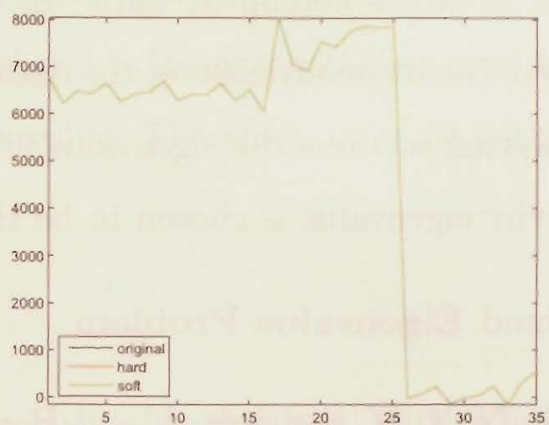

(b) Thresholding the Bad Data, Based on Eq. (3.23).

Figure 3.13: Thresholding the Wavelet Coefficients Based on Donoho's Equation.

turn creates wrong results. In the next section we introduce a new threshold value based on

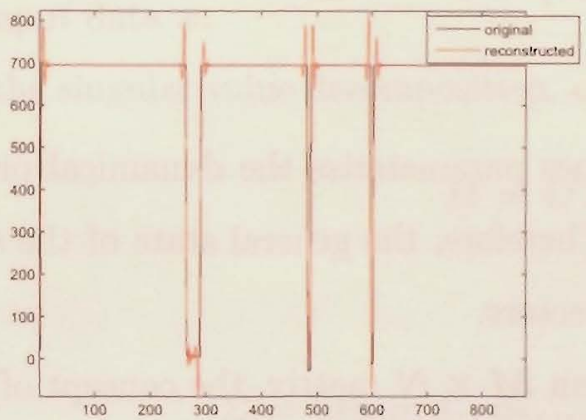

(a) Reconstruction of the data, level-2, db4.

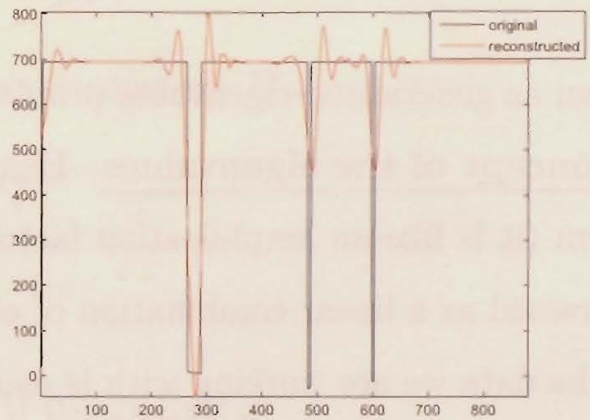

(b) Reconstruction of the data, level-4, db7.

Figure 3.14: Wavelet Analysis of a GPS Data.

eigenvalue which will enable us to investigate the signal more closely.

\subsection{The Proposed Thresholding Method}

We are looking for an upper-bound threshold value that ignores the high sharp changes in a data set and reduces the coefficients by some amounts that represents the effect of the biased values, since the effect of multipath could be seen as time delay or extra distances that the signal goes through. Therefore, the added distances have to be estimated and consequently 
subtracted from the corrupted data. In order to estimate the changes, we need a criteria which shows the dispersiveness of the data. Eigenvalue is a good candidate to be considered. In the following sections the eigenvalue criteria is analyzed first, and then the answer to the question why eigenvalue is chosen to be the new threshold value is discussed.

\section{Generalized Eigenvalue Problem}

Given two $N \times N$ matrices $\mathrm{A}$ and $\mathrm{B}$, determining the scalars $\boldsymbol{\lambda}_{\boldsymbol{i}}$ (eigenvalues) and $\boldsymbol{X}_{\boldsymbol{i}}$ (eigenvectors) such that:

$$
A X_{i}=\lambda_{i} B X_{i}
$$

or equivalently determine the scalars $\boldsymbol{\lambda}_{\boldsymbol{i}}$ such that:

$$
\operatorname{det}\left(A-\lambda_{i} B\right)=0
$$

is known as generalized eigenvalue problem.

The concept of the eigenvalues: Eigenvalues parameterize the dynamical properties of a system (it is like an amplification factor). Therefore, the general state of the system can be expressed as a linear combination of eigenvectors.

Since the data we are working with is usually an $M \times N$ matrix, the concept of eigenvalue problem becomes a singular value decomposition issue.

\section{Singular Values Decomposition}

Suppose $\boldsymbol{D}$ is an $M \times N$ matrix, then there exists a factorization of the form:

$$
\boldsymbol{D}=\boldsymbol{U} \boldsymbol{\Sigma} V^{*}
$$

where $U$ is an $M \times M$ unitary matrix, $\boldsymbol{\Sigma}$ is an $M \times N$ matrix with nonnegative numbers on the diagonal and zeros off the diagonal, and $V^{*}$ denotes the conjugate transpose of $\boldsymbol{V}$, an $N \times N$ unitary matrix. Such a factorization is called a singular value decomposition of D.

The matrix $\boldsymbol{\Sigma}$ contains the singular values, which can be thought of as scalar gain control, 
by which each corresponding input is multiplied to give a corresponding output.

Now, in order to observe how a received signal is related to its eigenvalue we need to obtain a relationship between the received data and its eigenvalue. Therefore, we start with modeling the data, which is described in the next section.

\subsubsection{Modeling the Data}

We assume there exists $N$ transmitters and $M$ receivers (or channels) with a full $M \times N$ channel matrix $\boldsymbol{H}$ in between. Therefore, the data received at the $M$ elements can be modeled as:

$$
y=H x+n,
$$

where $M$ parallel channels are AWGN with a noise level of $\sigma^{2}$, and $y$ is the received data from the input data $x$.

Based on the singular value decomposition, one can decompose $\boldsymbol{H}$ as:

$$
H=U \Sigma V^{H}
$$

and,

$$
\Sigma=\left[\operatorname{diag}\left(d_{1}, d_{2}, \ldots, d_{M}\right) \mid 0_{M \times N-M}\right]
$$

where $U$, and $V$ are the eigenvectors, and $d_{m} \geq 0$ are the $M$ singular values (eigenvalues) of $\boldsymbol{H}$. By substituting Eq. (3.28) in Eq. (3.27),

$$
y=U \Sigma V^{H} x+n
$$

and multiplying both sides by $\boldsymbol{U}^{\boldsymbol{H}}$ :

$$
\begin{array}{r}
\Rightarrow U^{H} y=U^{H} U \Sigma V^{H} x+U^{H} n \\
\Rightarrow U^{H} U=I_{M} \\
\Rightarrow U^{H} y=\Sigma V^{H} x+U^{H} n \\
\Rightarrow \tilde{y}=\Sigma \tilde{x}+\tilde{n}
\end{array}
$$


where $\tilde{y}=U^{H} \boldsymbol{y}$ and $\tilde{x}=V^{H} x$.

Eq. (3.34) shows how the values of the transmitted signals could be affected by the eigenvalues. The matrix $\boldsymbol{\Sigma}$ can be thought of as scalar gain control, by which each corresponding input is multiplied to give a corresponding output.

Therefore, the eigenvalues in the received data are representing the extreme deviation and model the dispersiveness that exists among the data. Consequently, the eigenvalue is considered to be the upper-bound threshold value used in soft thresholding of the wavelet coefficients.

Soft Thresholding:

$$
\left\{\begin{array}{lll}
C_{m}=\operatorname{sgn}(C)(|C|-t h) & \text { if } \quad|C| \geq t h \\
C_{m}=0 & \text { if } \quad|C|<t h
\end{array}\right.
$$

where:

$$
\operatorname{sgn}(C)= \begin{cases}1 & C>0 \\ 0 & C=0 \\ -1 & C<0\end{cases}
$$

and

$$
t h=e i g(C)
$$

In the above equations $C_{m}$ represents the modified coefficients after filtering and $C$ represents the coefficients before thresholding. Fig. 3.15 shows the soft and hard thresholding of the coefficients based on the new threshold value. Clearly the difference between the hard and soft thresholding is distinguishable.

\subsection{Chapter Summary}

In this chapter wavelet analysis was investigated with the focus on a new thresholding system. Although the thresholding by Donoho's equation is a possibility, there are signals which Eq. (3.22) will not be applicable. The estimation of the noise level in Eq. (3.22) is a major draw back. The value of the noise level in data might be low but the bias value caused by multipath could be very high. In these cases the value of $\sigma$ will not be the correct value 


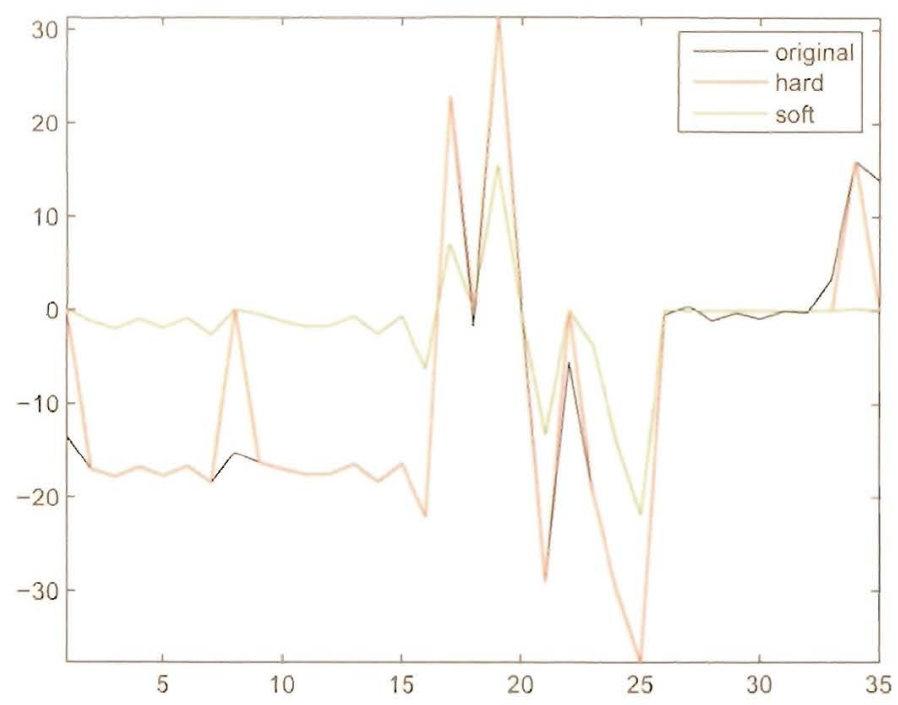

Figure 3.15: Soft and Hard Thresholding of the real GPS Data

to be chosen as a threshold value. In our study we proposed a new threshold value based on eigenvalue of the wavelet coefficients. Eigenvalue parameterizes the dynamical properties of a system (it is like an amplification factor). In other words, eigenvalue represents the extreme deviation and models the dispersiveness that exists among the data. This value is considered to be the upper-bound threshold value. 


\section{Chapter 4}

\section{Multipath Mitigation Strategies}

As we discussed previously in Chapter 2 (section 2.6), there are different methods which enable us to mitigate multipath. However, they are not suitable if a user is using a single frequency receiver. The work presented in this thesis is based on an autonomous and L1 frequency receiver. Therefore, we looked for another approach to reduce the effect of multipath. In previous chapter, we introduced wavelet analysis as a strong tool for de-noising and multipath estimation. In this chapter, we will discuss other elements which we need to complete our process.

\subsection{Proposed Algorithm}

\subsubsection{Introduction}

In wavelet analysis, there are two major challenges which could affect the analysis. First, it is the level of filtering, and the last is the value of the threshold which was discussed in Chapter 3. Basically, by looking at each level, the amount of low and high frequencies are emerged. In each level we decide how much of a frequency is needed and what should be disregarded. In other words, we decide how much of a low frequency or high frequency has to be filtered. The number of levels is highly depended on the desired application. Since multipath behavior is considered to be randomly distributed, we look for a criteria which could express the randomness. In GPS analysis, Entropy could be considered as a criteria to adaptively select a level for filtering the data. 


\subsubsection{Entropy}

The concept of entropy in information theory describes how much randomness (or alternatively "uncertainty") there is in a signal or in a random event. An alternative way to look at this is to talk about how much information is carried by the signal. As a system gets more random, its entropy goes up. Entropy of a system increases as the system becomes more disordered.

The base of the logarithm determines the units. When we use the base 2 the units are in bits (base 10 gives digits and the base of the natural logarithms $\boldsymbol{e}$ gives nits). Information is always a measure of the decrease of uncertainty at a receiver. Shannon defines entropy in terms of a discrete random event $\boldsymbol{X}$, with possible states (or outcomes) $x_{1} \ldots x_{n}$ as:

$$
E(X)=\sum_{i=1}^{n} p\left(x_{i}\right) \log _{2}\left(\frac{1}{p\left(x_{i}\right)}\right)=-\sum_{i=1}^{n} p\left(x_{i}\right) \log _{2} p\left(x_{i}\right)
$$

where $p\left(x_{i}\right)$ is the probability of the $i^{\text {th }}$ outcome of $X$.

In GPS concept, the randomness or entropy of a signal could be related to the signal distortion. In this chapter, the relationship between the distortion or multipath and entropy is investigated to show a multipath classification. This classification will be used to detect the amount of multipath carried out by a signal and consequently this value will be used to set a number for the levels in wavelet analysis (more details are discussed in Chapter 5). It could be shown that the higher is the entropy value of a signal, the higher is the offert of multipath on the signal. Fig 4.1 shows value of the entropies for a data set. The data set used to plot the Fig. 4.1 has seven satellites available for processing. As shown in Fig. 4.1, each satellite exhibits a different cntropy per satellite, meaning that the condition or channels used to received the signals were different at the given time. Since the atmospheric and hardware errors could be considered constant for the 15 minutes of data collection time, the difference is considered to be caused by different amount of multipaths affecting each signals separately. The data collection for the same place was repeated on different days to see how multipath is behaving in terms of entropy value. A data set consists of eight days of data collection is presented in Fig. 4.2. Entropies of each day for each satellites were computed 


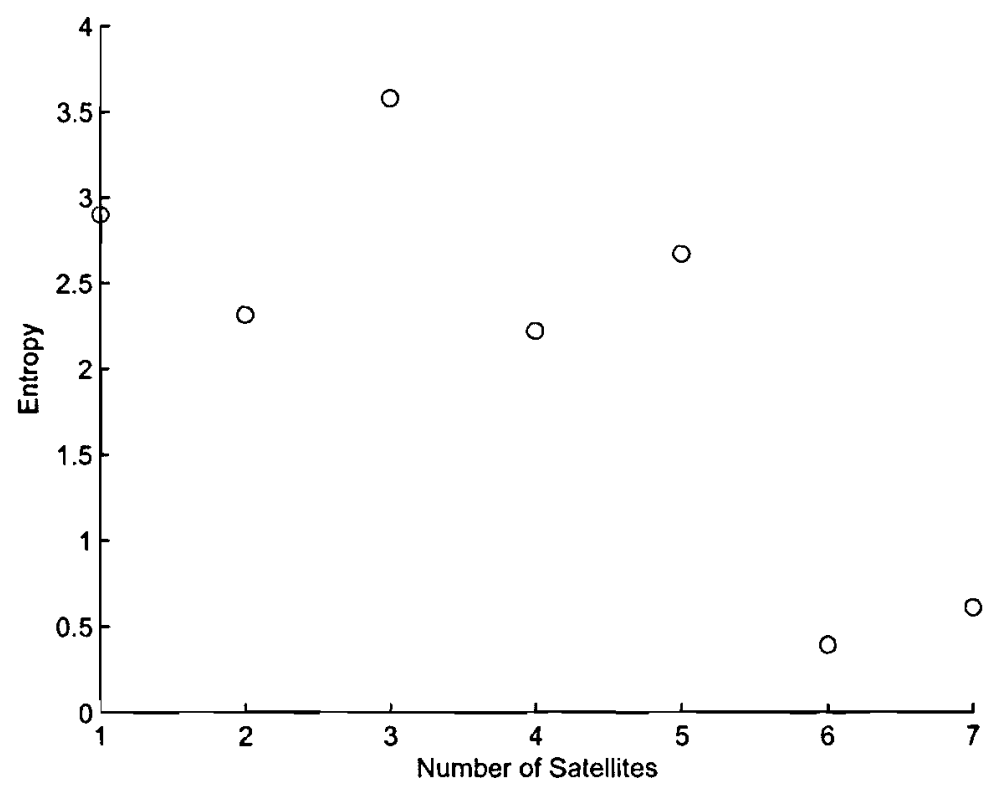

Figure 4.1: Entropies Corresponding to Each of the Satcllites.

and plotted as shown in Fig. 4.2. For example, data set number one (the one marked with plus sign) has eight satellites in view, therefore, there are eight entropies calculated for each satellite. If we follow the markers for each satellite, it could be concluded that the amount of multipath affecting each signal is different in each day. For instance, consider satellite number two. On the first day of measurement, the entropy was calculated to be 3.7 , while on the day of eight, it was computed to be 2.5. In another words, on the day of eight, we had a better condition (less multipath) while collecting the data.

All these entropies, shown in Fig. 4.2, have to be averaged in order to have a clear picture. The simplest method is to average the entropies of each satellite as shown in Fig. 4.3. Therefore, for each satellite there is one entropy. The same procedure was carried out on three different locations as well. Fig. 4.4 shows the averaged entropies for each location. The averaged values were normalized to show the comparison. In Fig. 4.4, the location with averaged entropy of one (red dot) exhibits the highest multipath environment.

Although the computed entropies will be used to classify them, there is another element 


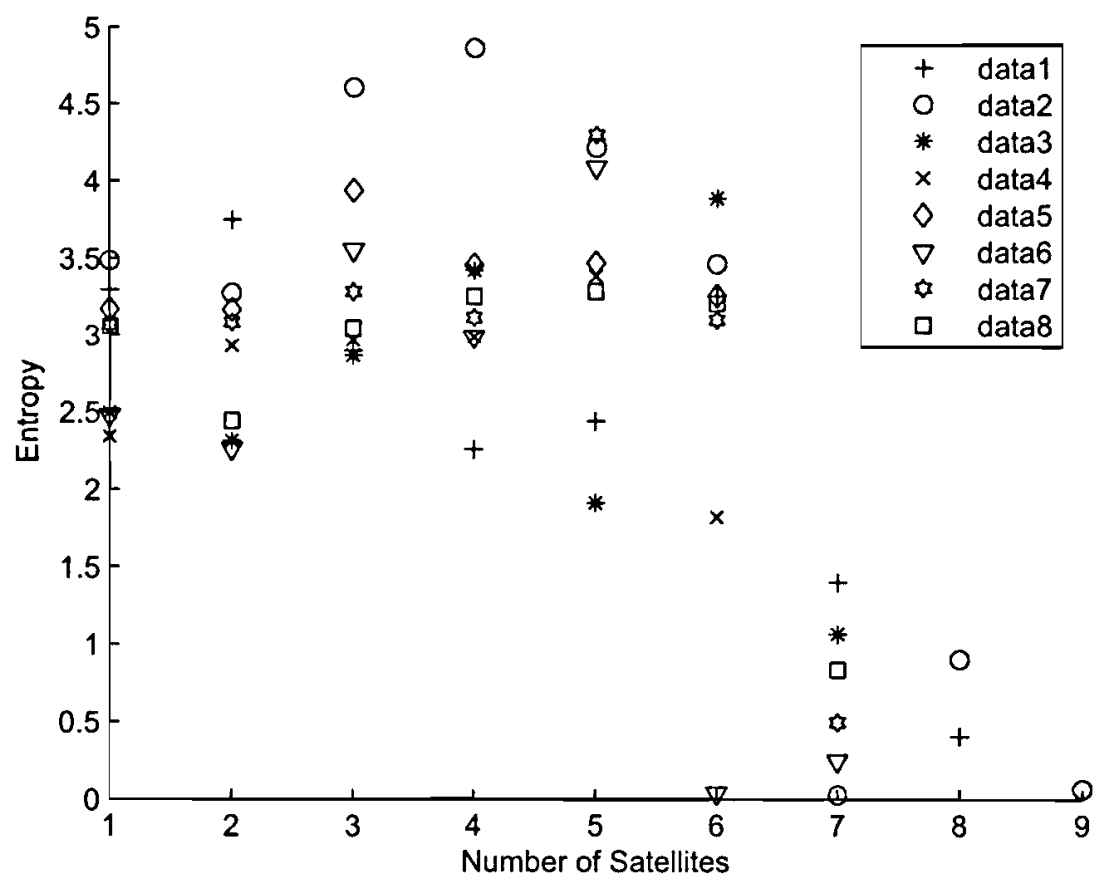

Figure 4.2: Entropies Corresponding to the Whole Data Set.

that has to be looked at before simply taking the average of the entropies. This element is the number of satellites available to be processed by the receiver. Although we may have a high multipath situation, there could be more that 6 satellites in view. The more SV's are available to the receiver, the better the precision of the computed positions will show. Therefore, both the entropy and the number of satellites must be taken into account while classifying them. To classify a location based on the effect of multipath, the norm of the entropies are considered for further processing.

\subsubsection{Norm of the Entropies}

Each data set used in this thesis is in a matrix form of $M \times N$, where $\mathrm{M}$ rows represent the real value data, and the $\mathrm{N}$ columns indicate the number of satellite available during the data collection. Therefore, for each data set there are $\mathrm{N}$ entropies corresponding to each 


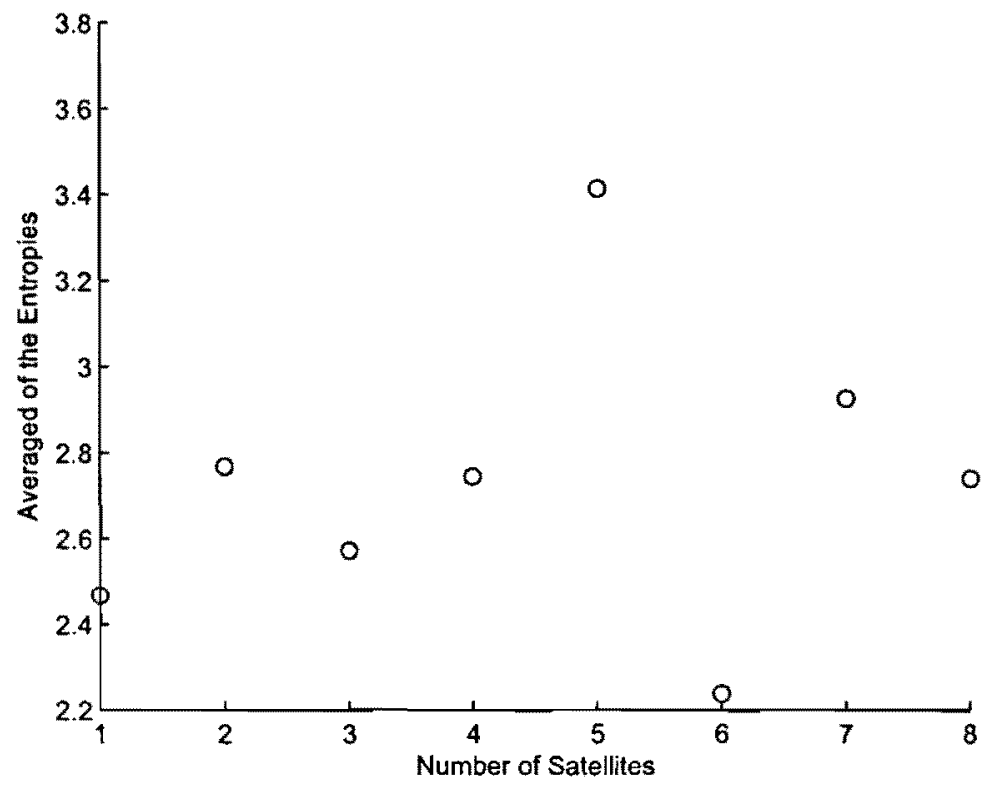

Figure 4.3: Average of Entropies Corresponding to Each Satellites

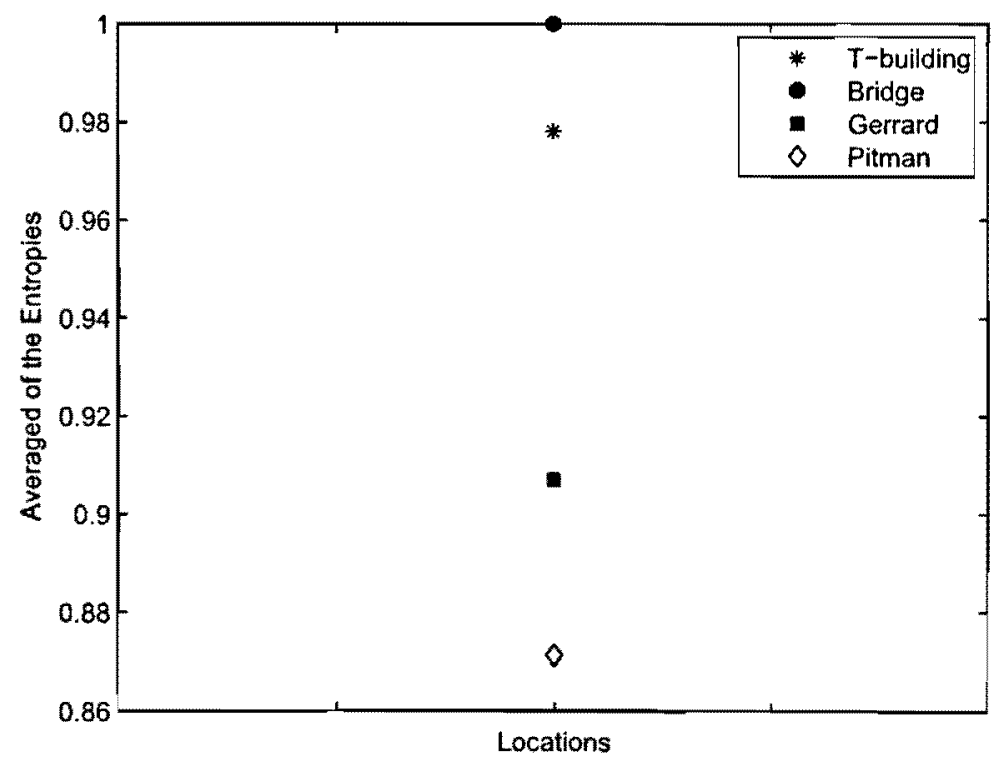

Figure 4.4: Average of Entropies Corresponding to Each Locations 
satellite. The higher is the value of $\mathrm{N}$, the better condition we have in terms of position calculation. Consequently, in addition to entropy, the number of satellites play an important rule in deciding how multipath is effecting the data set. To contribute both the entropy and the number of SV's in an adaptive level selection, the p-norm (Eq. (4.2)) of each data set is calculated.

$$
E_{p}=\left(\sum_{i=1}^{N}\left|E_{i}\right|^{p}\right)^{\frac{1}{p}},
$$

where $\boldsymbol{E}_{\boldsymbol{i}}$ is the entropy of each Sv's, and $\boldsymbol{p}$ is the total number of satellites used for that data set. For example, $900 \times 7$ data set has seven entropies and one $\boldsymbol{E}_{\boldsymbol{p}}$. Table 4.1 shows the computed entropies using Eq. (4.1) for the mentioned example.

\begin{tabular}{cccccc}
\hline SV & 1 & 2 & 3 & 4 & 5 \\
\hline E & 2.85 & 2.88 & 3.52 & 5.31 & 5.71 \\
\hline
\end{tabular}

Table 4.1: Entropies of the Examplc.

$$
E_{p}=\sqrt[5]{2.85^{5}+2.88^{5}+3.52^{5}+5.31^{5}+5.71^{5}}=6.46
$$

Fig. 4.5 shows the norm of the entropies for the same location as Fig. 4.2, computed for the whole data set. The norm of the entropies for four locations were computed. Since for each location we have a few measurements from different days, the mean value of the $\boldsymbol{E}_{\boldsymbol{p}}$ 's were taken and then normalized as shown in Fig. 4.6. In Fig. 4.6, each dot represents a location in down-town Toronto. The red dot illustrates the location with the highest multipath. The adaptive level selection is then based on this classification. For high value of multipath the level is chosen to be 10 , while for the low multipath area the level is selected to be 2 . Before working with real data sets, which is the subject of the next chapter, a synthetic data set was generated, using Matlab, to test the proposed algorithm.

\subsection{Generating Synthetic Signal}

A simulated direct-signal (line-of-sight) could be defined as:

$$
y=\cos \left(\omega_{c} t\right)
$$




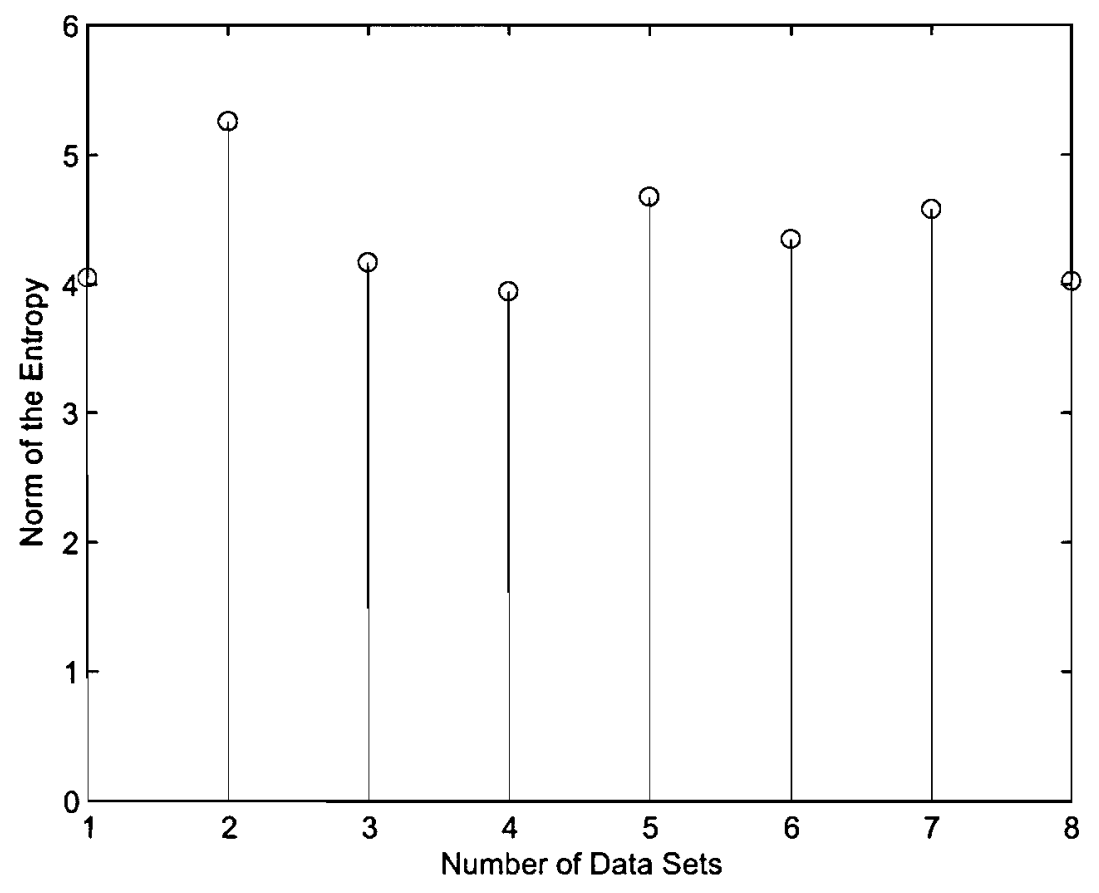

Figure 4.5: Norm of the Entropics.

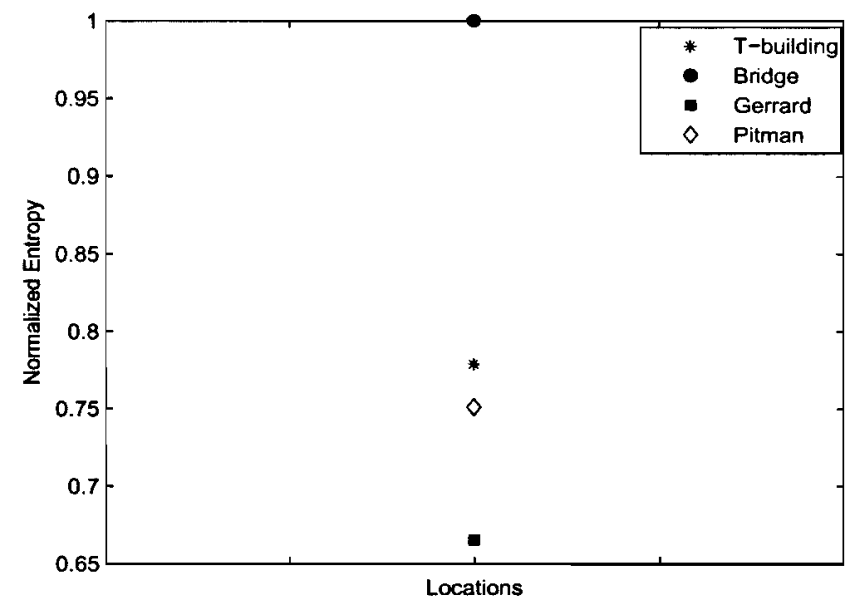

Figure 4.6: Normalized Entropy of the Locations.

where $\omega_{c}$ is the carrier frequency considered to be $1575.42 \mathrm{MHz}$ (same as the L1-band frequency). A multipath signal could be generated by adding random amplitudes and phases 
to the direct-signal, as the line-of-sight signal is passing through a Rayleigh/Rician channel. Fig4.7 shows the generated multipath signals where the more oscillatory waveforms are the

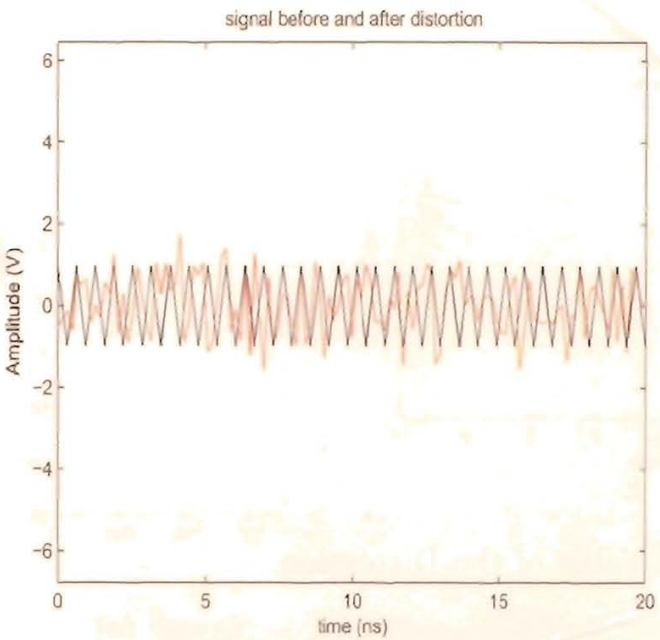

(a) One Path Added to the Direct-Signal.

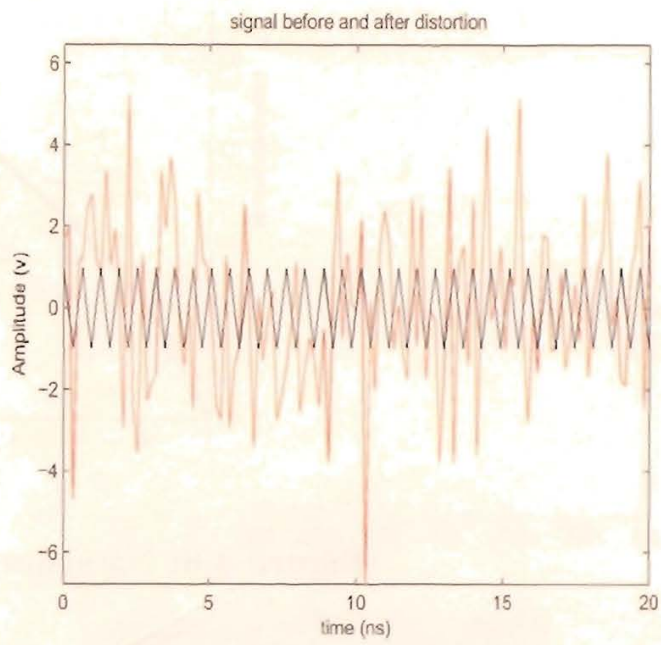

(b) Ten Paths Added to the Direct-Signal.

Figure 4.7: Synthetic Multipath Signal.

distorted signals. Since the amount of distortion added to the line-of-sight signal is known. the proposed algorithm could be tested with the distorted signals. The goal here is to estimate the amount of distortion introduced in the direct-signal. Since the line-of-sight signal is a simple cos function, the amount of distortion is computed by subtracting the original signal from the distorted one. Then the RMS value of the difference is calculated based on Eq. (4.4),

$$
R M S=\sqrt{\frac{1}{N} \sum_{i=1}^{N}\left(S_{\text {distorted }_{i}}-S_{\text {rawi }_{i}}\right)^{2}},
$$

where $\mathrm{N}$ is the signal length and $\boldsymbol{S}_{\text {raw }}$ is the line-of-sight data. The amount of multipath is then estimated by passing the distorted signal, $S_{\text {distorted }}$, through the wavelet filter algorithm. The estimated RMS values are then compared with the true RMS values, as shown in Fig. 4.8. In Fig. 4.8, the red (lighter) color represents the true RMS values while the darker curve illustrates the estimated one. Fig. 4.8 is an indication that the proposed algorithm is operating properly since the true values and the estimated values are almost overlapped. 


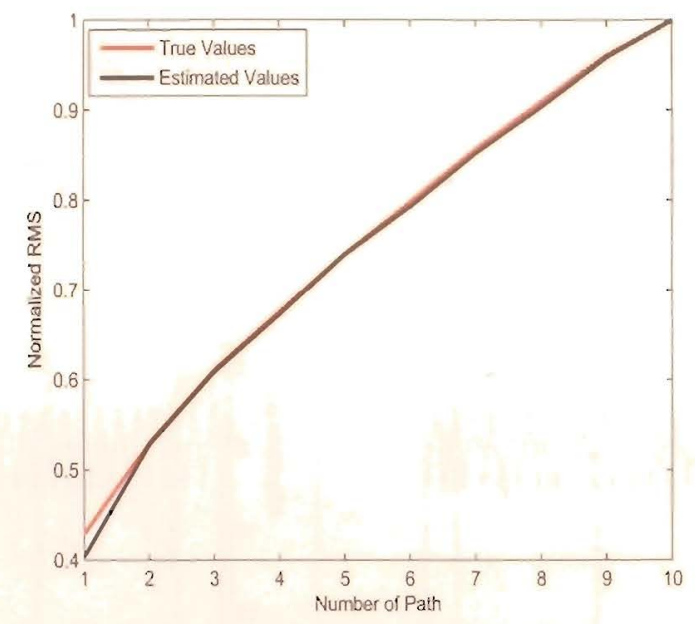

Figure 4.8: Normalized RMS Value of the Distortion.

\subsection{Applying the Proposed Algorithm on the Real GPS Data}

To observe the effect of the proposed threshold value along with the wavelet level selection, residuals of the data set were passed through the wavelet analysis tool. Four samples of the filtered data are shown in Fig. 4.9 and Fig. 4.10. Fig. 4.9(a) and 4.9(b) are representing two samples of the bad data since they carry spikes such as signals with high amplitudes. These type of signals degrade the positioning and must be ignored during the position calculations. As shown in Fig. 4.9, the filtered data is computed to be zero, meaning that the satellite will not be used in positioning. Although there are moments which the values of the data is reasonable to be used, for example in Fig. 4.9(b) around 200 (s), it is safer not to use the satellite if more than four SV's are available.

On the other hand, when the data is good the analysis approximates the distortion as shown in Figs.4.10(a) and 4.10(b). In these figures, the red (lighter) color represents the data before filtering and the darker one illustrates the data after being filtered. 


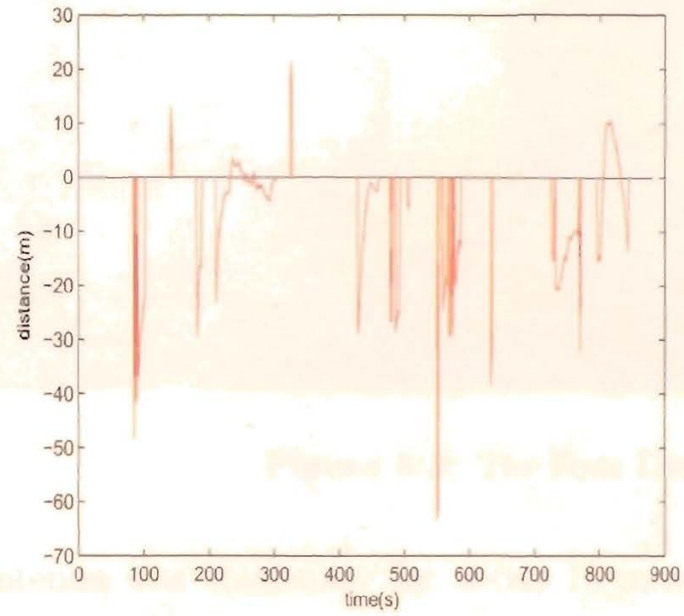

(a) Sample of Bad Data, PRN6.

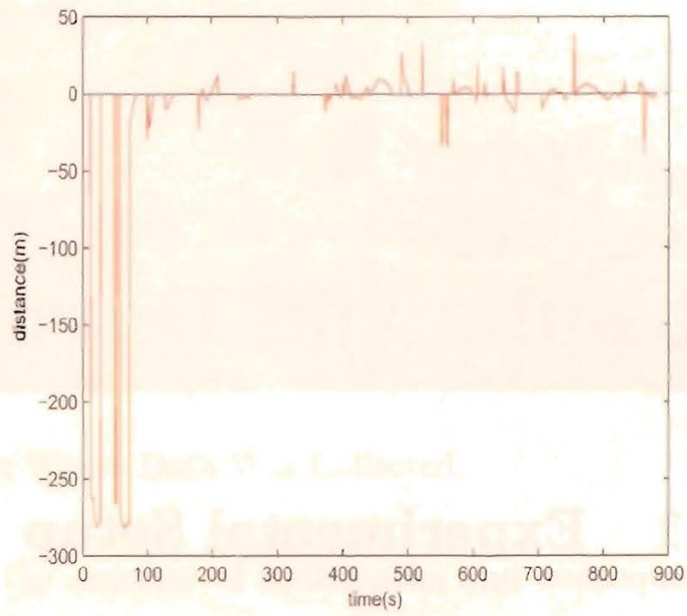

(b) Sample of Bad Data,PRN9.

Figure 4.9: Wavelet Analysis of a GPS Data.

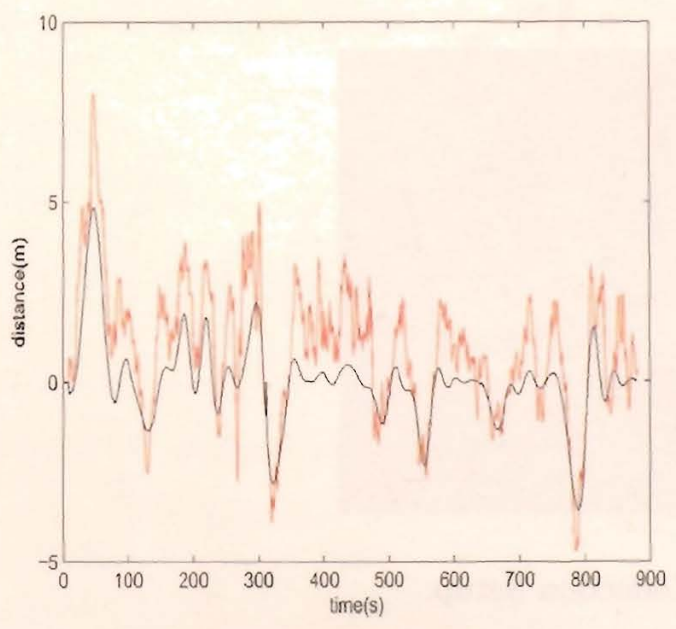

(a) Sample of Good Data, PRN10.

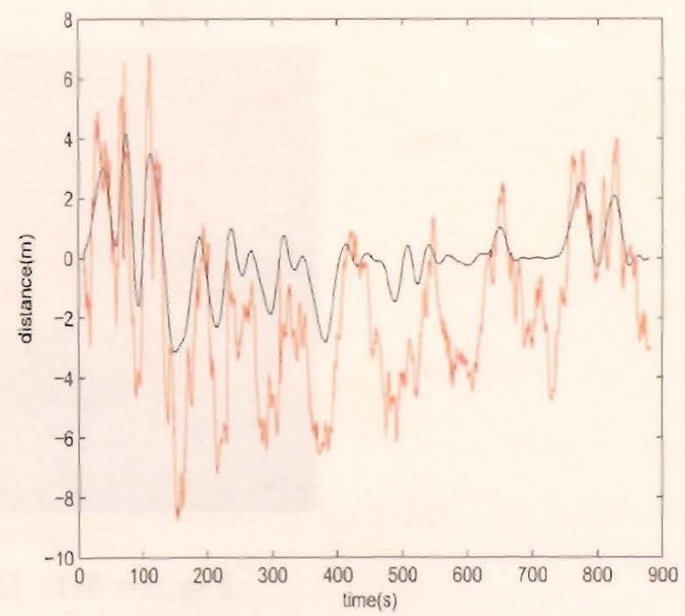

(b) Sample of Good Data,PRN5.

Figure 4.10: Wavelet Analysis of a GPS Data. 


\section{Chapter 5}

\section{Results and Discussions}

\subsection{Experimental Setup}

To evaluate the effect of the new threshold value and the wavelet level selection on the calculated positions, 29 data sets were collected in downtown Toronto. In this experiment data collection equipment consisted of: u-blox TM-LP15 evaluation kit with u-center ANTARIS software and a laptop. An active antenna was mounted on a portable antenna that was mount one meter above the ground. We did not use an external ground plane [25]. Fig. 5.1 shows the receiver setup used to collect data. The data was collected in four different

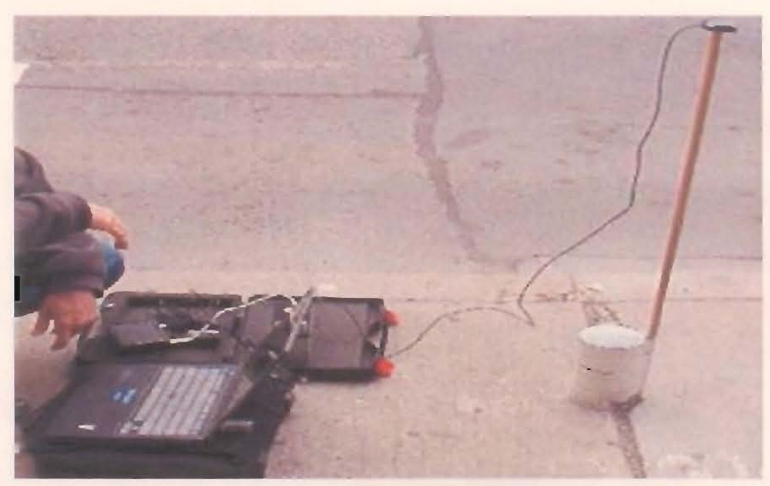

Figure 5.1: Data Collection Setup.

regions, each exhibiting different amount of multipaths in terms of low to high degree of multipath. Fig. 5.2 shows the four chosen locations. The circle in each photo illustrates the location of the receiver. Although in all four locations the positions of the receiver or the 


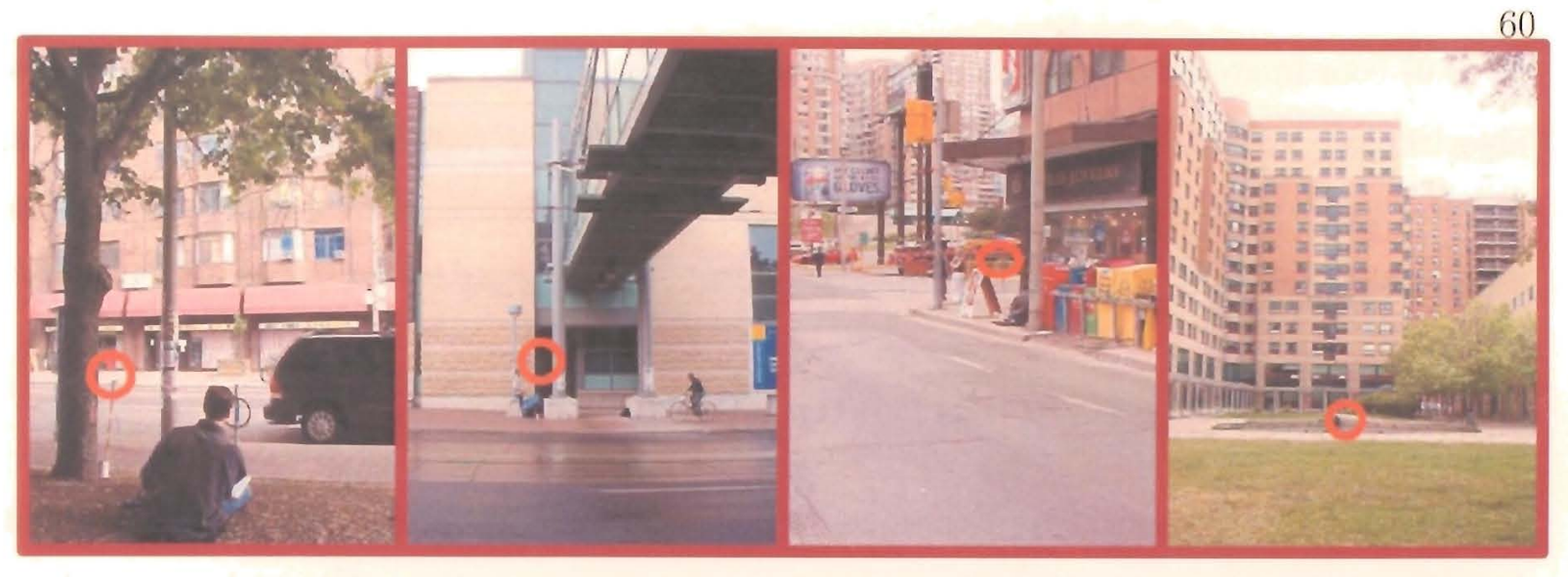

Figure 5.2: The Four Locations Where Data Was Collected.

antenna was stationary for about 15 minutes, the amount of multipaths and consequently the coordinates of the user was changing with time as if the user was in motion. Fig. 5.3

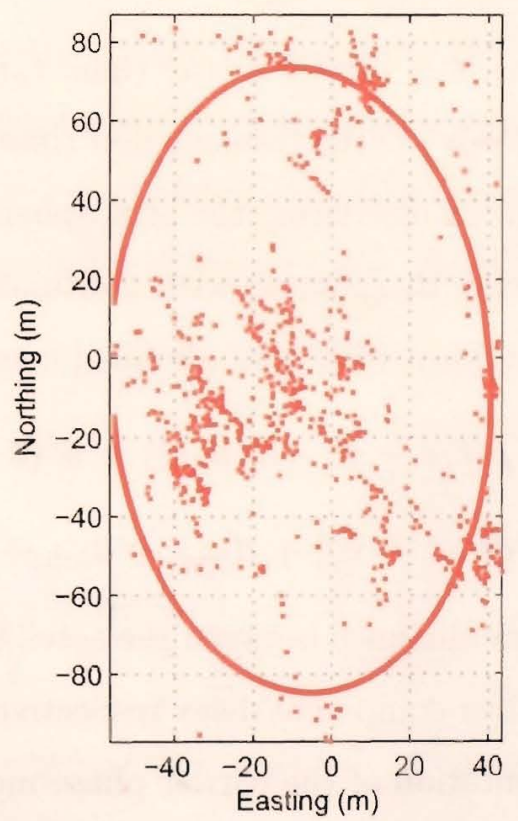

Figure 5.3: Easting and North Errors of the Second Location, from Right-side of Fig. 5.2.

shows the mentioned fact that even though the user is in stationary position, the computed coordinates are not constant. In Fig. 5.3 each dot represents a position which is normalized 
to $(0,0)$ coordinate, and the confidence ellipse is plotted based on $3 \sigma$. In this thesis a new algorithm as discussed in Chapters 3 and 4 are designed to reduce the scatter points, in another words, the algorithm minimize the effect of the multipath errors and reduces the standard deviation of the computed positions.

\subsection{Residual (Signal) Modeling}

In order for a GPS receiver to determine its position, it has to receive time signals from at least four different satellites [2]. Then pseudoranges, from code $\left(\boldsymbol{y}_{\text {code }}(t)\right)$ and carrier phase $\left(\boldsymbol{y}_{\text {phase }}(t)\right)$, of the user from the satellites can be determined with the help of signal transmit times between the satellites and the receiver [2]. These pseudoranges are computed from both pseudo random code $(P R N)$ and carrier phase information as:

$$
y_{\text {code }}(t)=c\left[t_{r}(t)-t^{s}(t-\tau)\right]+\epsilon_{p},
$$

where $c$ is the speed of the light, $t$ is the GPS true time, $t_{r}(t)$ is the reception time, $\tau$ is the total signal travel time, $\boldsymbol{t}^{s}(\boldsymbol{t}-\boldsymbol{\tau})$ is the transmission time from a satellite, and $\boldsymbol{\epsilon}_{\boldsymbol{p}}$ is the error added to the measurements. Considering the atmospheric effect including ionospheric

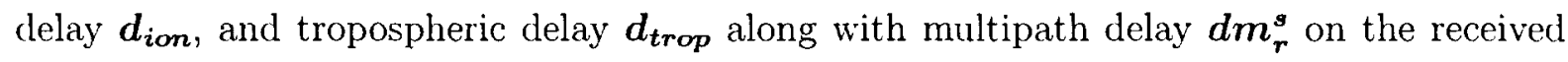
signals, Eq. (5.1) can be broken down with more detailed components [2].

$$
\begin{gathered}
y_{\text {code }}(t)=\rho(t, t-\tau)+c\left[d_{r}(t)+d^{s}(t-\tau)\right] \\
+c\left[d t_{r}(t)-d t^{s}(t-\tau)\right]+d_{\text {trop }}+d_{\text {ion }}+d m_{r}^{s}+\epsilon_{p} .
\end{gathered}
$$

Here, $\rho(t, t-\tau)$ is the geometric distance between the satellite and the observing point, $d^{s}$ and $d_{r}$ are the satellite and receiver equipment delay respectively, and $d t_{r}(t)$ is the reception time. Eq. (5.3) shows a representation of the carrier phase measurements [2],

$$
\begin{array}{r}
y_{\text {phase }}(t)=\rho(t, t-\tau)+c\left[\delta_{r}(t)+\delta^{s}(t-\tau)\right]+d_{\text {trop }} \\
+c\left[d t_{r}(t)-d t^{s}(t-\tau)\right]+d_{i o n}+\lambda\left[\phi_{r}\left(t_{0}\right)-\phi^{s}\left(t_{0}\right)\right]+\lambda N+\delta m_{r}^{s}+\epsilon_{\phi}
\end{array}
$$

where $\delta_{r}(t), \delta^{s}(t), \phi_{r}\left(t_{0}\right)$, and $\phi^{s}\left(t_{0}\right)$ are the carrier phase equipment delays and the initial phases of the receiver and satellite carrier signal respectively, $\boldsymbol{\lambda}$ is the wavelength and $N$ is 
an integer representing the carrier phase ambiguity. The resultant time error caused by the error sources creates inaccuracies in the measurement. Among the errors multipath creates an incremental time delay, causing the respective pseudoranges $\left(\boldsymbol{y}_{\text {code }}(\boldsymbol{t})\right)$ to be incorrect [6]. The input data to be analyzed is a set of residuals (i.e. code minus carrier) given by Eq. (5.4).

$$
\begin{aligned}
y(t) & =y_{\text {code }}(t)-y_{\text {phase }}(t) \\
\Rightarrow y(t) & =2 d_{\text {ion }}+d m_{r}^{s}-\lambda N
\end{aligned}
$$

When such a difference is performed, all common errors such as satellite clock error, tropospheric error, and receiver clock error are eliminated. What remains is predominantly twice the ionospheric error, the pseudorange multipath error, and the carrier phase ambiguity. The carrier phase multipath $\left(\delta \boldsymbol{m}_{\boldsymbol{r}}^{\boldsymbol{s}}\right)$ is ignored since its value is very small compared to pseudorange multipath. The ambiguity term could be seen as a bias value which is removed by taking the mean value of the observations and subtracting the mean from the data. Therefore, the remaining values represent the ionosphere plus the multipath errors. To approximate the multipath error, wavelet analysis tool is applied to the remaining sum. Fig. 5.4 illustrates two typical GPS residuals. Fig. 5.4(a) shows 900 seconds of a normal

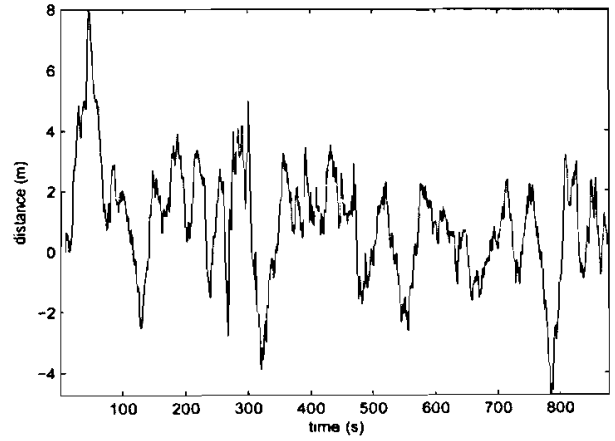

(a) Sample of Good Data.

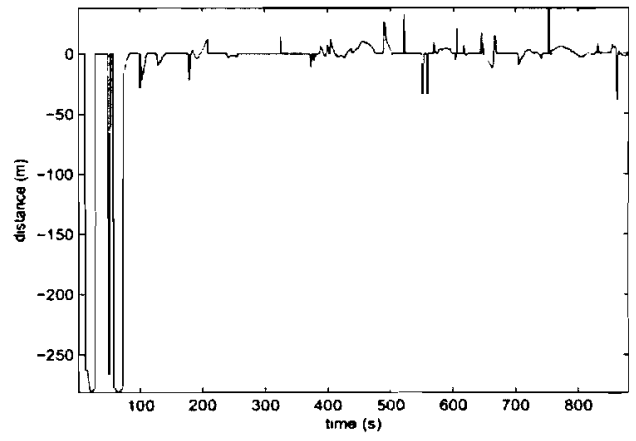

(b) Sample of Bad Data.

Figure 5.4: Typical Code-Carricr Data.

data, while Fig. 5.4(b) contains high values of multipath. The high amplitudes (spikes or transients like) indicate that data carries strong multipath signals. An approach to mitigate 
this strong multipath is by using a time varying approach such as wavelet analysis. This is discussed in the next section.

\subsection{Performance Analysis}

To evaluate the performance of the proposed algorithm three statistical elements namely standard deviation, kurtosis, and skewness of both raw and filtered positions are computed, and then they are compared to show the degree of improvements or degradations. In that regard, the two major characteristics of a multi-resolutional analysis, thresholding and number of levels, are separately discussed.

\subsubsection{Wavelet Analysis on Residuals, Thresholding}

In order to be consistent with the signal modeling approach proposed by Donoho [24], Eq. (5.4) can be simplified to a standard non-parametric regression problem as:

$$
y\left(t_{i}\right)=x\left(t_{i}\right)+n_{i}, \quad i=1,2, \ldots, k,
$$

where $y\left(t_{i}\right)$ represents the code minus carrier signal (from the measurement), $x\left(t_{i}\right)$ is the signal we wish to approximate, and $n_{i}$ is considered to be noise added to the system. As shown in Fig. 3.7, the raw data, $y\left(t_{i}\right)$, is decomposed by wavelet filtering to details and approximations. Thresholding is then applied to the coefficients to keep the approximations and disregard the insignificants. Doncho [24] showed that such wavelet estimators with a properly chosen threshold value have various important optimality properties. The choice of the thresholding value, therefore, becomes a crucial step in the estimation procedure. The observables pseudorange and carrier phase information were obtained from the receiver, and then residuals were computed as expressed in Eq. (5.4). These residuals data were filtered with both Donoho's estimator Eq. (3.22), and the proposed threshold value estimation Eq. (3.35) to mitigate the effect of multipaths on the pscudoranges and consequently have a better precisions on the computed positions. Figs.5.5 and 5.6 show two samples of the computed positions. The lighter (red) dots represent the positions from the raw data 
(data without filtering), and the darker dots show the final positions after data has been filtered. The darker dots in Fig. 5.5(a) show the filtered positions as Eq. (3.22) was used to threshold the wavelet coefficients. Obviously in Fig. 5.5(a), the final positions have a much greater standard deviation compared to the raw positions (the lighter dots). In order to

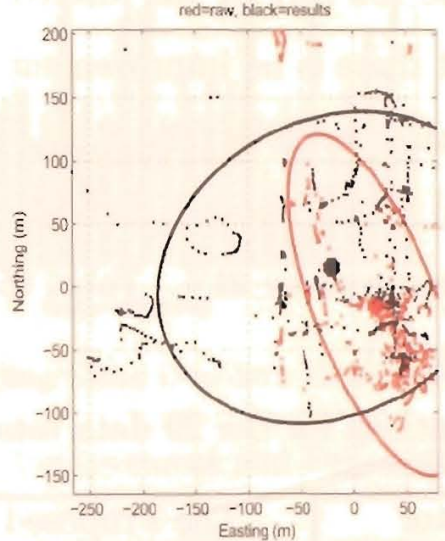

(a) Thresholding by Eq. 3.22.

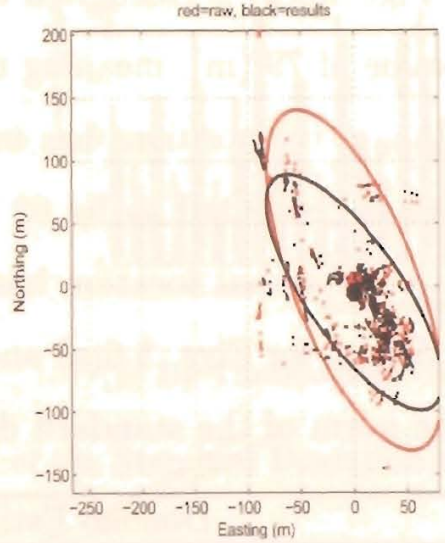

(b) Thresholding by Eq. 3.35 .

Figure 5.5: Comparison of the Positions Precision Resulted from Eqs. 3.22 and 3.35.

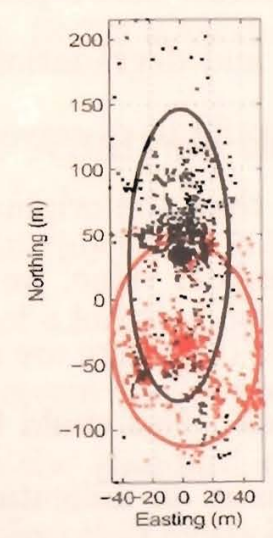

(a) Thresholding by Eq. 3.22.

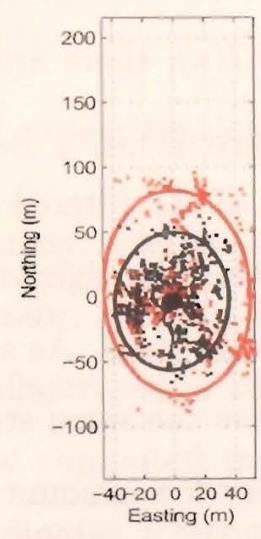

(b) Thresholding by Eq. 3.35.

Figure 5.6: Comparison of the Positions Precision Resulted from Eqs. 3.22 and 3.35, Another Location.

quantify the results, the norm of the standard deviation of the longitudes and latitudes of 
the positions are taken as expressed in Eq. (5.6).

$$
S_{n o r m}=\sqrt{\text { std(longitudes })^{2}+\operatorname{std}(\text { latitudes })^{2}}
$$

The lower the value of $S_{\text {norm }}$, the lower the data is scattered (lower standard deviation). The $\boldsymbol{S}_{\text {norm }}$ of the Fig. 5.5(a) is calculated to be 102 (i1) for the filtered data, whereas the raw data has the value of $79(\mathrm{~m})$, meaning that there is no improvement in this case. This shows that the $\boldsymbol{S}_{\text {norm }}$ of the positions has increased by $29 \%$. The $\boldsymbol{S}_{\text {norm }}$ of the Fig. $5.5(\mathrm{~b})$, for the filtered data is computed to be $62(\mathrm{~m})$ which implied that with our filtering the $S_{\text {norm }}$ of the positions for that location has improved by $21 \%$. Tabel $(5.1)$ represents the comparison of the $S_{\text {norm }}$ for Figs. 5.6(a) and 5.6(b).

Fig. 5.7 shows the norm of the standard deviations for the 29 data sets. In Fig. 5.7, the

\begin{tabular}{ccc|cc}
\hline & \multicolumn{2}{c|}{ Donoho Estimator } & \multicolumn{2}{c}{ The Proposed } \\
\hline & Raw Data & Filtered Data & Raw & Filtered Data \\
$\boldsymbol{S}_{\text {norm }}(\mathrm{m})$ & 46 & 58 & 46 & 32 \\
\hline
\end{tabular}

Table 5.1: Comparison of the $S_{\text {norm }}$ for the Location Shown in Fig. 5.6.

lighter color (grey) represents the raw data, while the darker bars represent the filtered data. As illustrated in Fig. 5.7(a), there are improvement and degradation when the threshold value is set to the Donoho's estimator, Eq. (3.22). It could be observed that there are cases that standard deviation of the filtered data is higher than the original data (the data not being filtered). While using the eigenvalue as the threshold value, we observe that there is no degradation over the raw data. As shown in Fig. 5.7(b), there are a few cases that both raw and filtered data have the same standard deviation. This could be from the fact that these data sets carry very low amount of errors. This is an indication that the proposed algorithm is operating properly. If the presence of multipath is negligible, meaning that the effect of line-of-sight signals are much stronger that the multipaths, the algorithm does not affect the data, while by applying the Donoho's estimator as the threshold value, the results are changing regardless of the density of the errors. In another words, when multipaths are present in a data set, the algorithm detects them by properly thresholding the signals, and 


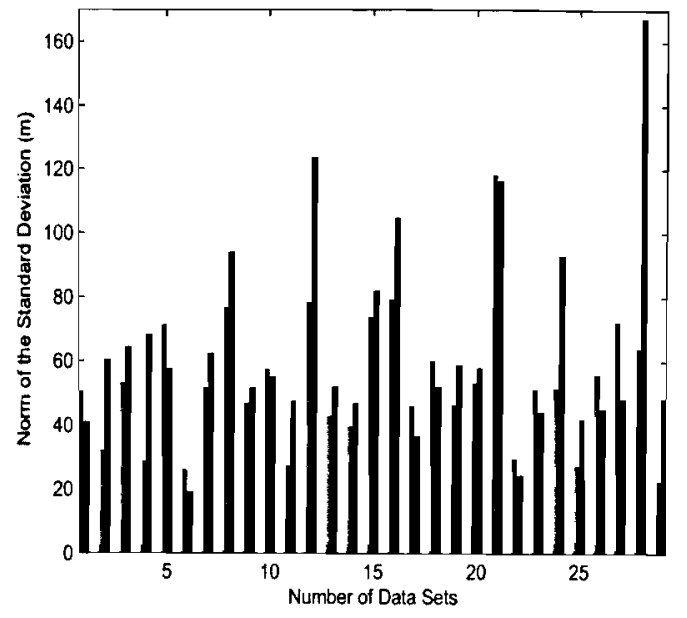

(a) By Thresholding Using Donoho's Estimator.

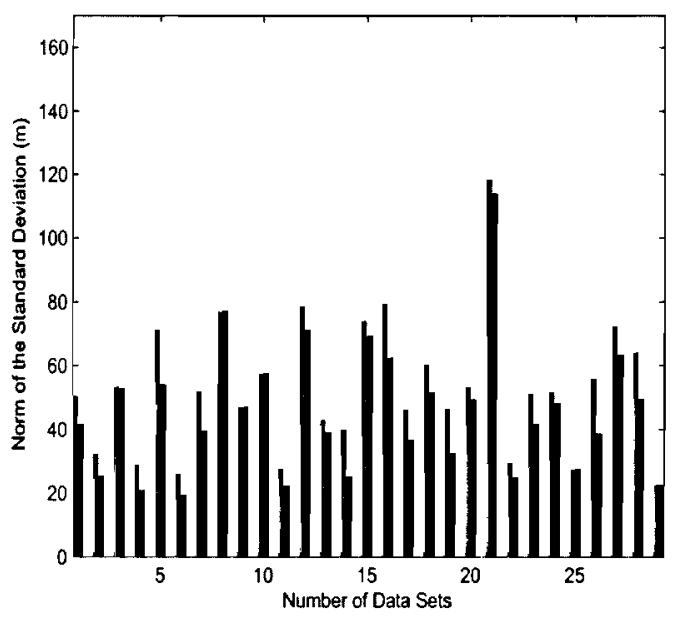

(b) By Thresholding Using Eigenvalue.

Figure 5.7: Improvement and Degradation of the Standard Deviation of 29 Data Scts.

then mitigates them through multi-resolution analysis which adaptively select the number of levels necessary to extract the errors. Furthermore, comparing the Figs5.7(a) and 5.7(b) by each corresponding data sets thresholded by Donoho's estimator and eigenvalue, we observe that in all cases standard deviation resulted from eigenvalue thresholding is less than what is obtained when Eq. (3.22) is used.

\subsubsection{Wavelet Analysis on Computed Positions}

Although the work in this thesis is based on stationary receiver/antenna, as shown in Fig. 5.3 , the coordinates of a location does not stay constant. One way to decrease the deviations was to mitigate the errors on the pseudoranges as illustrated in Fig. 5.7(b).

To further decrease the standard deviation of the computed positions and lowering the virtual motion (velocity) that exists among the coordinates, a second stage multi-resolutional analysis is applied on the calculated positions. 


\section{The Effect of Levels on Standard Deviation}

As the threshold plays an important rule in wavelet filtering, the number of levels determines the resolution of the filtered data, which is another major characteristics of the multiresolution analysis. The high frequencies in a signal could be considered as noise added to the signal. By selecting higher levels, basically we are trying to remove more of the high frequencies in each levels.

The coordinates of a location could be seen as low frequencies (information), and the gaps that exist between the points are considered to be high frequencies. In terms of physical meaning, these gaps resemble the velocity of an object moving randomly without any destination, as shown in Fig. 5.3. Since the objective of positioning is to have a very low standard deviation (less than $2 \mathrm{~m}$ ), wavelet filtering is used on the position points. The same locations

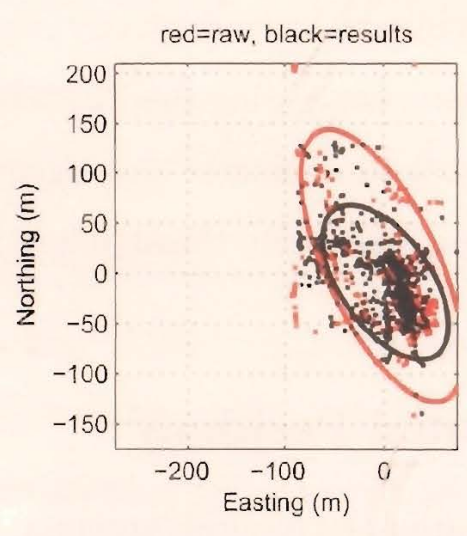

(a) Same Location as Fig. 5.5.

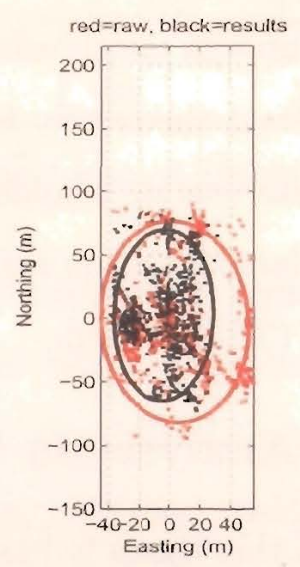

(b) Same Location as Fig. 5.6.

Figure 5.8: Effect of Leveling on the Scatter Points, level $=2$.

as shown in Figs.5.5 and 5.6, are selected to be filtered with the different number of levels. Fig. 5.8 shows the scatter changes. The deviations seen in standard deviations are illustrated in Fig. 5.9(a). To have a quick access to the amount of changes, cumulative distribution function (cdf) of the standard deviations are plotted as shown in Fig. 5.9(b). In Fig. 5.9(b), the dotted line represents the cdf of the raw data, while the solid line shows the cdf of the filtered data. The higher the gap between the dotted line and the solid line, the better are 


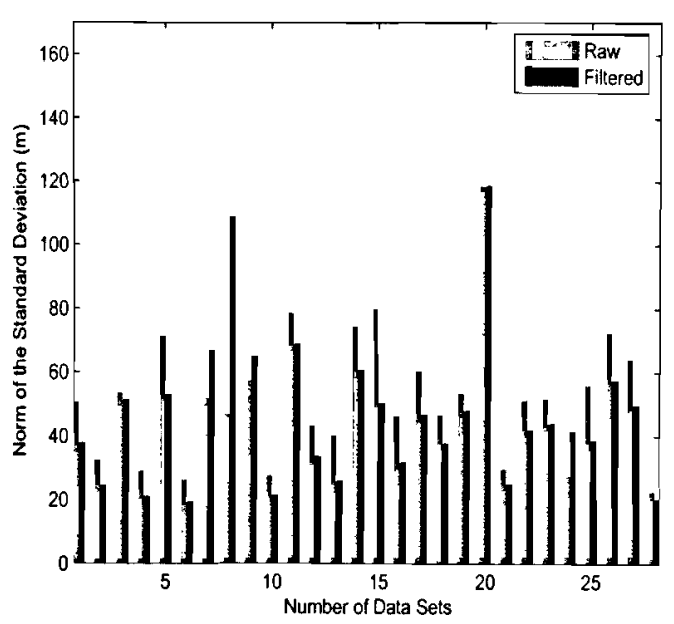

(a) Standard Deviation Improvements/Degradation

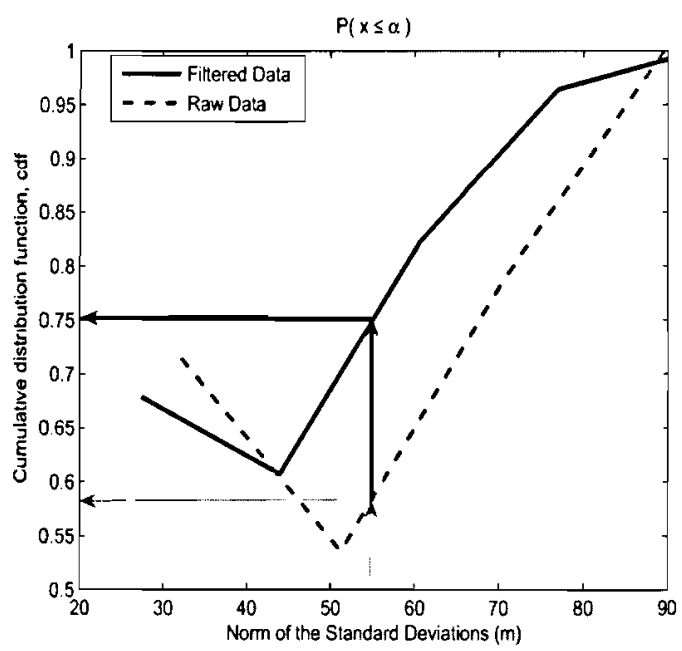

(b) cdf Improvements.

Figure 5.9: Norm and Cumulative Distribution Function (cdf) Improvements.

the results. For example, we are looking for norm of the standard deviation to be $55 \mathrm{~m}$. There are two choices to be considered, as shown in Fig. 5.9(b). Without filtering the data, we have $58 \%$ chance to hit the target $(55 \mathrm{~m})$, while with filtering the data the probability goes higher to $75 \%$.

Since the object of this thesis is to obtain a standard deviation of less than $2 \mathrm{~m}$ for $95 \%$ of the time, the number of levels was increased to 10. Fig. 5.10 shows the same locations as before (Fig. 5.5) to have a visual comparison between the levels and the scattering points. We observe that there is a noteable difference between the scatter points from level 2 to 10. By a simple visual inspection we can observe that the standard deviation has improved significantly to the point that the objective of the proposed research work has been reached. The total improvements are plotted and shown in Fig. 5.11. As mentioned before, in the bar plots, the raw data is presented by a lighter color (grey) while the results are the darker ones. Since the results are very small compared to the raw data, the zoomed version of the results are shown in Fig. 5.12(a). The cdf of the results are shown in Fig. 5.12(b). As shown in Fig. 5.12(b), with a probability of about $96.55 \%$, the norm of the standard deviation will be less than $1.5 \mathrm{~m}$. In another words, there is only one case that shows the norm to be 2.2 

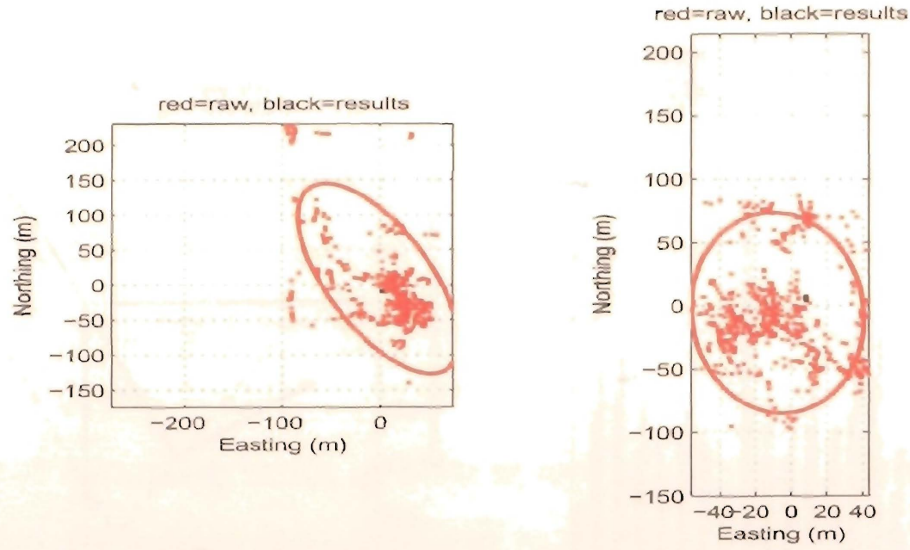

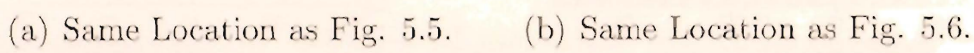

Figure 5.10: Effect of Leveling on the Scatter Points, level=10.

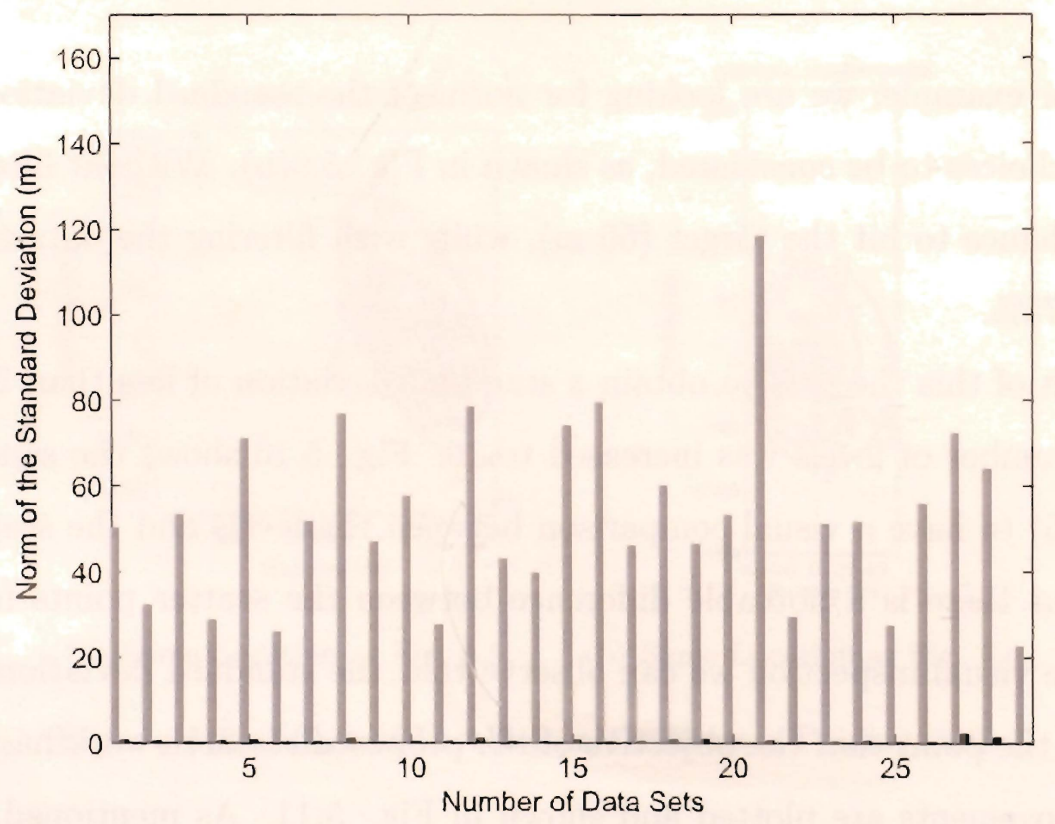

Figure 5.11: Norm of the Standard Deviation Improvements, Level=10.

$\mathrm{m}$, and the rest of the data sets show a norm of less than $1.5 \mathrm{~m}$.

In addition to the standard deviation, two other statistical elements namely Kurtosis and Skewness of the data sets were computed to investigate the distributed scatter points. 


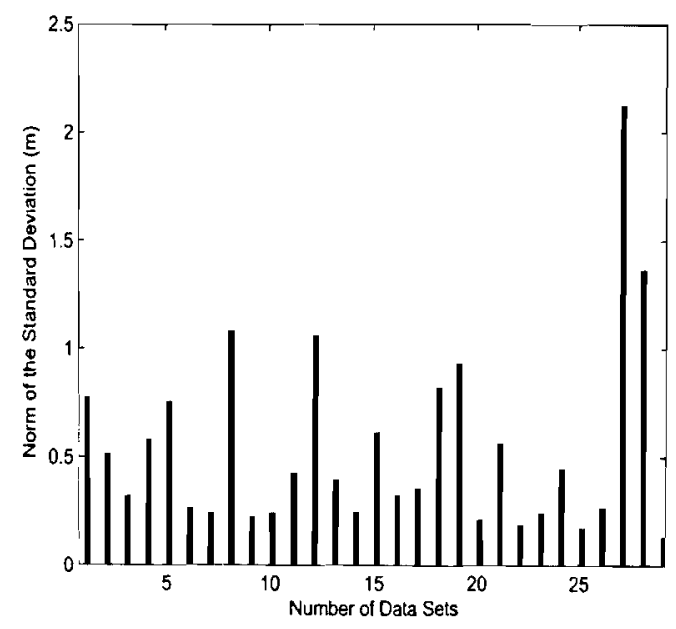

(a) Zoomed Version of the Filtered Data.

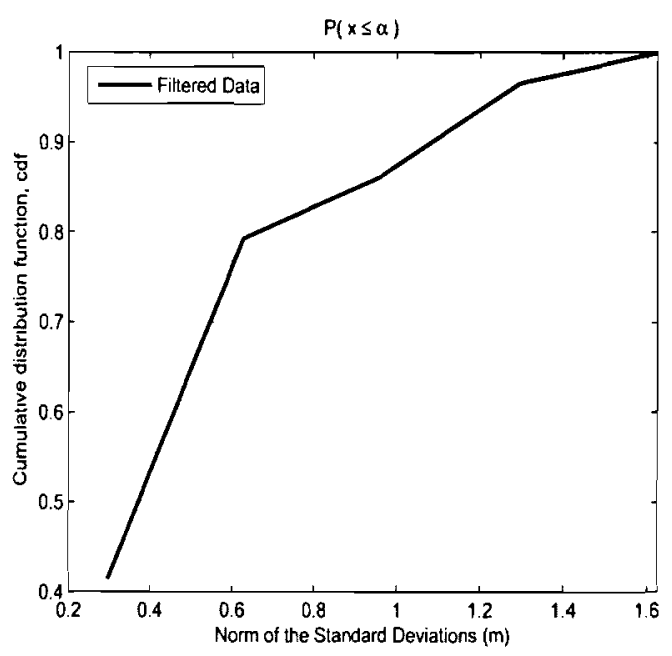

(b) cdf Improvements.

Figure 5.12: Final Norm and Cumulative Distribution Function (cdf) Improvements.

Kurtosis of a clata set is computed to observe how outlier-prone a distribution is. In probability theory and statistics, kurtosis is a measure of the "peakness" of the probability distribution of a real-valued random variable. Higher kurtosis means more of the variance is due to infrequent extreme deviations, as opposed to frequent modestly-sized deviations. For example, the kurtosis of the normal distribution is 3 . Distributions that are more outlierprone than the normal distribution have kurtosis greater than 3; distributions that are less outlier-prone have kurtosis less than 3 [22]. The kurtosis of a distribution is defined as:

$$
k=\frac{E(x-\mu)^{4}}{\sigma^{4}}
$$

where $\boldsymbol{\mu}$ is the mean of $\boldsymbol{x}, \boldsymbol{\sigma}$ is the standard deviation of $\boldsymbol{x}$, and $\boldsymbol{E}($.$) represents the expected$ value of the quantity (.). Some definitions of kurtosis subtract 3 from the computed value, so that the normal distribution has kurtosis of 0 .

As shown in Fig. 5.13, about $95 \%$ of the raw data shows a norm Kurtosis of greater than 3 , while the results are $100 \%$ below 3 . The average value of the Kurtosis is calculated to be $2.8 \mathrm{~m}$. This value indicates that the filtered data distribution could be considered as normal distribution. The last statistical elements to be computed is Skewness of the data. Skewness 


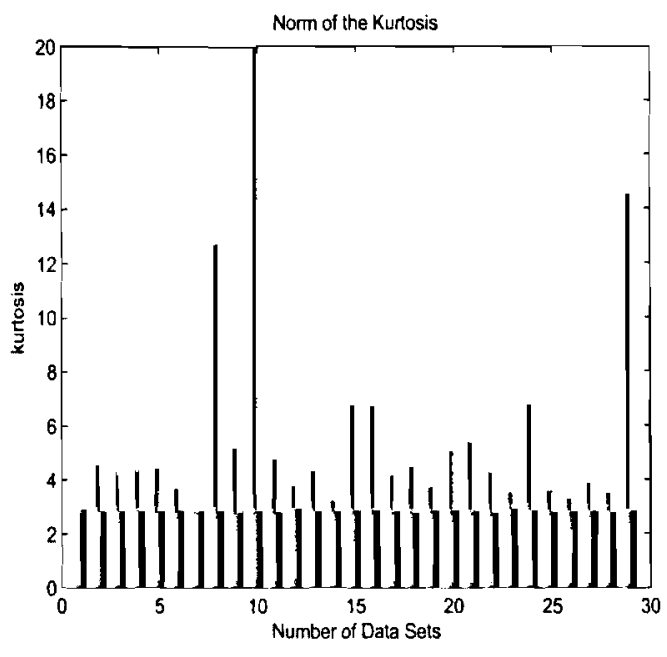

(a) Kurtosis of the Data Sets.

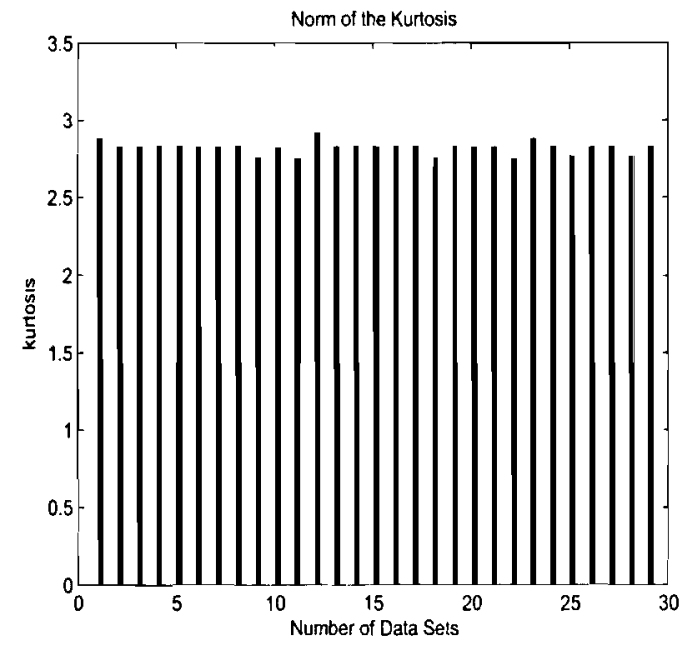

(b) Kurtosis of the Results, Zoomed

Figure 5.13: Kurtosis of the Whole Data Sets.

is a measure of the asymmetry of the data around the sample mean. If skewness is negative, the data are spread out more to the left of the mean than to the right. If skewness is positive, the data are spread out more to the right. The skewness of the normal distribution (or any perfectly symmetric distribution) is zero [22]. The skewness of a distribution is defined as:

$$
y=\frac{E(x-\mu)^{3}}{\sigma^{3}}
$$

As shown in Fig. 5.14, while the norm of Skewness of the raw data (lighter color) is varied from data set to data set, the skewness of the filtered data is almost constant. The variation makes it difficult to calibrate the computed positions in terms of accuracy of the coordinates as in case of raw data. When the data sets are exhibiting a uniform skewness, this feature enables us to calibrate the algorithm in case the computed coordinates show deviation from the true coordinates. At the end, coordinates of two locations in down town Toronto were mapped to compare the raw and filtered positions, as shown in Figs.5.15 and 5.16. In these figures, the blue dots (darker color) are the raw coordinates, and the lighter color dots represent the positions after being filtered. Fig. 5.15(b) shows the zoomed version of the Fig. 5.15(a) to illustrate the tightness of the results compared to the raw data. As noted 


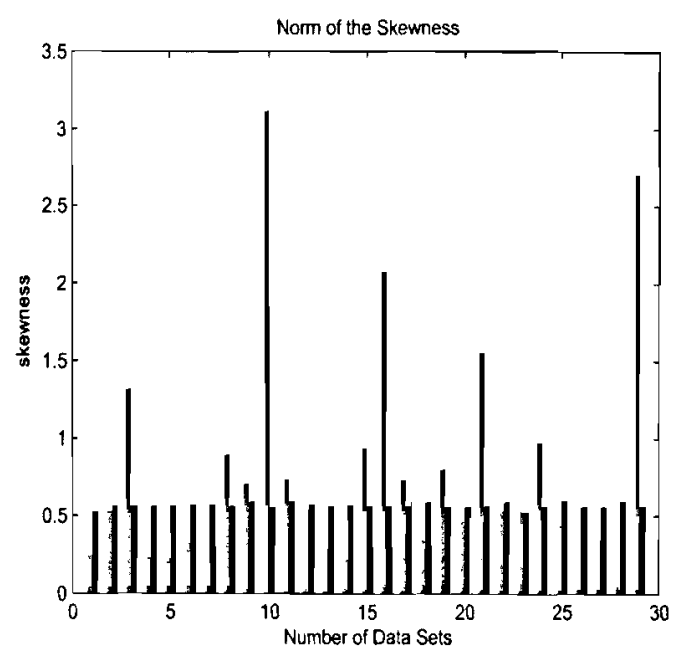

(a) Skewness of the Data Sets.

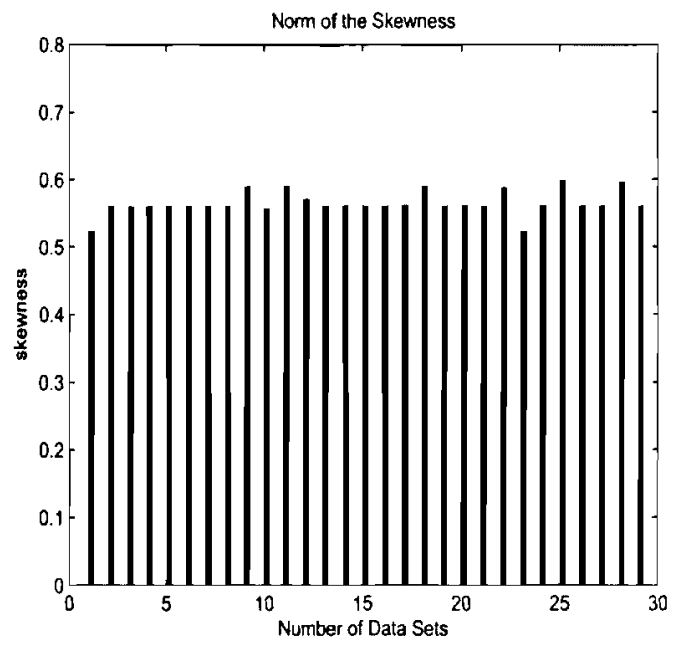

(b) Skewness of the Results, Zoomed

Figure 5.14: Skewness of the Whole Data Sets.

in Fig. 5.15(b), the maximum deviation for this location is about $2 \mathrm{~m}$. Fig. 5.16 illustrates another location, which exhibits a much less standard deviation comparing with the raw data (blue color). In Fig. 5.16(a), the desired position is shown by a dark square. Fig.

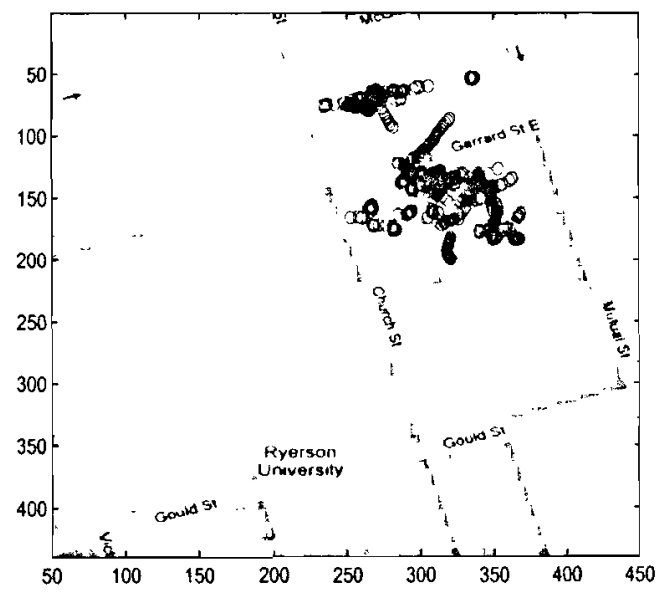

(a) Final Positions, Raw and Filtered.

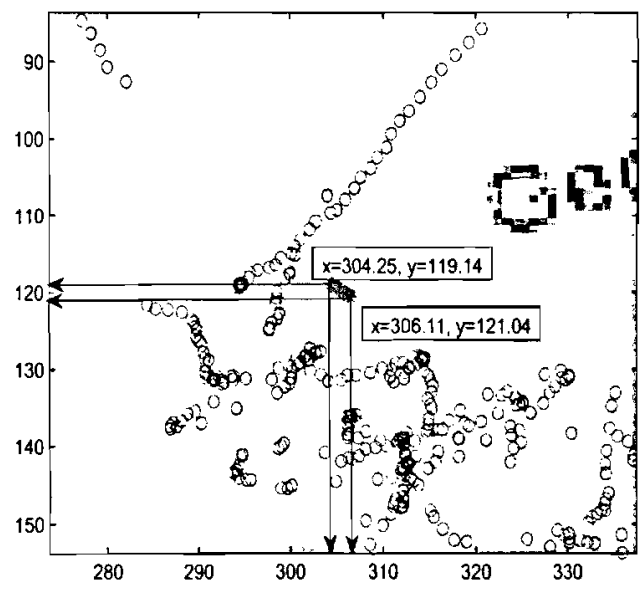

(b) Zoomed Positions.

Figure 5.15: Actual Computed Positions of a Location.

5.16(b) shows the estimated positions before (darker color) and after (lighter color) filtering, 
and Fig. 5.16(c) is the zoomed version of Fig. 5.16(b). The deviation in this case is about $0.4 \mathrm{~m}$.

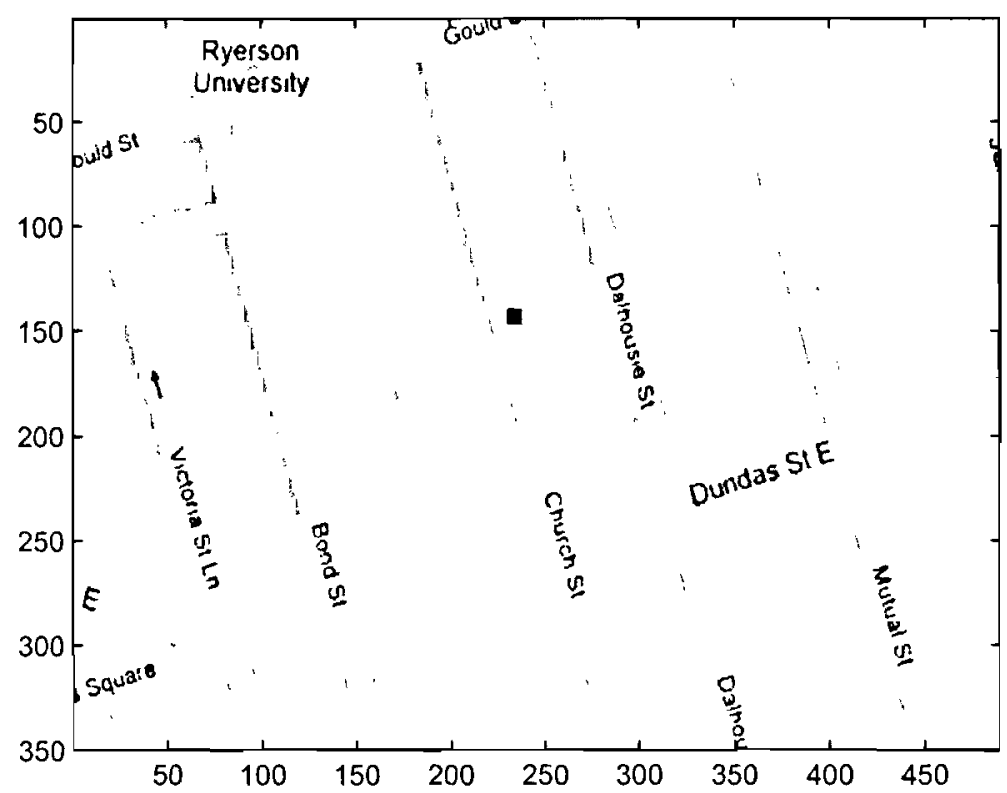

(a) True Location.

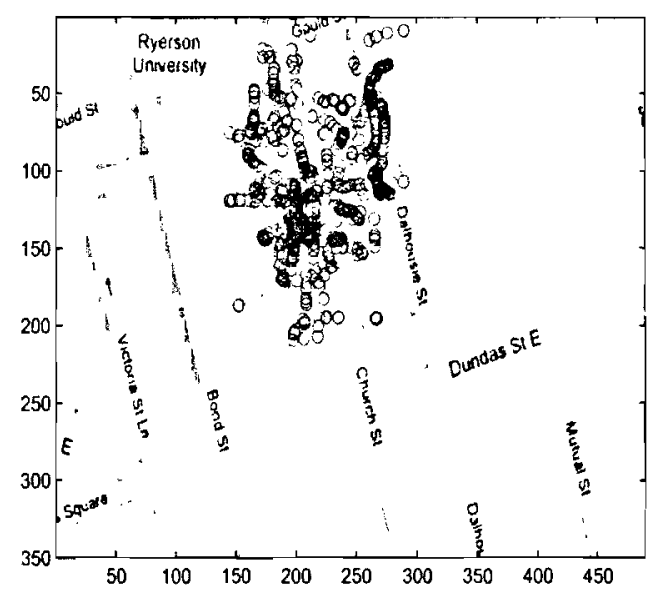

(b) Scatter Positions.

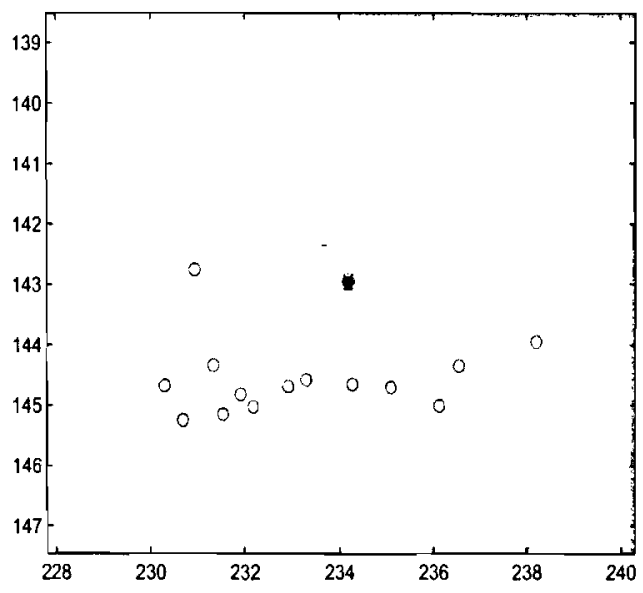

(c) Zoomed Positions.

Figure 5.16: A Location in Down Town Toronto. 


\subsection{Chapter Summary}

Fig. 5.17 provides a graphical illustration of the techniques used in this work. Basically, four or more satellites signals will be received by a receiver, and from them residuals (code minus carrier) will be made as the input to the proposed algorithm (filter 1 in Fig. 5.17). These residual will be filtered to estimate the signal distortions and subsequently their values will be subtracted from the measurements (pseudoranges) to construct the estimated ranges. These ranges will be then used to compute the positions. Although these positions show improvements compared to the unfiltered data, their standard deviation in this stage is not below $2 \mathrm{~m}$, and it is in the range of $20 \mathrm{~m}$ to $80 \mathrm{~m}$. To achieve the desired standard deviation, which is $1.5 \mathrm{~m}$ or less for about $95 \%$ of the time, the computed positions are filtered (filter 2 in Fig. 5.17) to finalize the coordinate of the user. The final positions achieved from the proposed algorithm shows with a probability of $96.5 \%$, the norm of the standard cleviation to be less than $1.5 \mathrm{~m}$. In addition to lowering the standard deviation, the proposed algorithm affected the data distribution at the end.

Although the focus of the thesis is not to gaussify the data distribution, we see that the outputs are normally distributed as shown in Fig. 5.13. Normally distributed data is a very well known feature which helps in data modeling, estimation and prediction. The achievement

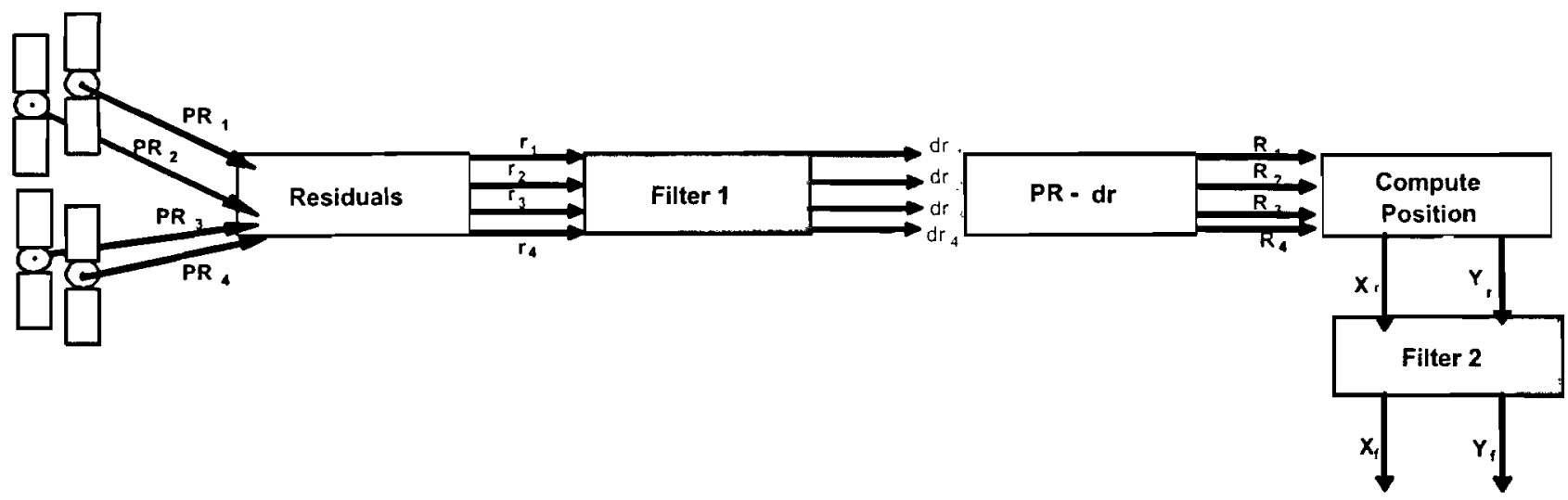

Figure 5.17: Block Diagram of the Proposed Positioning.

obtained in this work is significant. We have the outputs of an expensive receiver/antenna 
from an inexpensive (about \$500) receiver. Having a low cost GPS system with high precision opens doors to the public and industrial sectors. It is sure to offer numerous, significant and affordable solutions. We see beneficial sport/recreational applications, business solutions, child safety, and traffic control applications just to mention a few. 


\section{Chapter 6}

\section{Conclusions}

Beside hardware improvements to acquire more accuracy and precision on computed positions from a GPS receiver, different software solutions have been employed to post-process data to decrease the standard deviation of the positions. To mitigate errors which cause deviations from the true value of the calculated coordinates, wavelet analysis has been used by many researchers on data extracted from dual frequency (L1 and L2 bands) receivers, and without investigating the effect of the levels and threshold value on the positions. In all the previous works, the scholars were working with multiple high cost receivers.

In our study, we post-process the data from an inexpensive receiver based solely on L1 frequency. The proposed algorithm characterizes multipath error by employing eigenvalues and entropy of the data, and then mitigates the effect of multipath by applying multi-resolution analysis on the data. The algorithm proceeds with first, decomposing the data into different levels based on entropy. Then, two different methods of computing a threshold value are selected to filter the wavelet coefficients.

In the first method of thresholding, Donoho's estimator [24] is used, as most of the previous works in this area are based on. For the second method, the new threshold based on singular value data decomposition is proposed. The results obtained from 29 data sets collected from downtown Toronto showed significant improvement in standard deviation of the estimated positions (less than $1.5 \mathrm{~m}$ with probability of $96.5 \%$ ) when the new threshold value along with the level selection criteria applied on the data sets. 


\section{Appendix A}

\begin{tabular}{|c|c|c|c|c|}
\hline $\begin{array}{l}\text { Silcellite" } \\
\text { Il) } \\
\text { Numbet } \\
\text { Numbert }\end{array}$ & $\begin{array}{c}\text { Cil's } \\
\text { PRN } \\
\text { signal } \\
\text { Numbliner }\end{array}$ & $\begin{array}{l}\text { Conle: } \\
\text { phase } \\
\text { Soleclion } \\
\text { (C.2) }\end{array}$ & $\begin{array}{l}\text { Coula } \\
\text { 1)alas } \\
\text { ( hipis }\end{array}$ & $\begin{array}{c}\text { fint } \\
\text { lochips } \\
\text { Octal }\end{array}$ \\
\hline I & 1 & $2+1-6$ & 5 & $14+11$ \\
\hline 2 & 2 & $3+7$ & fi & 16211 \\
\hline 3 & 3 & $4+8$ & 7 & 17111 \\
\hline 4 & 4 & $5 \cdot+9$ & 8 & 1744 \\
\hline 5 & 5 & $1+9$ & 17 & 1133 \\
\hline 6 & 6 & $2+10$ & 18 & 145.5 \\
\hline 7 & 7 & $1 ! 8$ & 139 & 1131 \\
\hline 8 & 8 & $2+9$ & 140 & 14.54 \\
\hline 9 & 9 & $3 \cdot+10$ & 141 & 1626 \\
\hline (1) & 10 & $2+3$ & 251 & 15114 \\
\hline 11 & 11 & $3+4$ & 252 & 1642 \\
\hline 12 & 12 & $5 \cdot 6$ & 254 & 1750 \\
\hline 13 & 13 & $6+17$ & 23.5 & 1764 \\
\hline 14 & 14 & $7+8$ & 276 & 1772 \\
\hline 15 & 15 & $8+9$ & 257 & 1775 \\
\hline 16 & 16 & $9+10$ & 258 & 1776 \\
\hline 17 & 17 & $1+4$ & 4179 & 11.56 \\
\hline 18 & 18 & $2+5$ & 470 & 1467 \\
\hline 19 & 19 & $3+6$ & 471 & 1633 \\
\hline 20 & 20 & $4+7$ & 472 & 1715 \\
\hline 21 & 21 & $5+8$ & 473 & 1746 \\
\hline 22 & 22 & $6+9$ & 474 & 1763 \\
\hline 23 & 23 & $I-3$ & 509 & 1063 \\
\hline 24 & 24 & $41 \cdot 6$ & 512 & 1706 \\
\hline 25 & 25 & $3+7$ & 513 & 1743 \\
\hline 26 & 26 & $6+8$ & 514 & 1761 \\
\hline 27 & 27 & $7+9$ & 515 & 1770 \\
\hline 28 & 28 & $8+10$ & 516 & 1774 \\
\hline 29 & 29 & $1+6$ & 859 & 1127 \\
\hline 30 & 30 & $2+7$ & 8611 & 1453 \\
\hline 31 & 31 & $3+8$ & 861 & 1625 \\
\hline 32 & 32 & $4+9$ & 862 & 1712 \\
\hline - & 33 & $5 \cdot+10$ & 863 & 1745 \\
\hline- & 34 & $4: 10$ & 950 & 1713 \\
\hline- & 35 & $1+7$ & 947 & 1134 \\
\hline- & 36 & $2+8$ & 948 & 1456 \\
\hline- & 37 & $4+10$ & 950 & 1713 \\
\hline
\end{tabular}

Figure A.1: C/A Code Phase Assignment, taken from [2]. 


\section{Bibliography}

[1] B. Jun Yoon, P. P. Vaidyanathan, "Wavelet Based Denoising by Customized Thresholding", Vol. 2, p.p. 925-928, in Proceedings of IEEE, ICASSP (International Conference on Acoustics, Speech, and Signal Processing) 2004.

[2] B. W. Parkinson, J. J. Spilker, Global Positioning System: Theory and Applications, Vol. 1, American Institute of Aeronautics, 1996.

[3] B. Hofmann-Wellenhof, H. Lichtenegger, J. Collins, GPS Theory and Practice, Fifth Revised Edition, Springer-Verlag Wien NewYork, 2001.

[4] P. Nisra, P. Enge, "Global Positioning System: Signals, Measurements, and Performance", Ganga-Jamuna Press, 2001.

[5] A. El-Rabbany, "Advanced Satellite Positioning", Unpublished Lecture Notes, Department of Civil Engineering, Ryerson University, 2006.

[6] J. Zogg, "GPS Basics, Introduction to the System Application Overview", u-blox Corporation, www.telecom.fh-htwchur.ch/zogg/Dateien, (last accessed on: December 25, 2006).

[7] G. Xie, "Optimal On-Airport Monitoring of the Integrity of GPS-Based Landing System", A Doctor of Philosophy Dissertation, Stanford University, 2004.

[8] D. Plausinaitis, "Code Generation and Carrier Generation", Aalborg University, http://kom.aau.dk/ dpl/courses/mm11_ slides.pdf, (last accessed on: December 25, 2006). 
[9] P. H. Dana, "Global Positioning System (GPS) Overview", Kluwer Academic Publishers, Bostom, 1997.

[10] H. Kuusniemi, "User-Level Reliability and Quality Monitoring in Satellite-Based Personal Navigation", Thesis for the Degree of Doctor of Technology, Tampere University of Technology, 2005.

[11] B. P. Lathi, Modern Digital and Analog Communication Systems, Third Edition, Oxford University Press, 1998.

[12] C. Satirapod, C. Rizos, "Multipath Mitigation by Wavelet Analysis for GPS Base Station Applications", Survey Review, Vol. 38, No. 295, 2003.

[13] Y. Zhang, C. Bartone, "Real-time Multipath Mitigation with Wavesmooth Technique using Wavelets", ION GNSS 17th International Technical Meeting of the Satellite Division, pp. 1181-1193, September 2004.

[14] L. Xia, J. Liu, "Approach for Multipath Reduction Using Wavelet Algorithm", The Institute of Navigation (ION), International Technical Meeting, pp. 2131-2143, Salt Lake City, Utah, 2001.

[15] N. El-Sheimy, A. Osman, S. Nassar, A. Noureldin, "Wavelet Multiresolution Analysis", GPS World, 2003.

[16] C. S. Burrus, R. A. Gopinath, Wavelet and Wavelet Transforms, Rice University, Prentice-Hall Inc. 1998.

[17] I. Daubechies, "Ten Lectures on Wavelets", CBMS-NSF Regional Conference Series, Capital City Press, 1992.

[18] J. R. Williams, K. Amaratunga, "Introduction to Wavelets in Engineering", Intelligent Engineering Systems Laboratory, Massachusetts Institute of Technology, USA, 2000. 
[19] V. B. Gundersen, "Multivariate signal Analysis-Empirical Modeling of Drilling Riser", Telemark Institute of Technology, 1998.

[20] J. Morlet, "Sampling Theory and Wave Propagation", NATO ASI Series, SpringerVerlag, Vol.I, 233-261, 1983.

[21] B. Vidakovic, P. Mueller, "Wavelet, A Tutorial Introduction", Duke University, 1991.

[22] The MathWorks, Inc. http://www.mathworks.com/access/helpdesk/help/ techdoc/matlab.shtml, (last accessed on: December 25, 2006).

[23] H. Ahmadi, "Wavelets: Principles and Applications in Signal Processing", University of Tehran, Electrical and Computer Engineering Department, Lecture Notes, 2004.

[24] D. L. Donoho, "De-noising by Soft-Thresholding", IEEE Transactions on Information Theory 41, pp. 613-627, May 1995.

[25] M. Aram, B. Li, S. Krishnan, A. Anpalagan, "Improving Position Estimates from a Stationary GNSS Receiver Using Wavelets and Clustering", in Proceedings of IEEE CCECE, 2006.

[26] M. Aram, A. El-Rabbany,, S. Krishnan, A. Anpalagan, "Single Frequency Multipath Mitigation Based On Wavelet Analysis", The Journal of Navigation, The Royal Institute of Navigation, Vol, 60, Journal and Article number NAV414, 2007.

[27] F. Abramovich, T. Sapatinas, B.W. Silverman, "Wavelet Thresholding via a Bayesian Approach", Royal Statistical Society, 1998.

[28] K. J. Balakrishnan, N. A. Touba, "Relationship Between Entropy and Test Data Compression", in Proceedings of IEEE, Vol. 26, NO. 2, pp. 386-394, 2007.

[29] M. Shiga, Y. Yokota, "An Optimal Entropy Estimator for Discrete Random Variables", in Proceedings of IEEE, International Joint Conference on Neural Networks, Vol. 2, pp. 1280-1285, 2005. 
[30] J. T. Ho, P. J. Smith, "A Quick Simulation Method for Fading Communications Channels using a Novel Eigenvalue Importance Sampling 'Technique", in Proceedings of IEEE, Vehicular Technology Conference, Vol. 1, pp. 449-453, 2002.

[31] R. Everson, S. Roberts, "Inferring the Eigenvalues of Covariance Matrices from Limited, Noisy Data", in Proceedings of IEEE Transactions on Signal Processing, Vol. 48, pp. 2083-2091, 2000. 